\title{
Polyhomogeneous solutions of nonlinear wave equations without corner conditions
}

\author{
Piotr T. Chruściel* \\ LMPT, Fédération de recherche Denis Poisson \\ Parc de Grandmont \\ F-37200 Tours, France \\ Szymon Łȩski \\ Centrum Fizyki Teoretycznej PAN, Al. Lotników 32/46, \\ 02-668 Warsaw, Poland
}

November 19, 2018

\begin{abstract}
The study of Einstein equations leads naturally to Cauchy problems with initial data on hypersurfaces which closely resemble hyperboloids in Minkowski space-time, and with initial data with polyhomogeneous asymptotics, that is, with asymptotic expansions in terms of powers of $\ln r$ and inverse powers of $r$. Such expansions also arise in the conformal method for analysing wave equations in odd space-time dimension. In recent work [11] it has been shown that for non-linear wave equations, or for wave maps, polyhomogeneous initial data lead to solutions which are also polyhomogeneous provided that an infinite hierarchy of corner conditions holds. In this paper we show that the result is true regardless of corner conditions.
\end{abstract}

\section{Contents}

1 Introduction $\quad 2$

2 Wave equations near conformal infinity 5

2.1 Semi-linear wave equations . . . . . . . . . . . . 15

2.2 Wave maps . . . . . . . . . . . . . . . . . . . 18

3 Polyhomogeneity without corner conditions $\quad \mathbf{2 0}$

3.1 Linear equations . . . . . . . . . . . . . . . . . 20

3.2 Nonlinear equations . . . . . . . . . . . . . . 27

${ }^{*}$ E-mail Piotr.Chrusciel@lmpt.univ-tours.fr, URL www.phys.univ-tours.fr/ piotr 
$\begin{array}{lr}\text { A Function spaces, auxilliary results } & 35\end{array}$

A.1 $\mathscr{C}$-spaces . . . . . . . . . . . . . . . . 35

A.2 Polyhomogeneous functions $(\mathscr{A}$-spaces $) \ldots \ldots . \ldots 36$

A.3 $\mathscr{F}$ - and $\mathscr{T}$-spaces . . . . . . . . . . . . . . . . 41

A.4 Extensions of a class of functions . . . . . . . . . . . . 43

A.5 Integral operators on $\mathscr{A}$ - and $\mathscr{C}$-spaces . . . . . . . . . . 45

A.6 Integral operators on $\mathscr{T}$ - and $\mathscr{F}$-spaces . . . . . . . . . . 47

\section{B Polyhomogeneity of solutions for a class of linear symmetric} hyperbolic systems with smooth coefficients

\section{Introduction}

The hyperboloidal Cauchy problem provides one of the methods of studying global properties of solutions of the vacuum Einstein equations. Here one prescribes Cauchy data on a spacelike hypersurface with asymptotic behavior somewhat similar to that of a hyperboloid in a Minkowski space-time. Such Cauchy problems can be used to prove non-linear stability of Minkowski space-time within a restricted class of initial data sets, see $[1,9,13]$ and references therein. Because the initial data for Einstein equations satisfy the constraint equations, one faces the need to consider polyhomogeneous initial data in generic situations [2], that is, initial data with asymptotic expansions in terms of powers of $\ln r$ and inverse powers of $r$, where $r$ is a luminosity parameter. Such initial data are too singular at the conformal boundary at infinity to be handled by the standard theory of hyperbolic equations.

As a first step towards handling that question, in this paper we study the hyperboloidal Cauchy problem for simpler nonlinear wave equations. Indeed, a closely related approach for understanding the global behavior of solutions of wave equations on Minkowski space-time is provided by the "conformal method", which proceeds as follows: Let $(\mathscr{M}, \mathfrak{g})$ be an $(n+1)$-dimensional space-time and let

$$
\tilde{\mathfrak{g}}=\Omega^{2} \mathfrak{g} .
$$

Let $\square_{h}$ denote the wave operator associated with a Lorentzian metric $h$,

$$
\square_{h} f=\frac{1}{\sqrt{\left|\operatorname{det} h_{\rho \sigma}\right|}} \partial_{\mu}\left(\sqrt{\left|\operatorname{det} h_{\alpha \beta}\right|} h^{\mu \nu} \partial_{\nu} f\right) .
$$

Recall that the scalar curvature $R=R(\mathfrak{g})$ of $\mathfrak{g}$ is related to the corresponding scalar curvature $\tilde{R}=\tilde{R}(\tilde{\mathfrak{g}})$ of $\tilde{\mathfrak{g}}$ by the formula

$$
\tilde{R} \Omega^{2}=R-2 n\left\{\frac{1}{\Omega} \square_{\mathfrak{g}} \Omega+\frac{n-3}{2} \frac{|\nabla \Omega|_{\mathfrak{g}}^{2}}{\Omega^{2}}\right\} .
$$

It then follows from (1.2) that we have the identity

$$
\square_{\mathfrak{g}}\left(\Omega^{-\frac{n-1}{2}} f\right)=\Omega^{-\frac{n+3}{2}}\left(\square_{\mathfrak{g}} f+\frac{n-1}{4 n}\left(\tilde{R} \Omega^{2}-R\right) f\right) .
$$


When $\mathfrak{g}$ is the Minkowski metric $\eta$, a non-trivial conformal factor $\Omega \sim 1 / r$ can be chosen so that $\tilde{\mathfrak{g}}$ is also the Minkowski metric, leading to

$$
\square_{\eta}\left(\Omega^{-\frac{n-1}{2}} f\right)=\Omega^{-\frac{n+3}{2}} \square_{\eta} f .
$$

Because the conformal boundary $\{\Omega=0\}$ corresponds to points which are infinitely far away for the original metric $\mathfrak{g}$, this technique allows one to reduce global-in-time existence problems to local ones; this has been exploited by various authors [3-8] for wave equations on a fixed background space-time.

On a more modest level, the identity (1.3) can be used as a starting point for the analysis of the asymptotic behavior of solutions of the scalar wave equation, as it reduces the problem to a study of the rescaled equation (1.4) near the set $\Omega=0$. There is, however, a difficulty that arises for non-linear equations in even space-dimension because of half-integer powers of $\Omega$ in (1.4). This introduces singular terms ${ }^{1}$ in the equations, so that the usual theory of hyperbolic PDEs does not apply. This problem has been studied in [11], where it was shown that solutions for a class of semi-linear wave equations, or of the wave-map equation, with smooth or polyhomogeneous initial data on hyperboloids in Minkowski space-time, will have a complete asymptotic expansion in terms of half integer powers of $\Omega \sim 1 / r$ and of powers of $\ln \Omega$, provided that an infinite hierarchy of "corner conditions" is satisfied. The object of this paper is to show that no corner conditions are necessary for polyhomogeneity of solutions of those equations. We also extend the results in [11] to general asymptotically flat space-times with smooth, or polyhomogeneous, conformal completions. We expect to be able to prove corresponding results for Einstein equations in a near future, see also [15].

Our main results, in Minkowski space-time, are the following (we refer the reader to Appendix A.2 for the definition of the spaces involved; the hyperboloids $\mathscr{H}_{\tau}$ are defined as

$$
\mathscr{H}_{\tau}=\left\{(t-\tau)^{2}-|\vec{r}|^{2}=1\right\}
$$

the coordinate $x$ in which the polyhomogeneous expansions are carried out is $1 /|\vec{r}|)$ :

THEOREM 1.1 Let $\delta=1$ in odd space dimensions, and let $\delta=1 / 2$ in even space dimensions. Consider the equation

$$
\square_{\mathfrak{g}} f=H(f)
$$

on $\mathbb{R}^{n, 1}, n \geq 2$, with smooth or polyhomogeneous initial data on a hyperboloid $\mathscr{H}_{0}:$

$$
\left.f\right|_{\mathscr{H}_{0}} \in x^{(n-1) / 2}\left(\mathscr{A}_{\{x=0\}}^{\delta} \cap L^{\infty}\right),\left.\quad \partial_{\tau} f\right|_{\mathscr{H}_{0}} \in x^{(n-1) / 2} \mathscr{A}_{\{x=0\}}^{\delta} .
$$

\footnotetext{
${ }^{1}$ It should be mentioned that for special non-linearities the singular terms do not arise. Indeed, this is the case for the equation $\square f=H(f)$, where $H$ is a polynomial containing only odd powers of $f$. Similarly no singularities arise for the wave map equation with special targets $[5,6]$. However, e.g. wave maps will have such singular terms in general.
} 
Suppose that $H(f)$ is smooth in $f$ and has a zero of order $\ell$ at $f=0$, with $\ell$ satisfying

$$
\ell \geq \begin{cases}4, & n=2, \\ 3, & n=3 \\ 2, & n \geq 4\end{cases}
$$

There exists $\tau^{*}>0$ and a solution of (1.5)-(1.6) defined on $\cup_{\tau \in\left[0, \tau_{*}\right]} \mathscr{H}_{\tau}$ such that for every $\epsilon>0$ we have

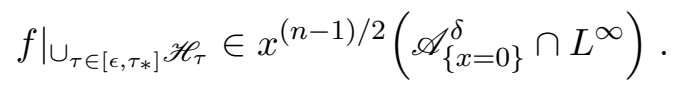

Theorem 1.1 is a special case of Theorem 2.7 below where asymptotically vacuum space-times with polyhomogeneous $C^{1}$ conformal completions at null infinity satisfying mild restrictions on the scalar curvatures are considered, with general polyhomogeneous initial data hypersurfaces, and with somewhat more general non-linearities.

Theorem 1.2 Let $\delta$ be as in Theorem 1.1. Consider the wave map equation on $\mathbb{R}^{n, 1}, n \geq 2$. For any smooth or polyhomogeneous initial data on a hyperboloid $\mathscr{H}_{0}$,

$$
\begin{gathered}
\left.x^{-(n-1) / 2} f^{a}\right|_{\mathscr{H}_{0}} \in \mathscr{A}_{\{x=0\}}^{\delta} \cap L^{\infty}, \\
\left.\partial_{\tau}\left(x^{-(n-1) / 2} f^{a}\right)\right|_{\mathscr{H}_{0}} \in \begin{cases}\mathscr{A}_{\{x=0\}}^{\delta} \cap L^{\infty}, & n=2, \\
\mathscr{A}_{\{x=0\}}^{\delta}, & n \geq 3,\end{cases}
\end{gathered}
$$

there exists $\tau^{*}>0$, and a solution $f$ defined on $\cup_{\tau \in\left[0, \tau_{*}\right]} \mathscr{H}_{\tau}$, such that for every $\epsilon>0$ we have

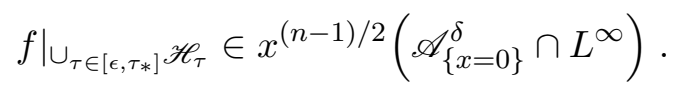

Theorem 1.2 is a special case of Theorem 2.9 below.

This paper is organised as follows. In Appendix A the reader will find a description of our notation and conventions, as well as the definitions of the function spaces used, together with some auxiliary results needed in the body of the paper. In Section 2 we show how to apply our main results, derived in later sections, to the nonlinear wave equation, or to the wave map equation, on manifolds with conformal completions at null infinity. The discussion in that section motivates the hypotheses of our polyhomogeneity theorems in Section 3, which is the cornerstone of the paper. There we prove polyhomogeneity of solutions of a class of systems of first order PDE's. In Appendix B we give a considerably simpler proof of our main polyhomogeneity theorem for linear systems, under the supplementary assumption that all coefficients in the equation are smooth and bounded.

We acknowledge useful discussions with Olivier Lengard at an early stage of this work: he suggested a proof of the result in a simple case, which was at the origin of this paper. 


\section{Wave equations near conformal infinity}

In this section we verify that those results of [11] which have been proved for the Minkowski metric can be extended to space-times with completions at null infinity. We allow conformal completions with polyhomogeneous metrics, under a condition on the scalar curvature near the conformal boundary. (In fact, metrics in weighted Sobolev spaces suffice for existence of solutions with weighted Sobolev regularity.) We also show how to write quasi-linear wave equations in a form to which our polyhomogeneity arguments in the next sections apply.

For convenience of typesetting we will denote by $g$ the metric $\tilde{\mathfrak{g}}$ from the introduction. We assume, as usual (cf., e.g., [13]), that the function $\Omega$ in (1.1) vanishes precisely at a null hypersurface which we denote by $\mathscr{N}$, with $d \Omega$ nowhere vanishing on $\mathscr{N}$. In the construction that follows we assume that $\Omega$ is either smooth or $C^{1}$ and polyhomogeneous; equivalently, $\mathscr{N}$ is a smooth or polyhomogeneous $C^{1}$ hypersurface.

Near $\mathscr{N}$ we can always find, locally, a convenient coordinate system $\left(y, x, v^{A}\right)$ so that $\mathscr{N}$ is given by the equation $x=0$, with $\partial_{y}$ tangent to the generators of $\mathscr{N}$, as follows (compare [16]; this construction can also be carried out for $\mathscr{A}_{\{x=0\}}^{\delta} \cap L^{\infty}$-polyhomogeneous metrics, see [12, Appendix B]): Let $\mathscr{O} \subset \mathscr{N}$ be any $(n-1)$-dimensional submanifold of $\mathscr{N}$, transverse to the null generators of $\mathscr{N}$. Let $v^{A}$ be any local coordinate system on $\mathscr{O}$, and let $\left.\ell\right|_{\mathscr{O}}$ be any field of null vectors, defined on $\mathscr{O}$, tangent to the generators of $\mathscr{N}$. Solving the equation $\nabla_{\ell} \ell=0$, with initial values $\left.\ell\right|_{\mathscr{O}}$ on $\mathscr{O}$, one obtains a null vector field $\ell$ defined on a $\mathscr{N}$-neighborhood $\mathscr{V} \subset \mathscr{N}$ of $\mathscr{O}$, tangent to the generators of $\mathscr{N}$. One can extend $v^{A}$ to $\mathscr{V}$ by solving the equation $\ell\left(v^{A}\right)=0$. The function $\left.y\right|_{\mathscr{N}}$ is defined by solving the equation $\ell(y)=1$ with initial value $\left.y\right|_{\mathscr{O}}=0$. Passing to a subset of $\mathscr{V}$ if necessary, this defines a global coordinate system $\left(y, v^{A}\right)$ on $\mathscr{V}$. By construction we have $\ell=\partial_{y}$ on $\mathscr{V}$, in particular $g_{y y}=0$ on $\mathscr{V}$. This justifies the first equation in (2.4) below. Further, $\ell$ is normal to $\mathscr{N}$ because $\mathscr{N}$ is a null surface, which implies $g_{y A}=0$ on $\mathscr{V}$. This justifies the second equation in (2.4) below.

Let, next, $\left.\bar{\ell}\right|_{\mathscr{V}}$ be a field of null vectors on $\mathscr{V}$ defined uniquely by the conditions

$$
g\left(\left.\bar{\ell}\right|_{\mathscr{V}}, \ell\right)=\frac{1}{2}, \quad g\left(\left.\bar{\ell}\right|_{\mathscr{V}}, \partial_{A}\right)=0
$$

The first equation implies that $\left.\bar{\ell}\right|_{\mathscr{V}}$ is everywhere transverse to $\mathscr{V}$. Then we define $\bar{\ell}$ in a space-time neighborhood $\mathscr{U} \subset \mathscr{M}$ of $\mathscr{V}$ by solving the geodesic equation $\nabla_{\bar{\ell}} \bar{\ell}=0$ with initial value $\left.\bar{\ell}\right|_{\mathscr{V}}$ at $\mathscr{V}$. The coordinates $\left(y, v^{A}\right)$ are extended to $\mathscr{U}$ by solving the equations $\bar{\ell}(y)=\bar{\ell}\left(v^{A}\right)=0$, and the coordinate $x$ is defined by solving the equation $\bar{\ell}(x)=1$, with initial value $x=0$ at $\mathscr{V}$. Passing to a subset of $\mathscr{U}$ if necessary, this defines a global coordinate system $\left(y, x, v^{A}\right)$ on $\mathscr{U}$.

By construction we have

$$
\bar{\ell}=\partial_{x},
$$

hence $\partial_{x}$ is a null, geodesic, vector field on $\mathscr{U}$. In particular

$$
g_{x x} \equiv g\left(\partial_{x}, \partial_{x}\right)=0 .
$$


Let $\left(z^{a}\right)=\left(x, v^{A}\right)$, and note that

$$
\begin{aligned}
\bar{\ell}\left(g\left(\bar{\ell}, \partial_{a}\right)\right) & =g\left(\bar{\ell}, \nabla_{\bar{\ell}} \partial_{a}\right)=g\left(\bar{\ell}, \nabla_{\partial_{x}} \partial_{a}\right)=g\left(\bar{\ell}, \nabla_{\partial_{a}} \partial_{x}\right) \\
& =g\left(\bar{\ell}, \nabla_{\partial_{a}} \bar{\ell}\right)=\frac{1}{2} \partial_{a}(g(\bar{\ell}, \bar{\ell}))=0 .
\end{aligned}
$$

This shows that the components $g_{x a}$ of the metric are $x$-independent. On $\mathscr{O}$ we have $g_{x y}=1 / 2$ and $g_{x A}=0$ by (2.1), leading to the following form of the metric

$$
g=d x \stackrel{\otimes}{d} d y+\chi d y \stackrel{\otimes}{d} d y+2 \gamma \stackrel{\otimes}{d} d y+\mu,
$$

with

$$
\left.\chi\right|_{x=0}=0,\left.\quad \gamma\right|_{x=0}=0 .
$$

The symbol $\stackrel{\otimes}{\otimes}$ denotes a symmetrized tensor product:

$$
\alpha \dot{\otimes} \gamma=\frac{1}{2}(\alpha \otimes \gamma+\gamma \otimes \alpha) .
$$

Furthermore, $\gamma=\gamma_{A} d v^{A}$ is a one-form field, and $\mu=\mu_{A B} d v^{A} \dot{\otimes} d v^{B}$ is a symmetric tensor field. By inspection of (2.3), the tensor field induced from $g$ on the surfaces $y=$ const has signature $(0,+, \ldots,+)$, thus these are null hypersurface.

An example of the coordinate system above is obtained by taking $\mathscr{N}$ to be the light-cone of the origin in $(n+1)$-dimensional Minkowski space-time, with $x=r-t, y=t+r$, then the Minkowski metric $\eta$ takes the form

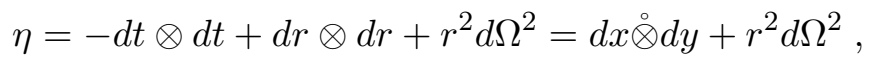

so that $\chi \equiv \gamma \equiv 0$.

In asymptotically flat solutions of asymptotically vacuum Einstein equations, a slight variation of the construction above leads to the Bondi coordinates near Scri [17], with the metric taking instead the form

$$
\tilde{g}_{B}=e^{2 \beta} d x \stackrel{\otimes}{d} d y+\chi d y \dot{\otimes} d y+2 \gamma \dot{\otimes} d y+\mu,
$$

for some function $\beta$ (and, in general, different $\chi$ and $\gamma$ ). (Here $y$ corresponds to the Bondi retarded time $u$, and $x=1 / 2 r$ is half the inverse of the luminosity distance $r$.) In $3+1$ dimensions, for smoothly compactifiable metrics, the Einstein equations imply, for matter fields decaying sufficiently fast, that $\beta=$ $O\left(x^{2}\right)$ as well as

$$
\chi=O\left(x^{2}\right), \quad \gamma_{A}=O\left(x^{2}\right),
$$

with derivatives behaving in the obvious way. One can go from (2.5) to (2.3) by redefining $x$, without changing the remaining variables, in a way which will preserve (2.6). Equation (2.6) remains valid for asymptotically vacuum metrics which, after conformal rescaling, are polyhomogeneous and $C^{1}$ (see [12, Section 6] or [10, Appendix C.1.2]), while for general $\mathscr{A}_{\{x=0\}}^{\delta} \cap L^{\infty}$-polyhomogeneous asymptotically vacuum metrics one has [12, Equations (2.15)-(2.19) with $H=$ $\left.X^{a}=0\right]$ the asymptotic behaviors $\beta=O\left(x^{2} \ln ^{N} x\right)$ and

$$
\chi=O\left(x^{2}\right), \quad \gamma_{A}=O\left(x^{2} \ln ^{N} x\right),
$$


for some $N$. Here "asymptotically vacuum" requires, for polyhomogeneous metrics, that the components of the energy-momentum tensor in asymptotically Minkowskian coordinates satisfy (see [12, end of Section 2])

$$
T_{\mu \nu}=o\left(r^{-2}\right) .
$$

Returning to (2.3), we have

$$
\operatorname{det} g=-\frac{1}{4} \operatorname{det} \mu
$$

which shows that $\mu$ is a non-degenerate $(n-1) \times(n-1)$ tensor field. It is simple to check that the inverse metric $g^{\sharp}=g^{\alpha \gamma} \partial_{\alpha} \otimes \partial_{\gamma}$ is given by the formula

$$
\begin{aligned}
g^{\sharp} & =4\left(-\chi+|\gamma|_{\mu}^{2}\right) \partial_{x} \dot{\otimes} \partial_{x}+4 \partial_{x} \dot{\otimes} \partial_{y}-4 \gamma^{\sharp} \dot{\otimes} \partial_{x}+\mu^{\sharp} \\
& =4 \partial_{x} \dot{\otimes}\left(\partial_{y}+\left(-\chi+|\gamma|_{\mu}^{2}\right) \partial_{x}-\gamma^{\sharp}\right)+\mu^{\sharp},
\end{aligned}
$$

with $\mu^{\sharp}=\mu^{A B} \partial_{A} \otimes \partial_{B}$, where $\mu^{A B}$ is the matrix inverse to $\mu_{A B}, \gamma^{\sharp}=\mu^{A B} \gamma_{A} \partial_{B}$, and $|\gamma|_{\mu}^{2}=\mu^{\sharp}(\gamma, \gamma)=\mu^{A B} \gamma_{A} \gamma_{B}$. We note

$$
g(\nabla y, \nabla y)=g^{y y}=0,
$$

which confirms the null character of the level sets of $y$, and can also be used for an alternative justification of the fact that the integral curves of

$$
\nabla y=g^{\alpha \gamma} \partial_{\alpha} y \partial_{\gamma}=g^{y \gamma} \partial_{\gamma}=2 \partial_{x}
$$

are null geodesics.

Consider a system of $N$ second order PDE's for an $m$-components field $u$, with diagonal principal part $\left(g^{\alpha \gamma} \otimes \mathrm{Id}\right) \partial_{\alpha} \partial_{\gamma}$, where Id is the $m \times m$ identity matrix:

$$
g^{\alpha \gamma} \partial_{\alpha} \partial_{\gamma} u=F\left(x^{\mu}, u, \partial u\right) .
$$

(Recall that the metric $g$ here should be thought of as the metric $\tilde{\mathfrak{g}}$ of the introduction, with the field $u$ being an appropriately rescaled equivalent of some field defined on the original space-time.) We start by checking whether such a system can be written, locally, in the form considered in Section 3 below. For this we let $e_{A}=e_{A}{ }^{C} \partial_{C}, A=1, \ldots, n-1$ be a local $\mathrm{ON}$ basis for $\mu$, and set

$$
\begin{gathered}
e_{-}=2\left(\partial_{y}+\left(-\chi+|\gamma|_{\mu}^{2}\right) \partial_{x}-\gamma^{\sharp}\right), \quad e_{+}=-2 \partial_{x}, \\
\psi_{0}=u, \quad \psi_{A}=\varphi_{A}=e_{A}(u), \quad \varphi_{+}=e_{+}(u), \quad \psi_{-}=e_{-}(u), \\
\psi=\left(\psi_{0}, \psi_{-}, \psi_{A}\right), \quad \varphi=\left(\varphi_{+}, \varphi_{A}\right) .
\end{gathered}
$$

(Factors of two and signs in (2.11) have been introduced for consistency of notation with [11].) Then

$$
\begin{aligned}
g^{\mu \nu} \partial_{\mu} \partial_{\nu} u & =2 e_{-}\left(\partial_{x} u\right)+\sum_{A}\left(e_{A}\left(e_{A}(u)\right)-e_{A}\left(e_{A}^{C}\right) \partial_{C} u\right) \\
& =2 \partial_{x}\left(e_{-}(u)\right)+2\left[e_{-}, \partial_{x}\right] u+\sum_{A}\left(e_{A}\left(e_{A}(u)\right)-e_{A}\left(e_{A}^{C}\right) f_{C}^{B} e_{B} u\right)
\end{aligned}
$$


where $f_{C}{ }^{B}$ denotes the matrix inverse to $e_{A}{ }^{B}$, and (2.10) implies the following system of equations for $(\psi, \varphi)$ :

$$
\begin{aligned}
\partial_{x} \psi_{0}+\frac{1}{2} \varphi_{+} & =0 \\
\partial_{x} \psi_{-}-\left[\partial_{x}, e_{-}\right] u= & -\frac{1}{2} \sum_{A}\left(e_{A} \varphi_{A}-e_{A}\left(e_{A}^{C}\right) f_{C}{ }^{B} \psi_{B}\right)+\frac{1}{2} F\left(x^{\mu}, \psi, \varphi\right), \\
\partial_{x} \psi_{A}-\left[\partial_{x}, e_{A}\right] u= & -\frac{1}{2} e_{A} \varphi_{+} \\
\partial_{y} \varphi_{+} & -\left(\left(-\chi+|\gamma|_{\mu}^{2}\right) \partial_{x}-\gamma^{\sharp}\right) \varphi_{+} \\
& +\frac{1}{2} \sum_{A}\left(e_{A} \psi_{A}-e_{A}\left(e_{A}{ }^{C}\right) f_{C}{ }^{B} \psi_{B}\right)-\frac{1}{2} F\left(x^{\mu}, \psi, \varphi\right), \\
\partial_{y} \varphi_{A}-\frac{1}{2}\left[e_{-}, e_{A}\right] u= & -\left(\left(-\chi+|\gamma|_{\mu}^{2}\right) \partial_{x}-\gamma^{\sharp}\right) \varphi_{A}+\frac{1}{2} e_{A} \psi_{-} .
\end{aligned}
$$

It is understood that the commutator terms have to be expressed as linear functions of $\varphi$ and $\psi$, and that those substitutions have also been done when writing $F\left(x^{\mu}, u, \partial u\right)$ as $F\left(x^{\mu}, \psi, \varphi\right)$. It follows that all the requirements concerning the principal part of (2.10), set forth in Section 3 will be satisfied for polyhomogeneous, or smooth, metrics if there exists $m$ such that

$$
\chi=O\left(x^{1+\delta} \ln ^{m} x\right), \quad \gamma_{A}=O\left(x^{1+\delta} \ln ^{m} x\right) .
$$

In situations of main physical interest with $\mathscr{N}=\mathscr{I}^{+}$the dimension is $3+1$, the space-times are asymptotically vacuum, the appropriate choice of $\delta$ is one, and Equation (2.7) shows that (2.15) holds.

In situations where (2.15) does not hold one can proceed as follows. Suppose, first, that the metric $g$ is smooth. One can then introduce new coordinates $\left(\hat{x}, \hat{y}, \hat{v}^{A}\right)$ by setting $\hat{y}=y$, and letting $\left(\hat{x}, \hat{v}^{A}\right)$ be defined as solutions of the equations

$$
e_{-}\left(\hat{v}^{A}\right)=e_{-}(\hat{x})=0,
$$

with initial values $\left.\hat{v}^{A}\right|_{y=0}=v^{A},\left.\hat{x}\right|_{y=0}=x$. In the new coordinate system we have $e_{-}=\partial_{\hat{y}}$; equivalently

$$
\frac{\partial y}{\partial \hat{y}}=1, \quad \frac{\partial x}{\partial \hat{y}}=-\chi+|\gamma|_{\mu}^{2}, \quad \frac{\partial v^{A}}{\partial \hat{y}}=-\mu^{A B} \gamma_{B} .
$$

This gets rid of all derivatives of $\varphi$ in the right-hand-sides of the last two lines of (2.14), when those equations are rewritten in terms of the hatted coordinates; e.g., the last equation in (2.14) becomes

$$
\partial_{\hat{y}} \varphi_{A}-\frac{1}{2}\left[e_{-}, e_{A}\right] u=\frac{1}{2} e_{A} \psi_{-} .
$$

(Note that we do not change the definitions (2.11)-(2.13), which still use the coordinates $\left(x, y, v^{A}\right)$ constructed above.) One can check that the remaining derivatives in (2.14) will change in a way compatible with our hypotheses in Section 3. In any case this follows from the analysis of $C_{1} \cap \mathscr{A}_{\{x=0\}}^{\delta}$ metrics, to which we pass now: We need (2.17) to be satisfied to first order of $x$ :

$$
\frac{\partial x}{\partial \hat{y}}=-\chi+|\gamma|_{\mu}^{2}+O\left(x^{1+\delta} \ln ^{m} x\right), \quad \frac{\partial v^{A}}{\partial \hat{y}}=-\mu^{A B} \gamma_{B}+O\left(x^{1+\delta} \ln ^{m} x\right)
$$


We have

$$
-\chi+|\gamma|_{\mu}^{2}, \mu^{A B} \gamma_{B} \in x C_{\infty} \cap x^{1+\delta} \mathscr{A}_{\{x=0\}}^{\delta},
$$

therefore there exist $\Upsilon, \Gamma^{A}$, smooth functions of $\left(v^{A}, y\right)$, such that

$$
\begin{aligned}
& \left(-\chi+|\gamma|_{\mu}^{2}\right)\left(x, v^{A}, y\right)=\Upsilon\left(v^{A}, y\right) x+O\left(x^{1+\delta} \ln ^{m} x\right), \\
& -\left(\mu^{A B} \gamma_{B}\right)\left(x, v^{A}, y\right)=\Gamma^{A}\left(v^{A}, y\right) x+O\left(x^{1+\delta} \ln ^{m} x\right) .
\end{aligned}
$$

We make the ansatz

$$
\hat{x}=x+x \xi\left(v^{A}, y\right), \quad \hat{v}^{A}=v^{A}+x \eta^{A}\left(v^{B}, y\right), \quad \hat{y}=y,
$$

and (2.18) leads to the following equations for $\xi, \eta^{A}$ :

$$
\Upsilon(1+\xi)+\frac{\partial \xi}{\partial y}=0, \quad \Gamma^{A}+\frac{\partial \eta^{A}}{\partial y}+\eta^{A} \Upsilon=0
$$

We impose $\xi\left(v^{A}, 0\right)=0=\eta^{A}\left(v^{B}, 0\right)$. Conversely, smooth solutions to $(2.20)$ with those initial values give (at least for $x, y$ close to 0 )

$$
\frac{\partial x}{\partial \hat{y}}=\Upsilon\left(v^{A}, y\right) x+O\left(x^{2}\right), \quad \frac{\partial v^{A}}{\partial \hat{y}}=\Gamma^{A}\left(v^{A}, y\right) x+O\left(x^{2}\right),
$$

and we end up with the following transformation rules for the derivatives:

$$
\begin{aligned}
\partial_{x} & =(1+\xi) \partial_{\hat{x}}+\eta^{A} \partial_{\hat{v}^{A}}, \\
\partial_{v^{A}} & =\partial_{\hat{v}^{A}}+x \eta^{B}{ }_{, A} \partial_{\hat{v}^{B}}+x \xi_{, A} \partial_{\hat{x}}, \\
\frac{1}{2} e_{-} & =\partial_{\hat{y}}+O\left(x^{1+\delta} \ln ^{m} x\right) .
\end{aligned}
$$

This shows that, in the hatted coordinates, for all $\mathscr{A}_{\{x=0\}}^{\delta}$-polyhomogeneous metrics, (2.14) takes a form which is compatible with our hypotheses in Section 3 .

We consider now a scalar field $u$ satisfying a non-linear massless wave equation in the physical space-time. What has been said shows that the secondderivative terms in the equation satisfy the requirements of Section 3. The zero order terms arising from the supplementary curvature term in (1.3) will be compatible with the requirements of Theorem 3.1 or Theorem 3.7 if, e.g.,

$\Omega^{-\frac{n+3}{2}}\left(R(\tilde{\mathfrak{g}}) \Omega^{2}-R(\mathfrak{g})\right) \in \mathscr{A}_{\{0 \leq x \leq y\}}^{\delta} \quad \Longleftrightarrow \quad R(\tilde{\mathfrak{g}}) \Omega^{2}-R(\mathfrak{g}) \in x^{\frac{n+3}{2}} \mathscr{A}_{\{0 \leq x \leq y\}}^{\delta}$.

One can always achieve $R(\tilde{\mathfrak{g}})=0$ by a smooth redefinition of the conformal factor $\Omega$, when $\tilde{\mathfrak{g}}$ is smooth, by solving the Yamabe equation. Similarly if the metric is $C_{1} \cap \mathscr{A}_{\{x=0\}}^{\delta}$ and $R(\tilde{\mathfrak{g}})$ satisfies

$$
R(\tilde{\mathfrak{g}}) \in \mathscr{A}_{\{x=0\}}^{\delta}
$$

(in fact $R(\tilde{\mathfrak{g}}) \in \mathscr{A}_{\{0 \leq x \leq y\}}^{\delta}$ suffices), then our results below show that this redefinition can again be made, leading to a new metric of $\mathscr{A}_{\{0 \leq x \leq y\}}^{\delta}$-differentiability 
class. $^{2}$ Then (2.21) becomes a restriction on the rate of fall-off of scalar curvature of the space-time metric $\mathfrak{g}$ near null conformal infinity $\mathscr{I}^{+}=\mathscr{N}$, in particular (2.21) will be satisfied when $\mathfrak{g}$ is vacuum. Note also that (2.7) is preserved by any conformal transformation with bounded conformal factor, so that such a redefinition of $\tilde{\mathfrak{g}}$ will not affect that equation.

Next, still for a scalar field, in order to find the first order terms which arise from $\square_{\mathfrak{g}}$ we write

$$
\begin{aligned}
\square_{g} u= & \frac{1}{\sqrt{|\operatorname{det} g|}} \partial_{\alpha}\left(\sqrt{|\operatorname{det} g|} g^{\alpha \gamma} \partial_{\gamma} u\right) \\
= & \frac{1}{\sqrt{|\operatorname{det} \mu|}}\left(2 \partial_{y}\left(\sqrt{|\operatorname{det} \mu|} \partial_{x} u\right)-2 \partial_{A}\left(\sqrt{|\operatorname{det} \mu|} \mu^{A B} \gamma_{B} \partial_{x} u\right)\right. \\
& +2 \partial_{x}\left(\sqrt{|\operatorname{det} \mu|}\left(\partial_{y} u+2\left(-\chi+|\gamma|_{\mu}^{2}\right) \partial_{x} u-\gamma^{\sharp} u\right)\right)+\Delta_{\mu} u .
\end{aligned}
$$

For $C_{0} \cap \mathscr{A}_{\{x=0\}}^{\delta}$-polyhomogeneous metrics it only remains to check whether the terms multiplying $\varphi$, as defined in (2.13), are in $C_{0} \cap \mathscr{A}_{\{x=0\}}^{\delta}$. Since all occurrences of a single $e_{A}$-derivative of $u$ can be put into $\psi_{A}$, one only needs to keep track of terms which arise as coefficients of $\varphi_{+}=e_{+}(u)=-2 \partial_{x} u$; the relevant collected contribution to $\square_{g}$ reads

$2\left(\partial_{y}(\sqrt{|\operatorname{det} \mu|})-\partial_{A}\left(\sqrt{|\operatorname{det} \mu|} \mu^{A B} \gamma_{B}\right)+2 \partial_{x}\left(\sqrt{|\operatorname{det} \mu|}\left(-\chi+|\gamma|_{\mu}^{2}\right)\right)\right) \frac{\partial_{x} u}{\sqrt{|\operatorname{det} \mu|}}$.

The coefficient of $\partial_{x} u$ will be in $C_{\infty}+x^{\delta} \mathscr{A}_{\{0 \leq x \leq y\}}^{\delta}$ if, e.g., $g \in \mathscr{A}_{\{x=0\}}^{\delta} \cap C^{1}(\widetilde{\mathscr{M}})$, where $\widetilde{\mathscr{M}}$ denotes the space-time manifold with the conformal boundary $\mathscr{I}^{+}$ added.

Summarising, we have proved:

Proposition 2.1 Consider a space-time $(\mathscr{M}, \mathfrak{g})$ with $\mathscr{A}_{\{x=0\}}^{\delta} \cap C^{1}(\widetilde{\mathscr{M}})$-polyhomogeneous conformal completion at null infinity, and suppose that (2.21) holds. The scalar wave equation on $(\mathscr{M}, \mathfrak{g})$ implies a system of equations to which Theorem 3.1 applies.

Similarly if $u$ is a solution of the non-linear wave equation (2.41) as in Theorem 1.1, or of the wave-map equation as in Theorem 1.2, then the lower order terms discussed so far are compatible with the requirements of Theorem 3.7.

We show, next, that any system of the form (2.10) can be written in a first order form as in Section 3 of [11]. Recall that in that last reference a coordinate system $\left(x, v^{A}, \tau\right)$ was used, with $\tau$ playing the role of a time variable. The solutions there were defined on the set

$$
\Omega_{x_{1}, T}=\left\{\left(x, v^{A}, \tau\right): 0<x<x_{1}-2 \tau, v \in \mathscr{O}, 0<\tau<T\right\} .
$$

\footnotetext{
${ }^{2}$ A sufficient condition for $R(\tilde{\mathfrak{g}}) \in \mathscr{A}_{\{0 \leq x \leq y\}}^{\delta}$ is that $\tilde{\mathfrak{g}}$ is of $\left(C_{\infty}+x^{2} \mathscr{A}_{\{x=0\}}^{\delta}\right)$-differentiability class, but this restriction is certainly not necessary; note that for $\delta=1$ we have $C_{\infty}+$ $x^{2} \mathscr{A}_{\{x=0\}}^{\delta}=C_{1} \cap \mathscr{A}_{\{x=0\}}^{\delta}$. It is likely that the condition $g \in C_{1} \cap \mathscr{A}_{\{x=0\}}^{\delta}$ suffices for polyhomogeneity of solutions for all $\delta$, see Remark 3.10 below.
} 


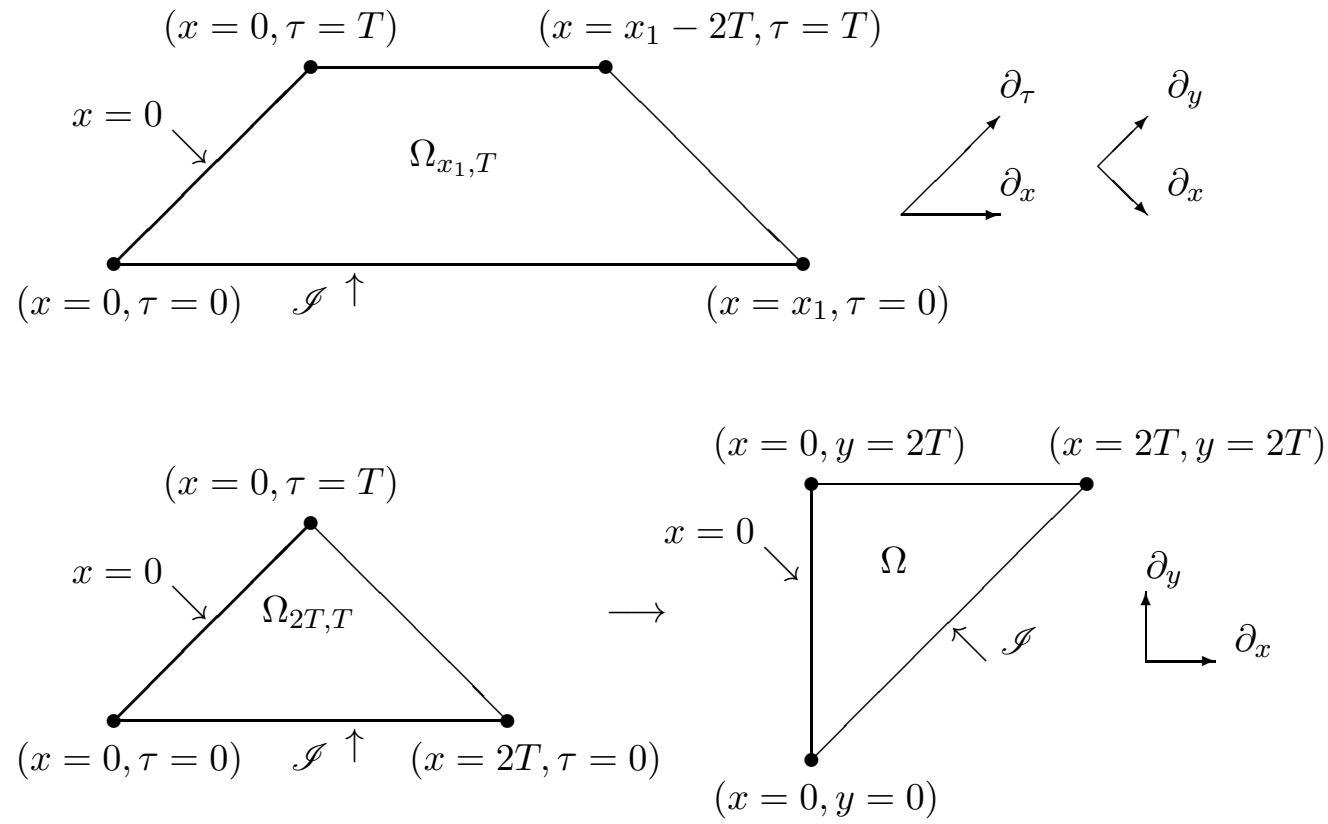

Figure 1: The variables $(x, \tau)$ and $(x, y)$.

This will be made compatible with (2.14) by replacing the coordinate $y$ with a coordinate $\tau$,

$$
(x, y) \longrightarrow\left(x, \tau=\frac{y-x}{2}\right),
$$

so that

$$
\partial_{x} \longrightarrow \partial_{x}-\frac{1}{2} \partial_{\tau}, \quad \partial_{y}=\frac{1}{2} \partial_{\tau}
$$

(see Figure 1). In this work we are interested in the behavior of solutions near the set $\{x=0\}$. For this purpose it suffices to study the properties of the solution $u$ on the following subset of $\Omega_{x_{1}, T}$ :

$$
\Omega=\left\{\left(x, v^{A}, y\right): 0<x<y, v \in \mathscr{O}, 0<y<2 T\right\} .
$$

In view of $(2.24),(2.11)$ takes the form

$$
e_{-}=\partial_{\tau}+\left(-\chi+|\gamma|_{\mu}^{2}\right)\left(2 \partial_{x}-\partial_{\tau}\right)-2 \gamma^{\sharp}, \quad e_{+}=\partial_{\tau}-2 \partial_{x} .
$$

We keep the definitions (2.12)-(2.13), and rewrite (2.14) as

$$
\begin{aligned}
& \left.\begin{array}{llll}
\left(\partial_{\tau}-2 \partial_{x}\right) \psi_{A} & -e_{A} \varphi_{+} & -\left[\partial_{\tau}-2 \partial_{x}, e_{A}\right] u & =0 \\
-\sum_{A} e_{A} \psi_{A} & +e_{-} \varphi_{+} & +\sum_{A} e_{A}\left(e_{A}{ }^{C}\right) f_{C}{ }^{B} \psi_{B} & =-F\left(x^{\mu}, \psi, \varphi\right),
\end{array}\right\} \\
& \left(\partial_{\tau}-2 \partial_{x}\right) \psi_{0}-\varphi_{+}=0 \\
& \left.\begin{array}{llll}
\left(\partial_{\tau}-2 \partial_{x}\right) \psi_{-} & -\sum_{A} e_{A} \varphi_{A} & -\left[\partial_{\tau}-2 \partial_{x}, e_{-}\right] u+\sum_{A} e_{A}\left(e_{A}^{C}\right) f_{C}^{B} \psi_{B} & =-F\left(x^{\mu}, \psi, \varphi\right), \\
-e_{A} \psi_{-} & +e_{-} \varphi_{A} & -\left[e_{-}, e_{A}\right] u & =0 .
\end{array}\right\}
\end{aligned}
$$


If we assume that the metric coefficients are $\left(\mathscr{A}_{\{x=0\}}^{\delta} \cap C_{1}\right)$-polyhomogeneous, then all coefficients in (2.27)-(2.29) are of at least $\mathscr{A}_{\{x=0\}}^{\delta} \cap C_{0}$-differentiability class.

The system (2.27)-(2.29) is of the form considered in [11, Section 3]: For example, the matrices $E_{ \pm}^{\mu}$ of Equation 3.5 of [11] take the form $E_{ \pm}^{\mu}=e_{ \pm}^{\mu} \otimes \mathrm{Id}$, with $e_{ \pm}=e_{ \pm}^{\mu} \partial_{\mu}$ as in (2.26). The covector field $n_{\mu}$ in [11, Equation (3.12)] can be taken to be equal to $d \tau$. The matrices $\ell^{A}$ can be read-off from (2.27)-(2.29):

$$
\ell^{A} \partial_{A} \psi=\left(\begin{array}{ccccc}
0 & 0 & -e_{1} & \ldots & -e_{n-1} \\
0 & -e_{1} & 0 & & 0 \\
& \vdots & & \ddots & \\
0 & -e_{n-1} & 0 & & 0
\end{array}\right)\left(\begin{array}{c}
\psi_{0} \\
\psi_{-} \\
\psi_{1} \\
\vdots \\
\psi_{n-1}
\end{array}\right)
$$

A natural choice for $L$ is then

$L \psi=\left(\begin{array}{ccccc}0 & 0 & -e_{1}-\frac{e_{1}(\sqrt{\operatorname{det} \mu})}{\sqrt{\operatorname{det} \mu}} & \ldots & -e_{n-1}-\frac{e_{n-1}(\sqrt{\operatorname{det} \mu})}{\sqrt{\operatorname{det} \mu}} \\ 0 & -e_{1} & 0 & \ddots & 0 \\ \vdots & -e_{n-1} & 0 & & 0\end{array}\right)\left(\begin{array}{c}\psi_{0} \\ \psi_{-} \\ \psi_{1} \\ \vdots \\ \psi_{n-1}\end{array}\right)$

so that

$$
\ell \psi=\left(\begin{array}{ccccc}
0 & 0 & -\frac{e_{1}(\sqrt{\operatorname{det} \mu})}{\sqrt{\operatorname{det} \mu}} & \ldots & -\frac{e_{n-1}(\sqrt{\operatorname{det} \mu}}{\sqrt{\operatorname{det} \mu}} \\
0 & 0 & 0 & & 0 \\
& \vdots & & \ddots & \\
0 & 0 & 0 & & 0
\end{array}\right)\left(\begin{array}{c}
\psi_{0} \\
\psi_{-} \\
\psi_{1} \\
\vdots \\
\psi_{n-1}
\end{array}\right) .
$$

This implies

$$
-L^{\dagger} \varphi=\left(\begin{array}{cccc}
0 & 0 & & 0 \\
0 & -\underline{\operatorname{div}} e_{1}-e_{1} & \ldots & -\underline{\operatorname{div}} e_{n-1}-e_{n-1} \\
\frac{e_{1}(\sqrt{\operatorname{det} \mu})}{\sqrt{\operatorname{det} \mu}}-\underline{\operatorname{div}} e_{1}-e_{1} & 0 & & 0 \\
\vdots & & \ddots & \\
\frac{e_{n-1}(\sqrt{\operatorname{det} \mu})}{\sqrt{\operatorname{det} \mu}}-\underline{\operatorname{div}} e_{n-1}-e_{n-1} & 0 & & 0
\end{array}\right)\left(\begin{array}{c}
\varphi_{+} \\
\varphi_{1} \\
\vdots \\
\varphi_{n-1}
\end{array}\right),
$$

where $\underline{\text { div }}$ denotes the divergence with respect to the measure $\sqrt{\operatorname{det} \mu_{A B}} d v^{1} \cdots d v^{n-1}$. It immediately follows that [11, Equations (3.9) and (3.11)] hold for $\mathscr{A}_{\{x=0\}}^{\delta} \cap C_{1}$ metrics.

We have

$$
E_{+}^{\tau}=\mathrm{Id}, \quad E_{-}^{\tau}=\left(1+\chi-|\gamma|_{\mu}^{2}\right) \mathrm{Id},
$$

which shows that the first condition in [11, Equation (3.12)] holds. On the other hand, [11, Equation (3.13)] holds only for $E_{+}^{\tau}$. However, we note the following result, where the notation from [11] is used: 
Proposition 2.2 Under the remaining conditions of [11, Proposition 3.1], suppose that instead of [11, Equation (3.13)] we have

$\left\|E_{-}^{\tau}(\tau)\right\|_{\mathscr{G}_{k}^{0}\left(M_{x_{1}-2 \tau}\right)} \leq C, \quad\left\|E_{+}^{\tau}(\tau)\right\|_{\mathscr{G}_{k}^{-1}\left(M_{x_{1}-2 \tau}\right)}+\left\|\partial_{x} E_{+}^{\tau}(\tau)\right\|_{\mathscr{G}_{k-1}^{-1}\left(M_{x}\right)} \leq \zeta(x)$.

Then the conclusions of [11, Proposition 3.1] hold.

Proof: If [11, Equation (3.13)] does not hold, then the commutators in the unnumbered equation after [11, Equation (3.28)] will have supplementary terms such as, e.g.,

$$
\sum_{i=1}^{k}\left(\begin{array}{l}
i \\
k
\end{array}\right) x^{i}\left(\partial_{x}^{i} E_{ \pm}^{\tau}\right) x^{k-i} \partial_{x}^{k-i} \partial_{\tau} \chi
$$

From the evolution equations [11, Equation (3.5)] one has

$$
\begin{aligned}
& \partial_{\tau} \varphi=\left(E_{-}^{\tau}\right)^{-1}\left(-E_{-}^{x} \partial_{x} \varphi-E_{-}^{A} \partial_{A} \varphi-\left(B_{-}+B_{11}\right) \varphi-L \psi-B_{12} \psi+a\right), \\
& \partial_{\tau} \psi=\left(E_{+}^{\tau}\right)^{-1}\left(-E_{+}^{x} \partial_{x} \psi-E_{+}^{A} \partial_{A} \psi-\left(B_{+}+B_{22}\right) \psi+L^{\dagger} \varphi-B_{21} \varphi+b\right) .
\end{aligned}
$$

This is inserted in (2.31). Some care has to be taken, because the norm used requires that each $x$-derivative comes with a factor of $x$. This works well for $\varphi$, because of the requirement in [11, Equation (3.14)] that $E_{-}^{x} \in \mathscr{G}_{k}^{1}\left(M_{x_{1}-2 \tau}\right)$. On the other hand, the $x$-derivatives of $\psi$ that result in (2.31) are rewritten as

$$
\sum_{i=1}^{k}\left(\begin{array}{l}
i \\
k
\end{array}\right)\left(x^{i-1} \partial_{x}^{i-1} \partial_{x} E_{+}^{\tau}\right) x^{k-i+1} \partial_{x}^{k-i}\left(\left(E_{+}^{\tau}\right)^{-1} E_{+}^{x} \partial_{x} \psi\right),
$$

and the estimates are done as in [11] starting from this formula. The remaining supplementary terms are handled in a similar way.

An identical argument gives:

Proposition 2.3 Under the remaining conditions of [11, Proposition 3.2], suppose that instead of [11, Equation (3.13)] we have

$$
\left\|E_{-}^{\tau}(\tau)\right\|_{\mathscr{G}_{k}^{0}\left(M_{x_{1}-2 \tau}\right)}+\left\|E_{+}^{\tau}(\tau)\right\|_{\mathscr{G}_{k}^{0}\left(M_{x_{1}-2 \tau}\right)}+\left\|\partial_{x} E_{+}^{\tau}(\tau)\right\|_{\mathscr{G}_{k-1}^{0}\left(M_{x}\right)} \leq C .
$$

Then the conclusions of [11, Proposition 3.2] hold.

The next question we wish to address is that of the choice of the initial data surface $\mathscr{S}$. The estimates of [11] were made under the assumption that

$$
\mathscr{S}=\{\tau=0\} .
$$

This might be overly restrictive for several purposes. The simplest generalisation is to assume that $\mathscr{S}$ coincides with a member of the family of hypersurfaces $\mathscr{S}_{s}$ defined as

$$
\mathscr{S}_{s}=\left\{\tau=s+\sigma\left(x, v^{A}\right)\right\},
$$


where $\sigma$ is a function satisfying

$$
\sigma \in \mathscr{C}_{\{x=0\}, k}^{1} .
$$

The field of conormals to the $\mathscr{S}_{s}$ 's is $d \tau-\partial_{x} \sigma d x-\partial_{A} \sigma d v^{A}$, so that these hypersurfaces will be space-like for the system considered in [11] if

$$
\left(\begin{array}{cc}
E_{-}^{\tau}-E_{-}^{x} \partial_{x} \sigma-E_{-}^{A} \partial_{A} \sigma & -\ell^{A} \partial_{A} \sigma \\
-\left(\ell^{A}\right)^{t} \partial_{A} \sigma & E_{+}^{\tau}-E_{+}^{x} \partial_{x} \sigma-E_{+}^{A} \partial_{A} \sigma
\end{array}\right) \geq \epsilon>0,
$$

for some constant $\epsilon$. We have

Proposition 2.4 Assume (2.36)-(2.38), and suppose that the coefficients in the equation remain in the original space after differentiation by $\left(x \partial_{\tau}\right)^{i}$, with $1 \leq$ $i \leq k-1$. Then the estimates of [11, Propositions 3.1 and 3.2], as generalised in Propositions 2.2 and 2.3, remain valid for solutions with initial data on $\mathscr{S}_{0}$, with the norms $\|f(t)\|$ in [11] understood as norms of functions on $\mathscr{S}_{t}$.

Proof: One integrates the divergence [11, Equation (3.22)] on the family of sets, parameterised by $s$,

$$
\left\{\sigma\left(x, v^{A}\right) \leq \tau \leq s+\sigma\left(x, v^{A}\right), 0 \leq x \leq x_{1}-2 \tau\right\} .
$$

The argument in [11] leads then to an estimate on the integrals over the $\mathscr{S}_{s}$ 's of $x$ - and $v^{A}$-derivatives of the fields. To relate those integrals to intrinsic Sobolev spaces on the $\mathscr{S}_{s}$ 's it is convenient to pass to a new coordinate system $\left(\tilde{\tau}, \tilde{x}, \tilde{v}^{A}\right)$ defined by the equations

$$
\tilde{\tau}=\tau-\sigma\left(x, v^{A}\right), \quad \tilde{x}=x, \quad \tilde{v}^{A}=v^{A} .
$$

We then have

$$
\partial_{\tilde{x}}=\partial_{x}+\partial_{x} \sigma \partial_{\tau}, \quad \partial_{\tilde{v}^{A}}=\partial_{v^{A}}+\partial_{v^{A}} \sigma \partial_{\tau}
$$

so that

$$
\begin{aligned}
& \tilde{x}^{i} \partial_{\tilde{v}}^{\gamma} \partial_{\tilde{x}}^{i} f=x^{i} \partial_{v}^{\gamma} \partial_{x}^{i} f+ \\
& \quad \sum C\left(j_{0}, \ldots, j_{k}, \gamma_{0}, \ldots, \gamma_{k}\right)\left(x^{j_{0}+k} \partial_{v}^{\gamma_{0}} \partial_{x}^{j_{0}} \partial_{\tau}^{k} f\right)\left(x^{j_{1}} \partial_{x}^{j_{1}} \partial_{v}^{\gamma_{1}} \sigma\right) \ldots\left(x^{j_{k}} \partial_{x}^{j_{k}} \partial_{v}^{\gamma_{k}} \sigma\right)
\end{aligned}
$$

where the sum is taken over all indices satisfying $j_{0}+\ldots+j_{k}=i,\left|\gamma_{0}\right|+\ldots+\left|\gamma_{k}\right|=$ $|\gamma|$, with $1 \leq k \leq i+|\gamma|$. One can use (2.32)-(2.33) to eliminate $\tau$-derivatives; the fact that such derivatives of $\psi$ are replaced by $x$-derivatives introduces the restriction $\partial_{A} \sigma=O(x)$ when $k=1$, and to (2.37) for higher $k$.

To proceed further, one needs to make a choice for the scalar products and covariant derivative operators. We assume that $u=\left(u^{a}\right)$ maps the original space-time into $\mathbb{R}^{m}$, with canonical scalar product. For a scalar field we take $m=1$. In case of wave maps, we will be interested in maps that asymptote a point $p$ in the target manifold when we approach $\{x=0\}$. We choose normal coordinates near $p$, and the $u^{a}$ 's are the coordinate components of the map. 
We take $E_{ \pm}^{\mu} \nabla_{\mu}=E_{ \pm}^{\mu} \partial_{\mu}$, so that the matrices $B_{ \pm}$in [11, Equation (3.16)] vanish. We choose

$$
\begin{aligned}
|\psi|^{2} & =\sum_{a, A}\left(\psi_{A}^{a}\right)^{2}+\sum_{a}\left(\left(\psi_{0}^{a}\right)^{2}+\left(\psi_{-}^{a}\right)^{2}\right), \\
|\varphi|^{2} & =\sum_{a, A}\left(\varphi_{A}^{a}\right)^{2}+\sum_{a}\left(\varphi_{+}^{a}\right)^{2} .
\end{aligned}
$$

The "energy-momentum vector" $X$ in [11, Equation (3.21)] equals then

$$
X=|\varphi|^{2} e_{-}+|\psi|^{2} e_{+},
$$

and we use the usual measure $\sqrt{|\operatorname{det} g|} d^{n+1} x=\frac{1}{2} \sqrt{|\operatorname{det} \mu|} d^{n+1} x$ to integrate over domains in the (conformally rescaled) space-time, so that

$$
\operatorname{div} X=\nabla_{\mu} X^{\mu}=\partial_{\mu}\left(\sqrt{|\operatorname{det} \mu|} X^{\mu}\right) / \sqrt{|\operatorname{det} \mu|} .
$$

Rather than deriving $\nabla_{\alpha} E_{ \pm}^{\alpha}$ from first principles, it is simpler to calculate directly $\operatorname{div} X$ using (2.39) and estimate the corresponding terms. This gives

$$
\left|\nabla_{\alpha} E_{ \pm}^{\alpha}\right| \leq\left|\nabla_{\alpha} e_{ \pm}^{\alpha}\right|
$$

It now follows from (2.11) that the relevant estimates in [11, Equation (3.14)] and [11, Equation (3.15)] hold for any $\left(\mathscr{A}_{\{x=0\}}^{\delta} \cap C_{1}\right)$-polyhomogeneous metric.

Summarising, we have proved:

Proposition 2.5 The estimates of [11, Propositions 3.1 and 3.2] hold for the scalar wave equation

$$
\square_{\mathfrak{g}} u=0
$$

on any space-time with $\left(\mathscr{A}_{\{x=0\}}^{\delta} \cap C_{1}\right)$-polyhomogeneous conformal completion at null infinity for which

$$
\Omega^{-\frac{n+3}{2}}\left(R(\tilde{\mathfrak{g}}) \Omega^{2}-R(\mathfrak{g})\right) \in \mathscr{A}_{\{x=0\}}^{\delta} .
$$

\subsection{Semi-linear wave equations}

We continue with existence of solutions of the semi-linear wave equation:

$$
\square_{\mathfrak{g}} f=H\left(x^{\mu}, f\right) .
$$

The spaces $\mathscr{H}_{\ell}^{\beta}\left(\mathscr{S}_{0}\right)$ below are defined in [11, Appendix A]:

THEOREM 2.6 Consider a space-time $(\mathscr{M}, \mathfrak{g})$ with $\mathscr{A}_{\{x=0\}}^{\delta} \cap C_{1}(\widetilde{\mathscr{M}})$-polyhomogeneous conformal completion at null infinity satisfying (2.40), where $\delta$ is the inverse of a strictly positive integer. Let $\mathscr{S}_{0}$ be a spacelike hypersurface in $\mathscr{M}$ of the form (2.36), with $\sigma$ satisfying (2.37) together with

$$
\liminf _{x \rightarrow 0} \partial_{x} \sigma>-1 / 2
$$


Set

$$
\tilde{f}=\Omega^{-(n-1) / 2} f,
$$

and suppose that the initial data for (2.41) on $\mathscr{S}_{0}$ satisfy, for some $k>\left\lfloor\frac{n}{2}\right\rfloor+1$ and $-1<\alpha<-1 / 2$,

$$
\begin{gathered}
\left.\widetilde{f}\right|_{\mathscr{S}_{0}} \in \mathscr{H}_{k+1}^{\alpha}\left(\mathscr{S}_{0}\right),\left.\quad \partial_{x} \widetilde{f}\right|_{\mathscr{S}_{0}} \in\left(\mathscr{H}_{k}^{\alpha-1 / 2} \cap \mathscr{C}_{\{x=0\}, 0}^{\alpha}\right)\left(\mathscr{S}_{0}\right), \\
\left.\partial_{\tau} \widetilde{f}\right|_{\mathscr{S}_{0}} \in \mathscr{H}_{k}^{\alpha}\left(\mathscr{S}_{0}\right) .
\end{gathered}
$$

Suppose that $H\left(x^{\mu}, f\right)$ is smooth in $f$ at fixed $x^{\mu}$, bounded and $\delta$-polyhomogeneous in $x^{\mu}$ at constant $f$, and has a zero of order $\ell$ at $f=0$, with $\ell$ satisfying (1.7). There exists $T>0$ and a solution of (2.41) defined on $\cup_{s \in[0, T]} \mathscr{S}_{s}$ such that

$$
\begin{gathered}
\tilde{f} \in \mathscr{C}_{\{0 \leq x \leq y\}, k-1-\left\lfloor\frac{n}{2}\right\rfloor}^{\alpha}\left(\cup_{s \in[0, T]} \mathscr{S}_{s}\right) \cap L^{\infty}, \\
\partial_{x} \tilde{f} \in \mathscr{C}_{\{0 \leq x \leq y\}, k-1-\left\lfloor\frac{n}{2}\right\rfloor}^{\alpha-1 / 2}\left(\cup_{s \in[0, T]} \mathscr{S}_{s}\right) \cap \mathscr{C}_{\{x=0\}, 0}^{\alpha}, \\
\partial_{\tau} \tilde{f} \in \mathscr{C}_{\{0 \leq x \leq y\}, k-1-\left\lfloor\frac{n}{2}\right\rfloor}^{\alpha}\left(\cup_{s \in[0, T]}^{\alpha} \mathscr{S}_{s}\right) .
\end{gathered}
$$

Proof: The proof of existence is essentially a repetition of that of [11, Theorem 4.1]. Condition (2.42) guarantees that (2.38) holds for $x$ small enough. Using the results established earlier in this section, one proceeds as in [11] until Equation [11, Equation (4.32)]. When $\tilde{\mathfrak{g}}$ is smooth, that last equation is handled by introducing the variables $\left(\hat{x}, \hat{y}, \hat{v}^{A}\right)$ as in (2.16). This leads again to the estimate [11, Equation (4.33)]. In order to obtain [11, Equation (4.34)] one can proceed as follows: in the coordinate system $\left(x, y, v^{A}\right)$ we have $\partial_{x} \widetilde{f}=-\frac{1}{2} \varphi_{+}$, and integration in $x$ until the initial data hypersurface $\left\{\tau=\sigma\left(x, v^{A}\right)\right\}$ gives

$$
\widetilde{f}\left(x, y, v^{A}\right)=\widetilde{f}\left(x_{0}\left(y, v^{A}\right), y, v^{A}\right)+\frac{1}{2} \int_{x}^{x_{0}\left(y, v^{A}\right)} \varphi_{+}\left(s, y, v^{A}\right) d s,
$$

where $x_{0}$ is implicitly defined by the equation

$$
x_{0}+2 \sigma\left(x_{0}, v^{A}\right)=y
$$

(note that (2.42) implies that the left-hand-side is a strictly monotonous function of $x_{0}$, guaranteeing solvability of (2.45)). Reverting to the variables $\left(\tau, x, v^{A}\right)$, this leads to the estimate

$$
\left|\widetilde{f}\left(\tau, x, v^{A}\right)\right| \leq\left\|\widetilde{f} \mid \mathscr{S}_{0}\right\|_{L^{\infty}}+\frac{1}{2} \sup _{s \in[0, \tau]}\left\|\varphi_{+}(s)\right\|_{\mathscr{C}_{\{x=0\}, 0}^{\alpha}} \int_{x}^{x_{1}} s^{-\alpha} d s .
$$

Clearly, $\sup _{s \in[0, \tau]}\left\|\varphi_{+}(s)\right\|_{\mathscr{C}_{\{x=0\}, 0}^{\alpha}}$ satisfies the same estimate [11, Equation (4.33)] as $\left\|\varphi_{+}(\tau)\right\|_{\mathscr{C}_{\{x=0\}, 0}^{\alpha}}$, which allows us to recover [11, Equation (4.34)]. The remaining arguments of [11, Theorem 4.1] apply without changes. This provides existence, and space-derivative estimates, for smooth metrics $\tilde{\mathfrak{g}}$.

When $\tilde{\mathfrak{g}}$ is polyhomogeneous, a little more work is needed: the variables $\left(\hat{x}, \hat{y}, \hat{v}^{A}\right)$ are introduced as in (2.19). This allows us to rewrite the fourth equation in (2.14) in the form

$$
\partial_{\hat{y}} \varphi_{+}+\underbrace{\Gamma}_{\in C_{\infty}} \varphi_{+}=\rho
$$


where, in the notation of [11], the source term can be estimated as, for any $\lambda<0$,

$$
\begin{aligned}
\|\rho(\tau)\|_{\mathscr{C}_{\{x=0\}, 0}^{\lambda}} \leq & C(\underbrace{\|\psi(\tau)\|_{\mathscr{C}_{\{x=0\}, 1}^{\lambda}}+\|G(\tau)\|_{\mathscr{C}_{\{x=0\}, 0}^{\lambda}}}_{\leq C E_{\lambda}(\tau)} \\
& +\underbrace{\|\varphi(\tau)\|_{\mathscr{C}_{\{x=0\}, 1}^{\lambda-\delta+\epsilon}}}_{\leq C\|\varphi(\tau)\|_{\mathscr{C}_{\{x=0\}, 1}^{\lambda-\frac{1}{2}+\epsilon} \leq C E_{\lambda+\epsilon}}(\tau)}+\|\tilde{f}(\tau)\|_{L^{\infty}}) .
\end{aligned}
$$

Here $G=-\Omega^{-(n+3) / 2} H$. The last term above arises from the scalar curvature terms in (1.3). The number $\epsilon>0$, due to possible powers of $\ln x$ in the coefficients in the equation, can be chosen to be arbitrarily small. We choose $\epsilon$ so that $\alpha-\epsilon>-1$, set $\lambda=\alpha-\epsilon$ and obtain an obvious modification of [11, Equation (4.33)] with $\alpha$ there replaced by $\lambda$. Equation (2.46) with $\alpha$ replaced by $\alpha-\epsilon$ leads then to the following variation of [11, Equation (4.34)]:

$\left|\widetilde{f}\left(\tau, x, v^{A}\right)\right| \leq\left\|\left.\widetilde{f}\right|_{\mathscr{S}_{0}}\right\|_{L^{\infty}}+C e^{C \tau}\left\|\varphi_{+}(0)\right\|_{\mathscr{C}_{\{x=0\}, 0}^{\alpha-\epsilon}}+\int_{0}^{\tau} e^{C(\tau-s)} C\left(E_{\alpha}(s), E_{\alpha-\epsilon}(s),\|\widetilde{f}(s)\|_{L^{\infty}}\right) d s$,

for a certain continuous function $C$, bounded on bounded sets. We combine the estimate [11, Equation (4.29)] with its equivalent where $\alpha$ is replaced by $\alpha-\epsilon$ to obtain

$$
\begin{aligned}
\|\widetilde{f}(\tau)\|_{L^{\infty}}+E_{\alpha}(\tau)+E_{\alpha-\epsilon}(\tau) \leq & \left\|\left.\widetilde{f}\right|_{\mathscr{S}_{0}}\right\|_{L^{\infty}}+C e^{C \tau}\left(E_{\alpha}(0)+E_{\alpha-\epsilon}(0)+\left\|\varphi_{+}(0)\right\|_{\mathscr{C}_{\{x=0\}, 0}^{\alpha-\epsilon}}\right) \\
& +\int_{0}^{\tau} \Phi\left(\tau, s,\|\widetilde{f}(s)\|_{\left.L^{\infty}, E_{\alpha}(s), E_{\alpha-\epsilon}(s)\right) d s,}\right.
\end{aligned}
$$

and one concludes as in [11].

The estimates (2.43) on the time-derivatives are obtained by a repetition of the argument of the case $m=0$ of [11, Theorem 4.4], together with a weighted Sobolev embedding.

We are ready now to prove the first main result of this paper:

Theorem 2.7 Let $\delta=1$ in odd space dimensions, and let $\delta=1 / 2$ in even space dimensions. Under the hypotheses of Theorem 2.6, suppose moreover that $H$ is $\mathscr{A}_{\{0 \leq x \leq y\}}^{\delta}$-polyhomogeneous in $x^{\mu}$ with a uniform zero of order $l$, and that both the function $\sigma$ defining $\mathscr{S}_{0}$, and the initial data are polyhomogenous:

$$
\begin{aligned}
& \sigma \in \mathscr{A}_{\{x=0\}}^{\delta} \cap C_{1} \\
& \left.f\right|_{\mathscr{S}_{0}} \in x^{(n-1) / 2}\left(\mathscr{A}_{\{x=0\}}^{\delta} \cap L^{\infty}\right),\left.\quad \partial_{\tau} f\right|_{\mathscr{S}_{0}} \in x^{(n-1) / 2} \mathscr{A}_{\{x=0\}}^{\delta} .
\end{aligned}
$$

Then the solution given by Theorem 2.6 satisfies

$$
f \in x^{(n-1) / 2}\left(\mathscr{A}_{\{0 \leq x \leq y\}}^{\delta} \cap L^{\infty}\right)\left(\cup_{s \in[0, T]} \mathscr{S}_{s}\right) .
$$

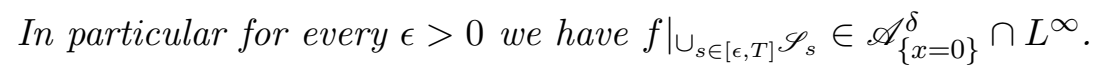


Proof: Setting $\mathscr{O}=\mathscr{S}_{0} \cap \mathscr{N}$ in the construction at the beginning of this section leads to a coordinate system in which $\mathscr{S}_{0} \cap \mathscr{N} \subset\{y=0\}$. As explained at the beginning of Section 3.1, we can then without loss of generality assume that $\mathscr{S}_{0}=\{y=x\}$. Theorem 2.6 with $k=\infty$ and Proposition 2.1 show that the hypotheses of Theorem 3.7 below are satisfied, and the result follows.

More precise information on the behavior of $\tilde{f}$ can be obtained from Theorem 3.7 and Remarks 3.8-3.9 below.

\section{$2.2 \quad$ Wave maps}

Let $(N, h)$ be a smooth Riemannian manifold, and let $f:(\mathscr{M}, \mathfrak{g}) \rightarrow(N, h)$ solve the wave map equation. As already pointed out, we will be interested in maps $f$ which have the property that $f$ approaches a constant map $f_{0}$ as $r$ tends to infinity along lightlike directions, $f_{0}(x)=p_{0} \in N$ for all $x \in \mathscr{M}$. For the purposes of the analysis in [11] it was useful to use normal coordinates around $p_{0}$, and if we write $f=\left(f^{a}\right), a=1, \ldots, \operatorname{dim} N$, then the functions $f^{a}$ satisfy the set of equations

$$
\square_{\mathfrak{g}} f^{a}+\mathfrak{g}^{\mu \nu} \Gamma_{b c}^{a}(f) \frac{\partial f^{b}}{\partial x^{\mu}} \frac{\partial f^{c}}{\partial x^{\nu}}=0,
$$

where the $\Gamma_{b c}^{a}$ 's are the Christoffel symbols of the metric $h$. Setting as before $\tilde{f}^{a}=\Omega^{-\frac{n-1}{2}} f^{a}, \tilde{\mathfrak{g}}=\Omega^{2} \mathfrak{g}$, we then have from (1.3),

$\square_{\tilde{\mathfrak{g}}} \widetilde{f}^{a}=-\Omega^{-\frac{n-1}{2}} \tilde{\mathfrak{g}}^{\mu \nu} \Gamma_{b c}^{a}\left(\Omega^{\frac{n-1}{2}} \widetilde{f}\right) \frac{\partial\left(\Omega^{\frac{n-1}{2}} \widetilde{f}^{b}\right)}{\partial x^{\mu}} \frac{\partial\left(\Omega^{\frac{n-1}{2}} \widetilde{f}^{c}\right)}{\partial x^{\nu}}+\frac{n-1}{4 n} \Omega^{-\frac{n+3}{2}}\left(\tilde{R}-R \Omega^{-2}\right) \widetilde{f}^{a}$.

Proceeding as in (2.11)-(2.13) with each component $f^{a}$ of $f$, we obtain the system of Equations (2.14), with the obvious replacements associated with $\widetilde{f}=$ $\Omega^{\frac{-(n-1)}{2}} f \rightarrow \widetilde{f}^{a}$, and with $F=\left(F^{a}\right)$ taking the form

$$
\begin{aligned}
F^{a}: & =-\Gamma_{b c}^{a}\left(\Omega^{\frac{n-1}{2}} \widetilde{f}\right) \Omega^{\frac{n-1}{2}}\left\{-\varphi_{+}^{b} \psi_{-}^{c}+\varphi_{A}^{b} \varphi_{A}^{c}\right. \\
& -\frac{n-1}{2 \Omega}\left(e_{+}(\Omega) \psi_{0}^{b} \psi_{-}^{c}+e_{-}(\Omega) \psi_{0}^{c} \varphi_{+}^{b}\right)+\frac{n-1}{\Omega} e_{A}(\Omega) \varphi_{A}^{b} \psi_{0}^{c} \\
& \left.+\frac{(n-1)^{2}}{4 \Omega^{2}}\left|\nabla_{\tilde{\mathfrak{g}}} \Omega\right|^{2} \psi_{0}^{b} \psi_{0}^{c}\right\}+\frac{n-1}{4 n}\left(\tilde{R}-R \Omega^{-2}\right) \psi_{0}^{a} .
\end{aligned}
$$

We have

ThEOREM 2.8 Consider a space-time $(\mathscr{M}, \mathfrak{g})$ with $\mathscr{A}_{\{x=0\}}^{\delta} \cap C_{1}(\widetilde{\mathscr{M}})$-polyhomogeneous conformal completion at null infinity satisfying (2.40), where $\delta$ is the inverse of a strictly positive integer. Suppose that the conformal factor $\Omega$ is of $\left(C_{2} \cap\right.$ $\left.\mathscr{A}_{\{x=0\}}^{\delta}\right)$-differentiability class. Let $\mathscr{S}_{0}$ be a spacelike hypersurface in $\mathscr{M}$ of the form (2.36), with $\sigma \in \mathscr{C}_{\{x=0\}, k}^{1}$ satisfying (2.42). Suppose that the initial data for (2.50) on $\mathscr{S}_{0}$ satisfy

$$
\left.\left.\tilde{f}^{a}\right|_{\mathscr{S}_{0}} \equiv \Omega^{\frac{-(n-1)}{2}} f^{a}\right|_{\mathscr{S}_{0}} \in \begin{cases}\left(\mathscr{H}_{k+1}^{\alpha} \cap L^{\infty}\right)\left(\mathscr{S}_{0}\right), & n \geq 3, \\ \left(\mathscr{H}_{k+1}^{\alpha} \cap \mathscr{C}_{1}^{0}\right)\left(\mathscr{S}_{0}\right), & n=2,\end{cases}
$$




$$
\begin{aligned}
\left.\partial_{x} \tilde{f}^{a}\right|_{\mathscr{S}_{0}} & \in \mathscr{H}_{k}^{\alpha}\left(\mathscr{S}_{0}\right), \\
\left.\partial_{\tau} \widetilde{f}^{a}\right|_{\mathscr{S}_{0}} & \in \begin{cases}\mathscr{H}_{k}^{\alpha}\left(\mathscr{S}_{0}\right), & n \geq 3, \\
\left(\mathscr{H}_{k}^{\alpha} \cap L^{\infty}\right)\left(\mathscr{S}_{0}\right), & n=2 .\end{cases}
\end{aligned}
$$

for some $k>\frac{n}{2}+1,-1<\alpha \leq-1 / 2$. If $n=2$ we will moreover assume $a$ single "corner condition"

$$
\left.\partial_{\tau}^{2} \widetilde{f}^{a}\right|_{\mathscr{S}_{0}} \in \mathscr{H}_{k-1}^{-1}\left(\mathscr{S}_{0}\right)
$$

There exists $T>0$ and a solution of (2.41) defined on $\cup_{s \in[0, T]} \mathscr{S}_{s}$ such that

$$
\begin{aligned}
& \tilde{f} \in \mathscr{C}_{\{0 \leq x \leq y\}, k-1-\left\lfloor\frac{n}{2}\right\rfloor}^{\alpha}\left(\cup_{s \in[0, T]} \mathscr{S}_{s}\right) \cap L^{\infty}, \\
& \partial_{x} \tilde{f}, \partial_{\tau} \tilde{f} \in \mathscr{C}_{\{0 \leq x \leq y\}, k-1-\left\lfloor\frac{n}{2}\right\rfloor}^{\alpha}\left(\cup_{s \in[0, T]} \mathscr{S}_{s}\right),
\end{aligned}
$$

for $n \geq 3$. If $n=2$ the differentiability indices in (2.55) have to be replaced by $k-3$.

Proof: The proof of existence is, again, a repetition of that of [11, Theorem 5.1]. The reader will note that, by definition of null infinity, the function $|\nabla \Omega|_{\tilde{\mathfrak{g}}}^{2}$ vanishes at $\mathscr{N}$ so that $|\nabla \Omega|_{\tilde{\mathfrak{g}}}^{2} / \Omega \in L^{\infty}$. Similarly $e_{-}(\Omega) / \Omega \in L^{\infty}$, which implies that all those terms in (2.50) which are already present in [11, Equation (5.3)] have the right structure. The last term in the third line of (2.50), which is new, can be absorbed in the linear part of the operator. The last term in the second line of (2.50), which again is not present in [11, Equation (5.3)], can be controlled using $a b \leq a^{2}+b^{2}$, which allows one to absord this term in the remaining ones (note that $e_{A}(\Omega) / \Omega \in L^{\infty}$ ) for the purpose of estimates. Those estimates can be done as in [11] until Equation [11, Equation (5.22)], which is replaced by (2.44). This leads to the inequality [11, Equation (5.23)] (the $\varphi_{-}$term there is actually not necessary in the intermediate inequality). In space-dimension two one further obtains [11, Equation (5.24)] by differentiating (2.44) with respect to $v^{A}$.

The estimates (2.55) on the time-derivatives are obtained by a repetition of the argument of the case $m=0$ of [11, Theorems 5.4 and 5.5].

This leads us to the second main result of this paper, which is a consequence of Theorems 2.8 and 3.7, as in the proof of Theorem 2.7:

Theorem 2.9 Let $\delta=1$ in odd space dimensions, and let $\delta=1 / 2$ in even space dimensions. Under the hypotheses of Theorem 2.8, suppose moreover that both the function $\sigma$ defining $\mathscr{S}_{0}$, and the initial data are polyhomogenous:

$$
\begin{aligned}
& \sigma \in \mathscr{A}_{\{x=0\}}^{\delta} \cap W^{1, \infty},
\end{aligned}
$$

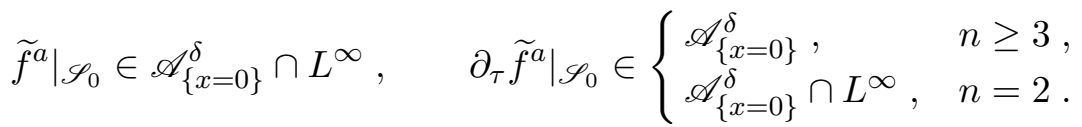

If $n=2$ we will moreover assume the corner condition

$$
\left.\partial_{\tau}^{2} \widetilde{f}^{a}\right|_{\mathscr{S}_{0}}=o\left(x^{-1}\right) .
$$


Then the solution given by Theorem 2.8 satisfies

$$
f \in x^{(n-1) / 2}\left(\mathscr{A}_{\{0 \leq x \leq y\}}^{\delta} \cap L^{\infty}\right)\left(\cup_{s \in[0, T]} \mathscr{S}_{s}\right) .
$$

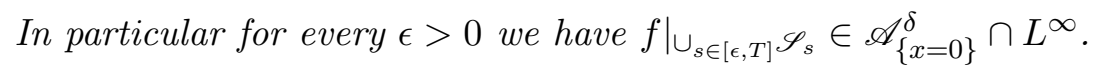

As before, the reader will find more precise information on the behavior of $\widetilde{f}$ in Theorem 3.7 and Remarks 3.8-3.9 below.

\section{Polyhomogeneity without corner conditions}

We are ready now to prove the polyhomogeneity of solutions for a class of linear systems of equations of the form

$$
\begin{aligned}
\partial_{y} \varphi+B_{\varphi \varphi} \varphi+B_{\varphi \psi} \psi & =L_{\varphi \varphi} \varphi+L_{\varphi \psi} \psi+a, \\
\partial_{x} \psi+B_{\psi \varphi} \varphi+B_{\psi \psi} \psi & =L_{\psi \varphi} \varphi+L_{\psi \psi} \psi+b,
\end{aligned}
$$

where $\varphi$ and $\psi$ are vector valued, the $B$ 's are linear maps and the $L$ 's are first order linear differential operators, while $a$ and $b$ are - possibly non-linear source terms. Such equations arise from semi- and quasi-linear wave equations in Minkowski space-time, say

$$
\square u=F(x, u, \partial u) .
$$

As discussed in detail in Section 2, one obtains a system of the form (3.1) by introducing

$$
\psi=\left(u, \partial_{y} u\right), \quad \varphi=\partial_{x} u .
$$

Then both source terms $a$ and $b$ in (3.1) are proportional to $F$. For several classes of non-linearities, solutions of such equations with polyhomogeneous (or smooth) initial data will satisfy $u \in \mathscr{C}_{\{0 \leq x \leq y\}, \infty}^{\alpha} \cap L^{\infty}$ [11], see also Section 2 . It also follows from the results there that the natural polyhomogeneity space for solutions of (3.2) is $u \in \mathscr{A}_{\{x=0\}}^{\delta}, \delta=1 / 2$ in even space-dimensions, and $\delta=1$ in odd dimensions, at least when an infinite number of corner conditions is satisfied. For such solutions $u$ and $\partial_{y} u$ will typically be bounded, so that $\psi$ will be bounded, while $\varphi$ will typically blow up, at $x=0$, as $x^{\delta-1} \ln ^{N} x$ for some $N$. The source terms $a$ and $b$ behave then in a similar way near $x=0$. Since $b$ appears in an equation where the polyhomogeneous function $\psi$ is differentiated, one expects $b$ to behave again as $x^{\delta-1} \ln ^{N^{\prime}} x$ for some $N^{\prime}$. This discussion justifies the hypotheses of our theorems below.

\subsection{Linear equations}

We consider the Cauchy problem with polyhomogeneous coefficients, polyhomogeneous sources, as well as polyhomogeneous initial data given on a polyhomogeneous hypersurface

$$
\mathscr{S}=\{y=\lambda(x, v)\}, \quad \text { with } \lambda(0, v)=0, \partial_{x} \lambda(0, v)>0,
$$


for some function $\lambda \in \mathscr{A}_{\{x=0\}}^{\delta} \cap C^{1}(\bar{\Omega})$. This can be reduced to the case $\lambda=x$ by a change of variables

$$
(x, v, y) \longrightarrow(\lambda(x, v), v, y)
$$

which preserves the structure of the equations. We further note that the hypotheses of Theorem 3.1 are invariant under such coordinate transformations. From now on we assume that this change of variables has been made.

Theorem 3.1 Consider a set $\Omega$ as defined in (2.25), let $\alpha, \beta \in \mathbb{R}, k \in \mathbb{N}^{*}$, $\delta=1 / k$, and let $f=(\varphi, \psi) \in \mathscr{C}_{\{0 \leq x \leq y\}, \infty}^{\alpha}(\Omega)$ be a solution of (3.1). Suppose that

$$
L_{i j}=L_{i j}^{A} \partial_{A}+x L_{i j}^{y} \partial_{y}+x L_{i j}^{x} \partial_{x}
$$

with

$$
L_{\varphi \varphi}^{\mu} \in x^{\delta} \mathscr{A}_{\{0 \leq x \leq y\}}^{\delta}, \quad L_{\psi \varphi}^{\mu}, L_{\varphi \psi}^{\mu}, L_{\psi \psi}^{\mu} \in \mathscr{A}_{\{0 \leq x \leq y\}}^{\delta}
$$

(no symmetry hypotheses are made on the matrices $L_{i j}^{\mu}$ ), and

$$
\begin{aligned}
& B_{\varphi \varphi} \in C_{\infty}(\bar{\Omega})+x^{\delta} \mathscr{A}_{\{0 \leq x \leq y\}}^{\delta}, \quad B_{\varphi \psi}, B_{\psi \psi}, B_{\psi \varphi} \in \mathscr{A}_{\{0 \leq x \leq y\}}^{\delta}, \\
& a, b \in x^{\beta} \mathscr{A}_{\{0 \leq x \leq y\}}^{\delta}, \\
& \left.\varphi\right|_{\mathscr{S}}=\stackrel{\circ}{\varphi} \in x^{\beta} \mathscr{A}_{\{x=0\}}^{\delta},\left.\psi\right|_{\mathscr{S}}=\stackrel{\dot{\psi}}{\in} \in x^{\beta} \mathscr{A}_{\{x=0\}}^{\delta} .
\end{aligned}
$$

Then

$$
f \in x^{\beta} \mathscr{A}_{\{0 \leq x \leq y\}}^{\delta}+y^{\beta} \mathscr{A}_{\{0 \leq x \leq y\}}^{\delta}+\mathscr{A}_{\{x=0\}}^{\delta} .
$$

In particular for any $\tau>0$ we have $\left.f\right|_{\{y \geq \tau\}} \in x^{\beta} \mathscr{A}_{\{x=0\}}^{\delta}+\mathscr{A}_{\{x=0\}}^{\delta}$, so that the solution is polyhomogeneous with respect to $\{x=0\}$ on $\{y \geq \tau\}$.

REMARK 3.2 We have the more precise statement

$$
\begin{aligned}
& \psi \in x^{\beta+1} \mathscr{A}_{\{0 \leq x \leq y\}}^{\delta}+y^{\beta} \mathscr{A}_{\{y=0\}}^{\delta}+x y^{\beta} \mathscr{A}_{\{0 \leq x \leq y\}}^{\delta}+C_{\infty}(\bar{\Omega})+x \mathscr{A}_{\{x=0\}}^{\delta}, \\
& \varphi \in x^{\beta} \mathscr{A}_{\{x=0\}}^{\delta}+x^{\beta} y \mathscr{A}_{\{0 \leq x \leq y\}}^{\delta}+y^{\beta+1} \mathscr{A}_{\{0 \leq x \leq y\}}^{\delta}+x \mathscr{A}_{\{x=0\}}^{\delta}+y \mathscr{A}_{\{x=0\}}^{\delta} .
\end{aligned}
$$

Indeed, inserting (3.8) into the equations and integrating one obtains

$$
\begin{gathered}
\psi \in x^{\beta+1} \mathscr{A}_{\{0 \leq x \leq y\}}^{\delta}+y^{\beta} \mathscr{A}_{\{y=0\}}^{\delta}+x y^{\beta} \mathscr{A}_{\{0 \leq x \leq y\}}^{\delta}+x \mathscr{A}_{\{0 \leq x \leq y\}}^{\delta}+y \mathscr{A}_{\{y=0\}}^{\delta}, \\
\varphi \in x^{\beta} \mathscr{A}_{\{x=0\}}^{\delta}+x^{\beta} y \mathscr{A}_{\{0 \leq x \leq y\}}^{\delta}+x \mathscr{A}_{\{x=0\}}^{\delta}+y^{\beta+1} \mathscr{A}_{\{0 \leq x \leq y\}}^{\delta}+y \mathscr{A}_{\{0 \leq x \leq y\}}^{\delta} .
\end{gathered}
$$

Comparing this with (3.8) one obtains (3.9).

REMARK 3.3 It is of interest to enquire when the solutions are in $L^{\infty}$. A direct analysis of the equations and (3.9) lead to the following: First, $\psi$ will be in $L^{\infty}$ whenever $\beta>-1$ and $\dot{\psi} \in L^{\infty}$. Next, consider the case $\beta=0$. If

$$
L_{\varphi \psi}^{\mu}, B_{\varphi \psi}, a, \stackrel{\circ}{\varphi}, \stackrel{\circ}{\psi} \in L^{\infty},
$$

then it also holds that

$$
(\varphi, \psi) \in L^{\infty}
$$

Finally, for $\beta>0$, it suffices to assume that $L_{\varphi \psi}^{\mu}$ and $B_{\varphi \psi}$ are in $L^{\infty}$ to achieve (3.10). 
REMARK 3.4 In all physically relevant cases known to us we have $\beta \in-\delta \mathbb{N}$, in which case the two last terms in (3.8) can be absorbed in the first one, leading to the simpler formula

$$
f \in x^{\beta} \mathscr{A}_{\{0 \leq x \leq y\}}^{\delta} .
$$

Proof: By hypothesis we can decompose $B_{\varphi \varphi}$ as

$$
B_{\varphi \varphi}=\stackrel{\circ}{B}_{\varphi \varphi}+B_{\varphi \varphi}^{\delta}, \quad \stackrel{\circ}{B}_{\varphi \varphi} \in C_{\infty}(\bar{\Omega}), B_{\varphi \varphi}^{\delta} \in x^{\delta} \mathscr{A}_{\{0 \leq x \leq y\}}^{\delta} .
$$

We rewrite the equations at hand as

$$
\begin{aligned}
\partial_{y} \varphi+\stackrel{\circ}{B}_{\varphi \varphi} \varphi & =c_{\varphi}, \\
\partial_{x} \psi & =c_{\psi},
\end{aligned}
$$

where

$$
\begin{aligned}
& c_{\varphi}:=L_{\varphi \varphi} \varphi+L_{\varphi \psi} \psi+a-B_{\varphi \varphi}^{\delta} \varphi-B_{\varphi \psi} \psi, \\
& c_{\psi}:=L_{\psi \varphi} \varphi+L_{\psi \psi} \psi+b-B_{\psi \varphi} \varphi-B_{\psi \psi} \psi .
\end{aligned}
$$

Integration of the second equation yields

$$
\psi\left(x, v^{A}, y\right)=\underbrace{\psi\left(y, v^{A}, y\right)}_{\dot{\psi}\left(y, v^{A}\right)}+\int_{y}^{x} c_{\psi}\left(s, v^{A}, y\right) d s .
$$

By hypothesis we have $\stackrel{\circ}{\psi}\left(y, v^{A}\right) \in y^{\beta} \mathscr{A}_{\{y=0\}}^{\delta} \subset y^{\beta} \mathscr{A}_{\{0 \leq x \leq y\}}^{\delta}$. Similarly $\varphi$ can be calculated as

$$
\varphi\left(x, v^{A}, y\right)=R\left(x, v^{A} ; y, x\right) \underbrace{\varphi\left(x, v^{A}, x\right)}_{\dot{\varphi}\left(x, v^{A}\right)}+\int_{x}^{y} R\left(x, v^{A} ; s, x\right) c_{\varphi}\left(x, v^{A}, s\right) d s,
$$

where $R\left(x, v^{A} ; y, y_{1}\right)$ is the family of resolvents (smooth up to boundary in all variables) for the family of ODEs

$$
\partial_{y} \varphi\left(x, v^{A}, y\right)=-\stackrel{\circ}{B}_{\varphi \varphi}\left(x, v^{A}, y\right) \varphi\left(x, v^{A}, y\right),
$$

with parameters $\left(x, v^{A}\right)$ and with initial values at $y_{1}$. By hypothesis we have $\stackrel{\varphi}{\epsilon}$ $x^{\beta} \mathscr{A}_{\{x=0\}}^{\delta}$ which implies that the first term $R \dot{\varphi}$ is in $x^{\beta} \mathscr{A}_{\{x=0\}}^{\delta} \subset x^{\beta} \mathscr{A}_{\{0 \leq x \leq y\}}^{\delta}$.

In what follows we let $\epsilon>0$ be a positive constant, which can be made as small as desired, and which may change from line to line.

We wish, now, to show that

$$
f \in \mathscr{C}_{\{0 \leq x \leq y\}, \infty}^{-\epsilon}+x^{\beta} \mathscr{A}_{\{0 \leq x \leq y\}}^{\delta}+y^{\beta} \mathscr{A}_{\{0 \leq x \leq y\}}^{\delta} .
$$

If $\alpha \geq 0$ there is nothing to prove, so assume that $\alpha<0$. Decreasing $\alpha$ if necessary we can assume that $\alpha+k \delta \neq-1$ for all $k \in \mathbb{N}$. For all multi-indices $\gamma$ we have

$$
\partial_{v}^{\gamma} c_{f} \in \mathscr{C}_{\{0 \leq x \leq y\}, \infty}^{\alpha-\epsilon}+x^{\beta} \mathscr{A}_{\{0 \leq x \leq y\}}^{\delta}
$$


(here $\epsilon$ arises because of multiplicative powers of ln that might occur in some terms), in particular $\partial_{v}^{\gamma} \partial_{x} \psi \in \mathscr{C}_{\{0 \leq x \leq y\}, \infty}^{\alpha-\epsilon}+x^{\beta} \mathscr{A}_{\{0 \leq x \leq y\}}^{\delta}$, and by (3.12),

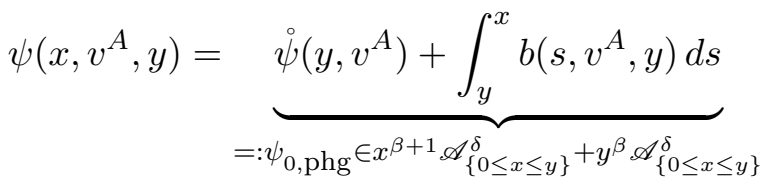

$$
\begin{aligned}
& +\int_{y}^{x} \underbrace{\left(L_{\psi \varphi} \varphi+L_{\psi \psi} \psi-B_{\psi \varphi} \varphi-B_{\psi \psi} \psi\right)\left(s, v^{A}, y\right)}_{O\left(s^{\alpha-\epsilon}\right)} d s \text {. }
\end{aligned}
$$

It follows that

$$
\psi-\psi_{0, \mathrm{phg}}=O\left(x^{\alpha+1-\epsilon}\right)+O\left(y^{\alpha+1-\epsilon}\right), \text { similarly for } \partial_{v}^{\gamma} \psi .
$$

The derivatives of $\psi$ with respect to $y$ take the form:

$\partial_{y}^{\ell} \psi\left(x, v^{A}, y\right)=\left(\partial_{x}^{\ell} \stackrel{\circ}{\psi}\right)\left(y, v^{A}\right)+\int_{y}^{x} \partial_{y}^{\ell} c_{\psi}\left(s, v^{A}, y\right) d s-\sum_{i=0}^{\ell-1} C_{i \ell}\left(\partial_{y}^{i} \partial_{x}^{\ell-1-i} c_{\psi}\right)\left(y, v^{A}, y\right)$

for some constants $C_{i \ell} \in \mathbb{N}$. Equation (3.18) with $\ell=1$ gives

$$
y \partial_{y}\left(\psi-\psi_{0, \mathrm{phg}}\right)=O\left(x^{\alpha+1-\epsilon}\right)+O\left(y^{\alpha+1-\epsilon}\right) \text {, similarly for } y \partial_{v}^{\gamma} \partial_{y} \psi .
$$

As $\delta \leq 1$, we have shown that

$$
\psi \in x^{\beta+1} \mathscr{A}_{\{0 \leq x \leq y\}}^{\delta}+y^{\beta} \mathscr{A}_{\{0 \leq x \leq y\}}^{\delta}+\mathscr{C}_{\{0 \leq x \leq y\}, 1}^{\min (\alpha+\delta-\epsilon)} .
$$

It now follows that

$$
c_{\varphi}=\underbrace{c_{\varphi, \text { phg }}}_{\in x^{\beta} \mathscr{A}_{\{0 \leq x \leq y\}}^{\delta}+y^{\beta} \mathscr{A}_{\{0 \leq x \leq y\}}^{\delta}}+\underbrace{c_{\varphi, \alpha+\delta-\epsilon}}_{O\left(x^{\alpha+\delta-\epsilon}\right)+O\left(y^{\alpha+\delta-\epsilon}\right)},
$$

and (3.13) gives

$$
\begin{aligned}
\varphi\left(x, v^{A}, y\right)= & \underbrace{R\left(x, v^{A} ; y, x\right) \dot{\varphi}\left(x, v^{A}\right)}_{\in x^{\beta} \mathscr{A}_{\{x=0\}}^{\delta}}+\underbrace{\int_{x}^{y} R\left(x, v^{A} ; s, x\right) c_{\varphi, \operatorname{phg}}\left(x, v^{A}, s\right) d s}_{\in x^{\beta} \mathscr{A}_{\{0 \leq x \leq y\}}^{\delta}+y^{\beta} \mathscr{A}_{\{0 \leq x \leq y\}}^{\delta}} \\
& +\int_{x}^{y} \underbrace{R\left(x, v^{A} ; s, x\right) c_{\varphi, \alpha+\delta-\epsilon}\left(x, v^{A}, s\right)}_{O\left(x^{\alpha+\delta-\epsilon}\right)+O\left(s^{\alpha+\delta-\epsilon}\right)} d s,
\end{aligned}
$$

leading to

$$
\begin{aligned}
\partial_{v}^{\gamma}\left(\varphi-\varphi_{0, \mathrm{phg}}\right) & =O\left(y x^{\alpha+\delta-\epsilon}\right)+O\left(x^{\alpha+\delta+1-\epsilon}\right)+O\left(y^{\alpha+\delta+1-\epsilon}\right) \\
& =O\left(x^{\min (\alpha+\delta-\epsilon, 0)}\right) \text { for } 0 \leq x \leq y
\end{aligned}
$$

with

$$
\varphi_{0, \operatorname{phg}}\left(x, v^{A}, y\right):=R\left(x, v^{A} ; y, x\right) \dot{\varphi}\left(x, v^{A}\right)+\int_{x}^{y} R\left(x, v^{A} ; s, x\right) c_{\varphi, \operatorname{phg}}\left(x, v^{A}, s\right) \text {. }
$$


Equations (3.19) and (3.20) in particular imply

$$
f \in x^{\beta} \mathscr{A}_{\{0 \leq x \leq y\}}^{\delta}+y^{\beta} \mathscr{A}_{\{0 \leq x \leq y\}}^{\delta}+\mathscr{C}_{\{0 \leq x \leq y\}, 0}^{\min (\alpha+\delta-\epsilon, 0)} .
$$

We note the following:

LEMMA 3.5 Under the hypotheses of Theorem 3.1, the fields

$$
\tilde{\varphi}:=\left(\begin{array}{c}
\varphi \\
\partial_{A} \varphi \\
x \partial_{x} \varphi \\
y \partial_{y} \varphi
\end{array}\right), \quad \tilde{\psi}:=\left(\begin{array}{c}
\psi \\
\partial_{A} \psi \\
x \partial_{x} \psi \\
y \partial_{y} \psi
\end{array}\right)
$$

satisfy a system of equations of the form (3.1), with coefficients $\tilde{L}_{i j}, \tilde{B}_{i j}$ and sources $\tilde{a}, \tilde{b}$ satisfying the hypotheses of Theorem 3.1, with $\tilde{\varphi}, \tilde{\psi} \in \mathscr{C}_{\{0 \leq x \leq y\}, \infty}^{\alpha}$, and with initial data $\left.\tilde{\varphi}\right|_{\mathscr{S}},\left.\tilde{\psi}\right|_{\mathscr{S}} \in x^{\beta} \mathscr{A}_{\{x=0\}}^{\delta}$.

PROOF: We rewrite the equations (3.1) as

$$
\begin{aligned}
\partial_{y} \varphi & =\stackrel{\circ}{\varphi}_{\varphi}, \\
\partial_{x} \psi & =c_{\psi} .
\end{aligned}
$$

Differentiating $(\tilde{\varphi}, \tilde{\psi})$ one gets

$$
\partial_{y} \tilde{\varphi}=\left(\begin{array}{c}
\stackrel{\circ}{c}_{\varphi} \\
\partial_{A} \stackrel{\circ}{\varphi}_{\varphi} \\
x \partial_{x} \stackrel{\circ}{\varphi}_{\varphi} \\
\stackrel{c}{c}_{\varphi}+y \partial_{y} \dot{c}_{\varphi}
\end{array}\right), \quad \partial_{x} \tilde{\psi}=\left(\begin{array}{c}
c_{\psi} \\
\partial_{A} c_{\psi} \\
c_{\psi}+x \partial_{x} c_{\psi} \\
y \partial_{y} c_{\psi}
\end{array}\right) .
$$

With a little work one shows that the right-hand sides of these equations are first order differential operators of appropriate form acting on $(\tilde{\varphi}, \tilde{\psi})$. For example, consider the term $y \partial_{y} \stackrel{\circ}{\varphi}_{\varphi}$ :

$$
\begin{aligned}
y \partial_{y} \stackrel{\circ}{\varphi}_{\varphi}= & y \partial_{y}\left(L_{\varphi \varphi} \varphi+L_{\varphi \psi} \psi+a-B_{\varphi \varphi} \varphi-B_{\varphi \psi} \psi\right) \\
= & \left(y \partial_{y} L_{\varphi \varphi}^{A}\right) \partial_{A} \varphi+\left(x \partial_{y} L_{\varphi \varphi}^{y}\right) y \partial_{y} \varphi+\left(y \partial_{y} L_{\varphi \varphi}^{x}\right) x \partial_{x} \varphi \\
& +L_{\varphi \varphi}^{A} \partial_{A}\left(y \partial_{y} \varphi\right)+L_{\varphi \varphi}^{y} x \partial_{y}\left(y \partial_{y} \varphi-\varphi\right)+L_{\varphi \varphi}^{x} x \partial_{x}\left(y \partial_{y} \varphi\right) \\
& +(\text { analogous } \partial \psi \text { terms }) \\
& +y \partial_{y} a-B_{\varphi \varphi}\left(y \partial_{y} \varphi\right)-B_{\varphi \psi}\left(y \partial_{y} \psi\right) \\
& -\left(y \partial_{y} B_{\varphi \varphi}\right) \varphi-\left(y \partial_{y} B_{\varphi \psi}\right) \psi
\end{aligned}
$$

The terms from the first two lines of the second equality above can be included in $\tilde{L}_{\tilde{\varphi} \tilde{\varphi}}$, the third line can be included in $\tilde{L}_{\tilde{\varphi} \tilde{\psi}}$, $\tilde{a}$ will include $y \partial_{y} a$, whatever remains going into the $\tilde{B}_{i j}$ terms. We note that $\mathscr{A}_{\{0 \leq x \leq y\}}^{\delta}$ and $x^{\delta} \mathscr{A}_{\{0 \leq x \leq y\}}^{\delta}$ are invariant under $y \partial_{y}$ and $x \partial_{x}$, which makes it simple to check that these choices lead to the functional spaces as required in (3.5)-(3.6).

The other terms in (3.23) are treated analogously. 
It remains to show that the initial data are in the right space. This is obvious for $\varphi, \psi$, as well as their derivatives with respect to $v^{A}$. For the remaining derivatives, we pass to the limit $x \rightarrow y$ in (3.18) with $\ell=1$ to obtain

$$
\left.y \partial_{y} \psi\right|_{\mathscr{S}}=y\left(\partial_{x} \dot{\psi}\right)-\left.y c_{\psi}\right|_{\mathscr{S}}
$$

Similarly from

$$
\varphi\left(x, v^{A}, y\right)=\underbrace{\varphi\left(x, v^{A}, x\right)}_{\dot{\varphi}\left(x, v^{A}\right)}+\int_{x}^{y} \stackrel{\circ}{c}_{\varphi}\left(x, v^{A}, s\right) d s .
$$

we find (again for $x=y$ )

$$
\left.y \partial_{x} \varphi\right|_{\mathscr{S}}=y\left(\partial_{x} \stackrel{\varphi}{)}-\left.y \stackrel{\circ}{\varphi}_{\varphi}\right|_{\mathscr{S}}\right.
$$

Equations (3.1) further give

$$
\begin{aligned}
& \left.y\left(\partial_{x} \psi\right)\right|_{\mathscr{S}}=\left.y c_{\psi}\right|_{\mathscr{S}} \\
& \left.y\left(\partial_{y} \varphi\right)\right|_{\mathscr{S}}=\left.y \dot{c}_{\varphi}\right|_{\mathscr{S}} .
\end{aligned}
$$

The terms $y\left(\partial_{x} \stackrel{\circ}{\psi}\right)\left(y, v^{A}\right)$ and $y\left(\partial_{x} \stackrel{\varphi}{\varphi}\right)\left(y, v^{A}\right)$ in $(3.24 \mathrm{a})-(3.24 \mathrm{~b})$ are in $y^{\beta} \mathscr{A}_{\{y=0\}}^{\delta}$. Now,

$$
\left.y \stackrel{\circ}{\varphi}_{\varphi}\right|_{\mathscr{S}}=\left.y\left(L_{\varphi \varphi} \varphi+L_{\varphi \psi} \psi+a-B_{\varphi \varphi} \varphi-B_{\varphi \psi} \psi\right)\right|_{\mathscr{S}} .
$$

The restrictions to $\mathscr{S}$ of the terms $a, B_{\varphi \varphi} \varphi, B_{\varphi \psi} \psi$ and the derivatives of $\varphi$ and $\psi$ with respect to $v^{A}$, give a contribution which is in $y^{\beta} \mathscr{A}_{\{y=0\}}^{\delta}$. The remaining terms are of the form $\left.y\left(\partial_{y} \psi\right)\right|_{\mathscr{S}},\left.y\left(\partial_{y} \varphi\right)\right|_{\mathscr{S}},\left.\left.y\left(\partial_{x} \psi\right)\right|_{\mathscr{S},} y\left(\partial_{x} \varphi\right)\right|_{\mathscr{S}}$ multiplied by coefficients from $\mathscr{A}_{\{y=0\}}^{\delta}$. The same analysis applies to $\left.y c_{\psi}\right|_{\mathscr{S}}$, so that we can write the system of equations (3.24) as

$$
(\mathrm{Id}-y K)\left(\begin{array}{l}
\left.y\left(\partial_{y} \psi\right)\right|_{\mathscr{S}} \\
\left.y\left(\partial_{y} \varphi\right)\right|_{\mathscr{S}} \\
\left.y\left(\partial_{x} \psi\right)\right|_{\mathscr{S}} \\
\left.y\left(\partial_{x} \varphi\right)\right|_{\mathscr{S}}
\end{array}\right) \in y^{\beta} \mathscr{A}_{\{y=0\}}^{\delta} .
$$

Here $K$ is a matrix with components in $\mathscr{A}_{\{y=0\}}^{\delta}$. There exists $\epsilon>0$ so that for $0 \leq y<\epsilon$ the matrix Id $-y K$ has an inverse in Id $+y \mathscr{A}_{\{y=0\}}^{\delta}$, and polyhomogeneity (with appropriate power structure) of the initial data for $(\tilde{\varphi}, \tilde{\psi})$ follows.

Applying (3.17) and $(3.20)$ to $(\tilde{\varphi}, \tilde{\psi})$ one finds that

$$
(\tilde{\varphi}, \tilde{\psi}) \in \mathscr{C}_{\{0 \leq x \leq y\}, 0}^{\min (\alpha+\delta-\epsilon, 0)}+x^{\beta} \mathscr{A}_{\{0 \leq x \leq y\}}^{\delta}+y^{\beta} \mathscr{A}_{\{0 \leq x \leq y\}}^{\delta},
$$

and Proposition A.5 implies

$$
(\varphi, \psi) \in \mathscr{C}_{\{0 \leq x \leq y\}, 1}^{\min (\alpha+\delta-\epsilon,-\epsilon)}+x^{\beta} \mathscr{A}_{\{0 \leq x \leq y\}}^{\delta}+y^{\beta} \mathscr{A}_{\{0 \leq x \leq y\}}^{\delta} .
$$


Continuing in this way we are led to

$$
(\varphi, \psi) \in \mathscr{C}_{\{0 \leq x \leq y\}, \infty}^{\min (\alpha+\delta-\epsilon,-\epsilon)}+x^{\beta} \mathscr{A}_{\{0 \leq x \leq y\}}^{\delta}+y^{\beta} \mathscr{A}_{\{0 \leq x \leq y\}}^{\delta} .
$$

Repeating the whole argument $\ell$ times, where $\ell$ is the smallest number such that $\alpha+\ell \delta>0$, one arrives at

$$
f \in \mathscr{C}_{\{0 \leq x \leq y\}, \infty}^{-\epsilon}+x^{\beta} \mathscr{A}_{\{0 \leq x \leq y\}}^{\delta}+y^{\beta} \mathscr{A}_{\{0 \leq x \leq y\}}^{\delta} .
$$

Suppose, for the sake of induction, that

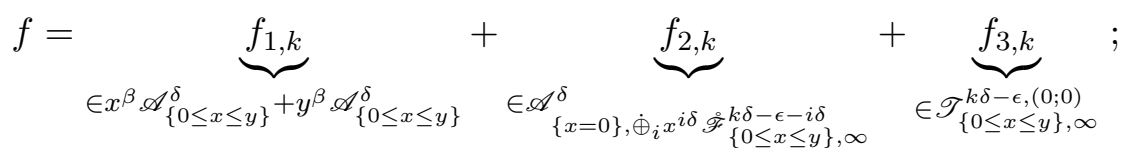

Equation (3.26) and the embedding

$$
\mathscr{C}_{\{0 \leq x \leq y\}, \infty}^{-\epsilon} \subset \mathscr{T}_{\{0 \leq x \leq y\}, \infty}^{-\epsilon,(0 ; 0)}
$$

show that this equation holds with $k=0$ and $f_{2,0}=0$. We note that all of the above spaces are invariant with respect to $x \partial_{x}, x \partial_{y}, \partial_{v}$ and with respect to multiplication by a function from $\mathscr{A}_{\{0 \leq x \leq y\}}^{\delta}$.

Integrating the equation for $\psi$ and using Propositions A.9, A.12 and A.14 one finds

$$
\begin{aligned}
& \psi\left(x, v^{A}, y\right)=\underbrace{\psi_{0, \operatorname{phg}}\left(x, v^{A}, y\right)+I_{1}\left(L_{\psi \varphi} \varphi_{1, k}+L_{\psi \psi} \psi_{1, k}-B_{\psi \varphi} \varphi_{1, k}-B_{\psi \psi} \psi_{1, k}\right)} \\
& =: \psi_{1, k+1} \in x^{\beta+1} \mathscr{A}_{\{0 \leq x \leq y\}}^{\delta}+y^{\beta} \mathscr{A}_{\{0 \leq x \leq y\}}^{\delta}
\end{aligned}
$$

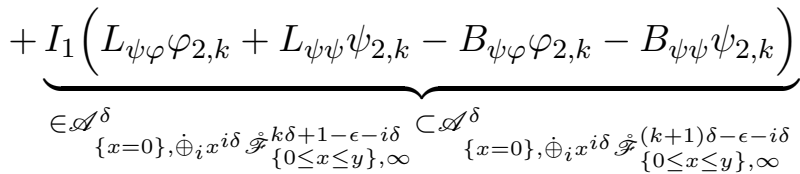

$$
\begin{aligned}
& +\underbrace{I_{1}\left(L_{\psi \varphi} \varphi_{3, k}+L_{\psi \psi} \psi_{3, k}-B_{\psi \varphi} \varphi_{3, k}-B_{\psi \psi} \psi_{3, k}\right)} \\
& \in \mathscr{F}_{\{0 \leq x \leq y\}, \infty}^{k \delta+1-\epsilon}+\mathscr{T}_{\{0 \leq x \leq y\}, \infty}^{k \delta+1-\epsilon,(0 ; 0)} \subset \mathscr{F}_{\{0 \leq x \leq y\}, \infty}^{(k+1) \delta-\epsilon}+\mathscr{T}_{\{0 \leq x \leq y\}, \infty}^{(k+1) \delta-\epsilon,(0 ; 0)}
\end{aligned}
$$

showing that the result is true for $\psi$ with $k$ replaced by $k+1$. Inserting this information into (3.13) one similarly finds, using Propositions A.9 and A.15, that (3.27) with $k$ replaced by $k+1$ holds for $\varphi$ (here $R$ stands for the smooth up to the boundary - resolvent $\left.R\left(x, v^{A} ; y, x\right)\right)$ :

$$
\begin{aligned}
& \varphi\left(x, v^{A}, y\right)=\underbrace{\varphi_{0, \operatorname{phg}}\left(x, v^{A}, y\right)+I_{2}\left[R \cdot\left(L_{\varphi \varphi} \varphi_{1, k}+L_{\varphi \psi} \psi_{1, k}-B_{\varphi \varphi}^{\delta} \varphi_{1, k}-B_{\varphi \psi} \psi_{1, k}\right)\right]}_{=: \varphi_{1, k+1} \in x^{\beta} \mathscr{A}_{\{0 \leq x \leq y\}}^{\delta}+y^{\beta} \mathscr{A}_{\{0 \leq x \leq y\}}^{\delta}} \\
& +\underbrace{I_{2}\left[R \cdot\left(L_{\varphi \varphi} \varphi_{2, k}+L_{\varphi \psi} \psi_{2, k}-B_{\varphi \varphi}^{\delta} \varphi_{2, k}-B_{\varphi \psi} \psi_{2, k}\right)\right]}_{\mathcal{A}_{\{x=0\}, \dot{\oplus}_{i} x^{i \delta} \mathscr{F}^{(k+1) \delta-i \delta}} \underbrace{}_{\{0 \leq x \leq y\}, \infty}} \\
& +I_{2}\left[R \cdot(\underbrace{L_{\varphi \varphi} \varphi_{3, k}-B_{\varphi \varphi}^{\delta} \varphi_{3, k}+L_{\varphi \psi} \psi_{3, k}-B_{\varphi \psi} \psi_{3, k}}_{\in \mathscr{T}_{\{0 \leq x \leq y\}, \infty}^{(k+1) \delta-\epsilon,(0 ; 0)}})\right] . \\
& \in \mathscr{T}_{\{0 \leq x \leq y\}, \infty}^{(k+1) \delta-\epsilon,(1 ; 1)} \subset \mathscr{T}_{\{0 \leq x \leq y\}, \infty}^{(k+1) \delta-\epsilon,(0 ; 0)}
\end{aligned}
$$


We have thus shown that (3.27) holds for all $k$. For any $m \in \mathbb{N}$ we can choose $k$ large enough so that the last term there is in $C_{m}(\bar{\Omega})$, and that all the coefficients of an expansion of $f_{2, k}$ in terms of powers of $x$ and $\log x$ also are in $C_{m}(\bar{\Omega})$. The result follows now by arguments essentially identical to those of Proposition A.3.

REMARK 3.6 An identical proof shows that "any" polyhomogeneous initial data, for equations with "any" polyhomogeneous and bounded coefficients, and "any" polyhomogeneous source terms lead to polyhomogeneous solutions, without imposing corner conditions. More precisely, let us assume, instead of (3.5), that there exists $\lambda>0$ such that

$$
L_{\varphi \varphi}^{\mu} \in x^{\lambda} \mathscr{A}_{\{0 \leq x \leq y\}}^{+}, \quad L_{\psi \varphi}^{\mu}, L_{\varphi \psi}^{\mu}, L_{\psi \psi}^{\mu} \in \mathscr{A}_{\{0 \leq x \leq y\}}^{+} .
$$

By $\mathscr{A}_{\{0 \leq x \leq y\}}^{+}, \mathscr{A}_{\{x=0\}}^{+}$, etc. we mean here spaces of polyhomogeneous functions for which $n_{i} \geq 0$ and $n_{i}+\hat{n}_{i} \geq 0$, where $n_{i}$ and $\hat{n}_{i}$ denote the powers of, respectively, $x$ and $y$ in the polyhomogeneous expansion. Let us further assume that, for some $\lambda^{\prime}>0$,

$$
\begin{aligned}
& B_{\varphi \varphi} \in C_{\infty}(\bar{\Omega})+x^{\lambda^{\prime}} \mathscr{A}_{\{0 \leq x \leq y\}}^{+}, \quad B_{\varphi \psi}, B_{\psi \psi}, B_{\psi \varphi} \in \mathscr{A}_{\{0 \leq x \leq y\}}^{+}, \\
& a \in x^{\beta_{1}} \mathscr{A}_{\{0 \leq x \leq y\}}^{+}, \quad b \in x^{\beta_{2}} \mathscr{A}_{\{0 \leq x \leq y\}}^{+}, \\
& \left.\varphi\right|_{\mathscr{S}}=\stackrel{\varphi}{\varphi} \in x^{\beta_{3}} \mathscr{A}_{\{x=0\}}^{+},\left.\psi\right|_{\mathscr{S}}=\dot{\psi} \in x^{\beta_{4}} \mathscr{A}_{\{x=0\}}^{+} .
\end{aligned}
$$

We have then the following version of the result:

$$
f \in y^{\beta} \mathscr{A}_{\{0 \leq x \leq y\}}^{+}+x^{\beta} \mathscr{A}_{\{0 \leq x \leq y\}}^{+}+\mathscr{A}_{\{x=0\}}^{+},
$$

where $\beta=\min \left(\beta_{1}+1, \beta_{2}, \beta_{3}, \beta_{4}\right)$. The conclusion of Remark 3.3 remains valid if $\beta$ there is replaced with $\min \left(\beta_{1}+1, \beta_{2}, \beta_{3}, \beta_{4}\right)$.

\subsection{Nonlinear equations}

In this section we prove the non-linear equivalent of Theorem 3.1. The method of proof is essentially identical, when the nonlinearities are treated appropriately. We consider semi-linear systems of the form (3.1) with a supplementary non-linearity at the right-hand-side:

$$
\begin{aligned}
\partial_{y} \varphi+B_{\varphi \varphi} \varphi+B_{\varphi \psi} \psi & =L_{\varphi \varphi} \varphi+L_{\varphi \psi} \psi+a+G_{\varphi} \\
\partial_{x} \psi+B_{\psi \varphi} \varphi+B_{\psi \psi} \psi & =L_{\psi \varphi} \varphi+L_{\psi \psi} \psi+b+G_{\psi}
\end{aligned}
$$

It should be clear from the discussion at the beginning of Section 3.1 that the right hypothesis for a consistent set-up as in Theorem 3.1 is to assume $\beta=-1+\delta$, and to have a non-linearity compatible with this hypothesis. This leads us to assume, following [11], that the nonlinearity

$$
G=\left(G_{\varphi}, G_{\psi_{1}}, G_{\psi_{2}}\right)
$$


takes the form

$$
G=x^{-p \delta} H\left(x^{\mu}, x^{q \delta} \psi_{1}, x^{q \delta+1} \psi_{2}, x^{q \delta+1} \varphi\right) .
$$

Here we have decomposed $\psi$ as

$$
\psi=\left(\begin{array}{c}
\psi_{1} \\
\psi_{2}
\end{array}\right)
$$

this is motivated by different a priori estimates we have at our disposal for the appropriately defined components $\psi_{1}$ and $\psi_{2}$ of $\psi$ in the applications we have in mind. Polyhomogeneity of solutions of nonlinear wave equations, or of the wave map equation, follows as a special case, see the proofs of Theorem 1.1 and of Theorem 1.2.

We will need to impose various restrictions on the function $H$, in order to do that some terminology will be needed. We shall say that a function $H\left(x^{\mu}, w\right)$ is $\mathscr{A}_{\{0 \leq x \leq y\}}^{\delta}$-polyhomogeneous in $x^{\mu}$ with a uniform zero of order $l$ in $w$ if the following hold: First, $H$ is smooth in $w \in \mathbb{R}^{N}$ at fixed $x^{\mu}$. Next, it is required that for all $B \in \mathbb{R}$ and $k \in \mathbb{N}$ there exists a constant $\hat{C}(B)$ such that for all $|w| \leq B$ and $0 \leq i \leq \min (k, l)$,

$$
\left\|\frac{\partial^{i} H(\cdot, w)}{\partial w^{i}}\right\|_{\mathscr{C}_{k-i}^{0}(\Omega)} \leq \hat{C}(B)|w|^{l-i} .
$$

Further,

$$
\forall i \in \mathbb{N} \quad \partial_{w}^{i} H(\cdot, w) \in \mathscr{A}_{\{0 \leq x \leq y\}}^{\delta}
$$

at fixed constant $w$. Finally we demand the uniform estimate for constant $w$ 's

$\forall \epsilon>0, M \geq 0, i, k \in \mathbb{N} \exists C(\epsilon, M, i, k) \forall|w| \leq M \quad\left\|\partial_{w}^{i} H(\cdot, w)\right\|_{\mathscr{C}_{k}^{-\epsilon}(\Omega)} \leq C(\epsilon, M, i, k)$.

The qualification "in $w$ " in "uniform zero of order $l$ in $w$ " will often be omitted. The small parameter $\epsilon$ has been introduced above to take into account the possible logarithmic blow-up of functions in $\mathscr{A}_{\{0 \leq x \leq y\}}^{\delta}$ at $x=0$; for the applications to the nonlinear scalar wave equation or to the wave map equation on Minkowski space-time, the alternative simpler requirement would actually suffice:

$$
\forall M \geq 0, i, k \in \mathbb{N} \exists C(M, i, k) \forall|w| \leq M \quad\left\|\partial_{w}^{i} H(\cdot, w)\right\|_{\mathscr{C}_{k}^{0}(\Omega)} \leq C(M, i, k),
$$

again for constant $w$ 's. Clearly functions which are smooth in $\left(w, x^{\mu}\right)$, and have a zero of appropriate order in $w$ at $w=0$, satisfy the above conditions.

Theorem 3.7 Let $p \in \mathbb{Z}, q, 1 / \delta \in \mathbb{N}^{*}, k \in \mathbb{N} \cup\{\infty\}$, let $f=\left(\psi=\left(\psi_{1}, \psi_{2}\right), \varphi\right)$ be a solution of (3.35) with $G$ of the form (3.36), where $H$ is $\mathscr{A}_{\{0 \leq x \leq y\}}^{\delta}$-polyhomogeneous in $x^{\mu}$ with a uniform zero of order

$$
m>\frac{p-\frac{1}{\delta}}{q} .
$$


Assume that for every $\epsilon>0$ we have

$$
\begin{gathered}
\psi_{1} \in\left(\mathscr{C}_{\{0 \leq x \leq y\}, \infty}^{-1-\epsilon} \cap \mathscr{C}_{\{0 \leq x \leq y\}, 0}^{-\epsilon}\right)(\Omega), \\
\varphi, \psi_{2} \in \mathscr{C}_{\{0 \leq x \leq y\}, \infty}^{-1-\epsilon}(\Omega) .
\end{gathered}
$$

Suppose that Equations (3.4)-(3.7) hold with $\beta=-1+\delta$, and that $\left.\psi\right|_{x=y} \in$ $\mathscr{A}_{\{x=0\}}^{\delta}$. Then

$$
(\psi, \varphi) \in \mathscr{A}_{\{0 \leq x \leq y\}}^{\delta} \times x^{\delta-1} \mathscr{A}_{\{0 \leq x \leq y\}}^{\delta}
$$

more precisely

$$
\begin{aligned}
& \psi \in x^{\delta} \mathscr{A}_{\{0 \leq x \leq y\}}^{\delta}+\mathscr{A}_{\{y=0\}}^{\delta}, \\
& \varphi \in x^{\delta-1} \mathscr{A}_{\{x=0\}}^{\delta}+x^{\delta-1} y \mathscr{A}_{\{0 \leq x \leq y\}}^{\delta} .
\end{aligned}
$$

In particular for any $\tau>0$ we have

$$
\left.(\psi, \varphi)\right|_{\{y \geq \tau\}} \in \mathscr{A}_{\{x=0\}}^{\delta} \times x^{\delta-1} \mathscr{A}_{\{x=0\}}^{\delta},
$$

which shows that the solution is polyhomogeneous with respect to $\{x=0\}$ on $\{y \geq \tau\}$.

REMaRK 3.8 If $\left.\psi\right|_{x=y} \in \mathscr{A}_{\{x=0\}}^{\delta} \cap L^{\infty}$, then $\psi \in x^{\delta} \mathscr{A}_{\{0 \leq x \leq y\}}^{\delta}+\mathscr{A}_{\{y=0\}}^{\delta} \cap L^{\infty}$.

REMARK 3.9 Let us, in addition to $\psi \in L^{\infty}(\bar{\Omega})$ (see Remark 3.8), assume that

$$
L_{\varphi \psi}^{\mu}, B_{\varphi \psi}, a,\left.\varphi\right|_{x=y} \in L^{\infty}(\bar{\Omega}) .
$$

Suppose further that $G_{\varphi}$ satisfies

$$
G_{\varphi}\left(x^{\mu}, x^{q \delta} \psi_{1}, x^{q \delta+1} \psi_{2}, x^{q \delta+1} \varphi\right) \in L^{\infty}(\bar{\Omega})
$$

for every (fixed) $\psi, \varphi$ as in (3.43) (which is true e.g. if $(m q-p) \delta>0)$. Then it holds that

$$
\varphi \in \mathscr{A}_{\{0 \leq x \leq y\}}^{\delta} \cap L^{\infty}(\bar{\Omega}) .
$$

REMARK 3.10 There is little doubt that the proof below can be generalised to allow $\psi_{2} \in x^{-1+\delta} \mathscr{A}_{\{x=0\}}^{\delta}$, as well as $B_{\psi \psi} \in x^{-1+\delta} \mathscr{A}_{\{x=0\}}^{\delta}$. We have not attempted to check all the details of this, because of technical complications in the proof.

REMARK 3.11 The theorem remains true if we replace $G$ by a finite sum of nonlinearities satisfying the above hypotheses, with different $p$ and $q$ satisfying (3.42) for each term in the sum.

Proof: We follow the proof of Theorem 3.1, checking that the supplementary non-linear terms do not affect the argument. We decompose $c_{\psi}$ as $\left(c_{\psi_{1}}, c_{\psi_{2}}\right)$.

We have

$$
\widetilde{f}:=\left(\psi_{1}, x \psi_{2}, x \varphi\right) \in \mathscr{C}_{\{0 \leq x \leq y\}, 0}^{-\epsilon},
$$


with $\epsilon$ as small as desired (we hope that a clash of notation $\tilde{f}$ with Section 2 will not confuse the reader). This shows that the non-linearity $G_{\psi}$ gives a contribution to $c_{\psi}$ which is $O\left(x^{(m q-p) \delta-\epsilon}\right)$ (the easiest way to see that is to view the non-linearity as a function of $x^{q \delta-\epsilon} \widetilde{f}_{\epsilon}$, where $\left.\tilde{f}_{\epsilon}=x^{\epsilon} \tilde{f} \in L^{\infty}\right)$.

First, (3.17) becomes

$$
\psi-\psi_{0, \mathrm{phg}}=O\left(x^{-\epsilon}\right) .
$$

Next, to estimate the derivatives of $\psi$, note that the integrand for $\partial_{y} \psi$ in (3.18) with $\ell=1$ contains a supplementary term

$$
\partial_{y}\left(G_{\psi}\left(x^{\mu}, x^{q \delta} \tilde{f}\right)\right)=\underbrace{\left(\partial_{y} G_{\psi}\right)\left(x^{\mu}, x^{q \delta} \tilde{f}\right)}_{O\left(x^{(m q-p) \delta-\epsilon}\right) y^{-1}}+\left(\partial_{w} G_{\psi}\right)\left(x^{\mu}, x^{q \delta} \tilde{f}\right) x^{q \delta} \partial_{y} \tilde{f} .
$$

Write $w=\left(w_{1}, w_{2}, w_{\varphi}\right)=\left(x^{q \delta} \psi_{1}, x^{q \delta+1} \psi_{2}, x^{q \delta+1} \varphi\right), w_{\psi}=\left(w_{1}, w_{2}\right)$. We have the equation

$$
\begin{aligned}
\left(\partial_{w} G_{\psi}\right)\left(x^{\mu}, x^{q \delta} \widetilde{f}\right) x^{q \delta} \partial_{y} \tilde{f}= & x^{-p \delta}\left(\partial_{w_{2}} H_{\psi}\right)\left(x^{\mu}, w\right) x^{q \delta+1} \partial_{y} \psi_{2} \\
& +x^{-p \delta}\left(\partial_{w_{\varphi}} H_{\psi}\right)\left(x^{\mu}, w\right) x^{q \delta+1} \partial_{y} \varphi \\
& +x^{-p \delta}\left(\partial_{w_{1}} H_{\psi}\right)\left(x^{\mu}, w\right) x^{q \delta} \partial_{y} \psi_{1}
\end{aligned}
$$

We know by hypothesis that

$$
\left(\psi_{1}, x \psi_{2}, x \varphi, x y \partial_{y} \psi_{2}, x y \partial_{y} \varphi\right) \in \mathscr{C}_{\{0 \leq x \leq y\}, 0}^{-\epsilon},
$$

which allows us to estimate all terms above by $C x^{(m q-p) \delta-\epsilon} y^{-1}$, except the last one. However, we can write a system of equations for $y \partial_{y} \psi$ of the form:

$$
\partial_{x}\left(y \partial_{y} \psi\right)-\underbrace{x^{-p \delta}\left(\partial_{w_{1}} H_{\psi}\right)\left(x^{\mu}, w\right) x^{q \delta}}_{=O\left(x^{(m q-p) \delta-\epsilon}\right)} y \partial_{y} \psi_{1}=O\left(x^{-1-\epsilon}\right) .
$$

The following is essentially Proposition B.3 of [11] with $k=0$ there, we revisit the proof because of the uniformity in $y$ required here:

Lemma 3.12 For $0 \leq x \leq y \leq \dot{y}$ consider the system

$$
\partial_{x} \psi+b \psi=c,
$$

and suppose that there exists $\epsilon<1$ such that the linear map $b$ has coefficients in $\mathscr{C}_{\{x=0\}, 0}^{-\epsilon}$. For $\alpha \in \mathbb{R} \backslash\{-1\}$ there exists a constant $C=C\left(\alpha, \epsilon,\|b\|_{\mathscr{C}_{\{x=0\}, 0}^{-\epsilon}}\right.$, ํ) such that

1. For $\alpha>-1$ we have

$$
\|\psi\|_{L^{\infty}} \leq C\left(\left\|\left.\psi\right|_{x=y}\right\|_{L^{\infty}}+\|c\|_{\mathscr{C}_{\{x=0\}, 0}^{\alpha}}\right)
$$


2. while for $\alpha<-1$ it holds that

$$
\|\psi\|_{\mathscr{C}_{\{x=0\}, 0}^{\alpha+1}} \leq C\left(\left\|\left.\psi\right|_{x=y}\right\|_{\mathscr{C}_{\{x=0\}, 0}^{\alpha+1}}+\|c\|_{\mathscr{C}_{\{x=0\}, 0}^{\alpha}}\right)
$$

Proof: 1. Obvious modifications in the calculations in the proof of [11, Proposition B.3] lead to the following replacement of [11, Equation (B.19)]

$$
-\partial_{x} e_{a} \leq x^{\alpha}\|c\|_{\mathscr{C}_{\{x=0\}, k}^{\alpha}}+x^{\epsilon}\|b \psi\|_{\mathscr{C}_{\{x=0\}, k}^{-\epsilon}} .
$$

By integration, after passing to the limit $a \rightarrow 0$, one obtains, for $0 \leq x \leq y \leq$ $y_{1} \leq \dot{y}$

$$
\|\psi\|_{L^{\infty}\left(\left[0, y_{1}\right]\right)} \leq\left\|\left.\psi\right|_{x=y}\right\|_{L^{\infty}}+\frac{\stackrel{\circ}{y}^{\alpha+1}}{|\alpha+1|}\|c\|_{\mathscr{C}_{\{x=0\}, k}^{\alpha}}+\frac{y_{1}^{1-\epsilon}}{1-\epsilon}\|b\|_{\mathscr{C}_{\{x=0\}, k}^{-\epsilon}}\|\psi\|_{L^{\infty}\left(\left[0, y_{1}\right]\right)} .
$$

Choosing $y_{1}$ small enough the last term can be carried over to the left-handside, which yields the inequality on $\left[0, y_{1}\right]$. The final inequality $(3.46)$ is then standard.

2. Decreasing $\epsilon$ slightly if necessary, we can without loss of generality assume that $\alpha+1-\epsilon \neq-1$. We recall the first two lines of [11, Equation (B.20)], adapted to our notation, with $x_{3}$ there replaced by $y$ :

$$
\begin{aligned}
\left|\psi\left(x, v^{A}, y\right)\right| \leq & \left|\psi\left(y, v^{A}, y\right)\right|+\left(-\frac{x^{\alpha+1}}{(1+\alpha)}+\frac{y^{\alpha+1}}{(1+\alpha)}\right)\|c\|_{\mathscr{C}_{\{x=0\}, 0}^{\alpha}} \\
& +\left(\frac{y^{\alpha+2-\epsilon}}{(2+\alpha-\epsilon)}-\frac{x^{\alpha+2-\epsilon}}{(2+\alpha-\epsilon)}\right)\|b\|_{\mathscr{C}_{\{x=0\}, 0}^{-\epsilon}}\|\psi\|_{\mathscr{C}_{\{x=0\}, 0}^{\alpha+1}}
\end{aligned}
$$

For $\alpha<-1$ this implies

$$
\begin{aligned}
& \left|\psi\left(x, v^{A}, y\right)\right| \leq\left\|\psi\left(y, v^{A}, y\right)\right\|_{\mathscr{C}_{\{x=0\}, 0}^{\alpha+1}} \underbrace{y^{\alpha+1}}_{\leq x^{\alpha+1}}+\frac{x^{\alpha+1}}{|1+\alpha|}\|c\|_{\mathscr{C}_{\{x=0\}, 0}^{\alpha}} \\
& +\frac{1}{|2+\alpha-\epsilon|}(\underbrace{y^{\alpha+2-\epsilon}}_{=y^{1-\epsilon} y^{\alpha+1} \leq y_{1}^{1-\epsilon} x^{\alpha+1}}+\underbrace{x^{\alpha+2-\epsilon}}_{=x^{1-\epsilon} x^{\alpha+1} \leq y_{1}^{1-\epsilon} x^{\alpha+1}})\|b\|_{\mathscr{C}_{\{x=0\}, 0}^{-\epsilon}}\|\psi\|_{\mathscr{C}_{\{x=0\}, 0}^{\alpha+1}} \\
& \leq\left(\left\|\psi\left(y, v^{A}, y\right)\right\|_{\mathscr{C}_{\{x=0\}, 0}^{\alpha+1}}+\frac{1}{|1+\alpha|}\|c\|_{\mathscr{C}_{\{x=0\}, 0}^{\alpha}}\right. \\
& \left.+\frac{2 y_{1}^{1-\epsilon}}{|2+\alpha-\epsilon|}\|b\|_{\mathscr{C}_{\{x=0\}, 0}^{-\epsilon}}\|\psi\|_{\mathscr{C}_{\{x=0\}, 0}^{\alpha+1}}\right) x^{\alpha+1} .
\end{aligned}
$$

Choosing $y_{1}$ sufficiently small, one concludes as before.

From (3.45a) and Lemma 3.12 with $\alpha=-1-\epsilon$ we obtain

$$
y \partial_{y} \psi=O\left(x^{-\epsilon}\right) .
$$

A similar treatment of $\partial_{A} G_{\psi}$ allows one to conclude that

$$
\psi \in x^{\delta} \mathscr{A}_{\{0 \leq x \leq y\}}^{\delta}+\mathscr{A}_{\{y=0\}}^{\delta}+\mathscr{C}_{\{0 \leq x \leq y\}, 1}^{-\epsilon} .
$$


(The first two spaces are actually included in the last one, but we exhibit them to keep track of the form of the polyhomogeneous contributions in $\psi$, which will become important later.) It now follows that

$$
c_{\varphi}+G_{\varphi}=\underbrace{c_{\varphi, \text { phg }}}_{\in x^{\beta} \mathscr{A}_{\{0 \leq x \leq y\}}^{\delta}+y^{\beta} \mathscr{A}_{\{0 \leq x \leq y\}}^{\delta}}+c_{\varphi}^{\prime},
$$

with

$$
c_{\varphi}^{\prime}=O\left(x^{-1+\delta-\epsilon}\right)
$$

Equation (3.13) gives

$$
\begin{aligned}
\varphi\left(x, v^{A}, y\right)= & \underbrace{R\left(x, v^{A} ; y, x\right) \dot{\varphi}\left(x, v^{A}\right)}_{\in x^{\delta-1} \mathscr{A}_{\{x=0\}}^{\delta}}+\underbrace{\int_{x}^{y} R\left(x, v^{A} ; s, x\right) c_{\varphi, \operatorname{phg}}\left(x, v^{A}, s\right) d s}_{\in x^{\delta} \mathscr{A}_{\{x=0\}}^{\delta}+x^{\delta-1} y \mathscr{A}_{\{0 \leq x \leq y\}}^{\delta}} \\
& +\int_{x}^{y} R\left(x, v^{A} ; s, x\right) c_{\varphi}^{\prime}\left(x, v^{A}, s\right) d s,
\end{aligned}
$$

leading to

$$
\varphi-\varphi_{0, \mathrm{phg}}=O\left(x^{-1+\delta-\epsilon}\right) .
$$

Here, as before, $\varphi_{0, \text { phg }}$ is given by $(3.21)$.

Next, consider Lemma 3.5. We rewrite (3.1) as

$$
\begin{aligned}
& \partial_{y} \varphi=\stackrel{\circ}{c}_{\varphi}+G_{\varphi}, \\
& \partial_{x} \psi=c_{\psi}+G_{\psi} .
\end{aligned}
$$

The field $\tilde{\psi}$ is decomposed as $\left(\tilde{\psi}_{1}, \tilde{\psi}_{2}\right)$, with $\tilde{\psi}_{1}=\left(\psi_{1}, \partial_{A} \psi_{1}, x \partial_{x} \psi_{1}, y \partial_{y} \psi_{1}\right)$. Differentiating $(\tilde{\varphi}, \tilde{\psi})$, instead of $(3.23)$ one gets

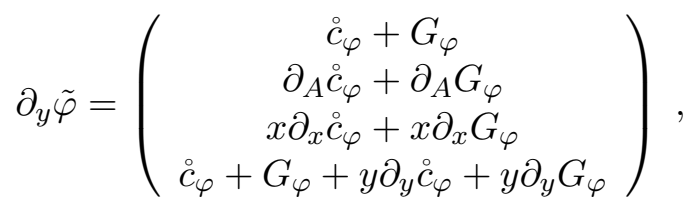

similarly for $\partial_{x} \tilde{\psi}$, e.g.

$$
\partial_{x} \tilde{\psi}_{1}=\left(\begin{array}{c}
c_{\psi_{1}}+G_{\psi_{1}} \\
\partial_{A} c_{\psi_{1}}+\partial_{A} G_{\psi_{1}} \\
c_{\psi_{1}}+G_{\psi_{1}}+x \partial_{x} c_{\psi_{1}}+x \partial_{x} G_{\psi_{1}} \\
y \partial_{y} c_{\psi_{1}}+y \partial_{y} G_{\psi_{1}}
\end{array}\right) .
$$

One can check that the non-linear terms above have the structure claimed. For example, we have

$$
\begin{aligned}
x \partial_{x} G_{\psi_{1}}= & x \partial_{x}\left(x^{-p \delta} H_{\psi_{1}}\left(x^{\mu}, w\right)\right) \\
= & -p x^{-p \delta} H_{\psi_{1}}\left(x^{\mu}, w\right)+x^{1-p \delta}\left(\partial_{x} H_{\psi_{1}}\right)\left(x^{\mu}, w\right) \\
& +x^{-p \delta}\left(\partial_{w_{1}} H_{\psi_{1}}\right)\left(x^{\mu}, w\right)\left(q \delta x^{q \delta} \psi_{1}+x^{q \delta} x \partial_{x} \psi_{1}\right) \\
& +x^{-p \delta}\left(\partial_{w_{2}} H_{\psi_{1}}\right)\left(x^{\mu}, w\right)\left((q \delta+1) x^{q \delta+1} \psi_{2}+x^{q \delta+1} x \partial_{x} \psi_{2}\right) \\
& +x^{-p \delta}\left(\partial_{w_{\varphi}} H_{\psi_{1}}\right)\left(x^{\mu}, w\right)\left((q \delta+1) x^{q \delta+1} \varphi+x^{q \delta+1} x \partial_{x} \varphi\right) .
\end{aligned}
$$


It should be clear that each term in the sum above has a zero of order $m$ in the new variables.

This analysis allows us to repeat the induction argument which led to (3.25), obtaining instead

$$
\begin{aligned}
& \psi \in x^{\delta} \mathscr{A}_{\{0 \leq x \leq y\}}^{\delta}+\mathscr{A}_{\{y=0\}}^{\delta}+\mathscr{C}_{\{0 \leq x \leq y\}, \infty}^{-\epsilon}, \\
& \varphi \in x^{\delta-1} \mathscr{A}_{\{x=0\}}^{\delta}+x^{\delta-1} y \mathscr{A}_{\{0 \leq x \leq y\}}^{\delta}+\mathscr{C}_{\{0 \leq x \leq y\}, \infty}^{-1+\delta-\epsilon} .
\end{aligned}
$$

The embeddings

$$
\mathscr{C}_{\{0 \leq x \leq y\}, \infty}^{-\epsilon} \subset \mathscr{T}_{\{0 \leq x \leq y\}, \infty}^{-\epsilon,(0 ; 0)}, \quad \mathscr{C}_{\{0 \leq x \leq y\}, \infty}^{-1+\delta-\epsilon} \subset \mathscr{T}_{\{0 \leq x \leq y\}, \infty}^{-1+\delta-\epsilon,(0 ; 0)},
$$

justify the case $k=0$ of the following induction hypothesis, where $f_{2,0}=$ $\left(\varphi_{2,0}, \psi_{2,0}\right)=0$ (we hope that the reader will not get confused by a slight clash of notation, as the decomposition in the equations below is unrelated to the decomposition $\left.\psi=\left(\psi_{1}, \psi_{2}\right)\right)$ :

$$
\begin{aligned}
& \varphi=\underbrace{\varphi_{1, k}}_{\in x^{\delta-1} \mathscr{A}_{\{x=0\}}^{\delta}+x^{\delta-1} y \mathscr{A}_{\{0 \leq x \leq y\}}^{\delta}}+\underbrace{\varphi_{3, k}}_{\mathscr{A}_{\{x=0\}, \dot{\oplus}_{i} x^{i \delta} \mathscr{F}_{\{0 \leq x \leq y\}, \infty}^{\delta \delta-1+\delta-\epsilon-i \delta}}^{\varphi_{2, k}} \in \mathscr{T}_{\{0 \leq x \leq y\}, \infty}^{k \delta-1+\delta-\epsilon,(0 ; 0)}},
\end{aligned}
$$

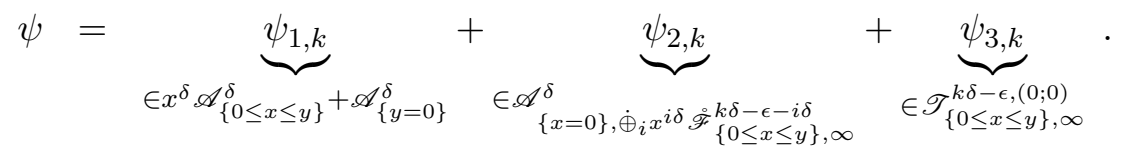

We note that

$$
x \varphi \in x^{\delta} \mathscr{A}_{\{x=0\}}^{\delta}+x^{\delta} y \mathscr{A}_{\{0 \leq x \leq y\}}^{\delta}+\mathscr{A}_{\{x=0\}, \dot{\oplus}_{i} x^{i \delta} \mathscr{F}_{\{0 \leq x \leq y\}, \infty}^{(k+1) \delta-\epsilon-i \delta}}^{\delta}+\mathscr{T}_{\{0 \leq x \leq y\}, \infty}^{(k+1) \delta-\epsilon,(0 ; 0)} .
$$

It follows that $\tilde{f}=\left(\psi_{1}, x \psi_{2}, x \varphi\right)$ belongs to the space

$$
\tilde{f} \in\left(\mathscr{A}_{\{0 \leq x \leq y\}}^{\delta}+\mathscr{A}_{\{x=0\}, \dot{\oplus}_{i} x^{i \delta} \mathscr{F}_{\{0 \leq x \leq y\}, \infty}^{\delta \delta-\epsilon-i \delta}}^{\delta}+\mathscr{T}_{\{0 \leq x \leq y\}, \infty}^{k \delta-\epsilon,(0 ; 0)}\right) .
$$

Note that our result is purely local, so without loss of generality we can take the domain $\mathscr{O}$ of the $v$-cordinates to be a closed coordinate ball. Lemma 3.13 below, with $\lambda=k \delta-\epsilon$, applied to $g=\tilde{f}$ gives

$x^{-p \delta} H\left(\cdot, x^{q \delta} \widetilde{f}\right) \in x^{(m q-p) \delta} \mathscr{A}_{\{0 \leq x \leq y\}}^{\delta}+\mathscr{A}_{\{x=0\}, \dot{\oplus}_{i} x^{i \delta} \mathscr{F}_{\{0 \leq x \leq y\}, \infty}^{(k+m q-p) \delta-i \delta}}^{\delta}+\mathscr{T}_{\{0 \leq x \leq y\}, \infty}^{(k+m q-p) \delta-\epsilon,(0 ; 0)}$.

We can repeat now the integration in (3.28), recovering (3.56) with $k=k+1$. This, together with (3.57) shows that (3.58) holds with $k$ replaced by $k+1$, hence (3.59) holds with $k$ replaced by $k+1$. An integration as in (3.29) gives now (3.55) with $k$ replaced by $k+1$, and the induction step is completed.

By an argument analogous to the one in the linear case, we conclude that

$$
\varphi \in x^{\delta-1} \mathscr{A}_{\{x=0\}}^{\delta}+x^{\delta-1} y \mathscr{A}_{\{0 \leq x \leq y\}}^{\delta}, \quad \psi \in x^{\delta} \mathscr{A}_{\{0 \leq x \leq y\}}^{\delta}+\mathscr{A}_{\{y=0\}}^{\delta}+\mathscr{A}_{\{x=0\}}^{\delta} .
$$

An analysis as in Remark 3.9 finishes the proof.

We finish this section with the lemma referred to above: 
LEMMA 3.13 Assume that $\mathscr{O}$ is convex, compact, with interior points. Let $q \in$ $\mathbb{N}^{*}, \mathbb{R} \ni \lambda \geq 0$, and let $H\left(x^{\mu}, w\right)$ be $\mathscr{A}_{\{0 \leq x \leq y\}}^{\delta}$-polyhomogeneous with respect to $x^{\mu}$ with a zero of order $m$ in $w$. If for all $\epsilon>0$ we have

$$
g \in\left(\mathscr{A}_{\{0 \leq x \leq y\}}^{\delta}+\mathscr{A}_{\{x=0\}, \dot{\oplus}_{i} x^{i \delta} \mathscr{F}_{\{0 \leq x \leq y\}, \infty}^{\lambda-i \delta}}^{\delta}+\mathscr{T}_{\{0 \leq x \leq y\}, \infty}^{\lambda-\epsilon,(0 ; 0)}\right),
$$

then it also holds, for all $\epsilon>0$,

$$
H\left(\cdot, x^{q \delta} g\right) \in x^{m q \delta}\left(\mathscr{A}_{\{0 \leq x \leq y\}}^{\delta}+\mathscr{A}_{\{x=0\}, \dot{\oplus}_{i} x^{i \delta} \mathscr{\mathscr { F }}_{\{0 \leq x \leq y\}, \infty}^{\lambda-i \delta}}^{\delta}+\mathscr{T}_{\{0 \leq x \leq y\}, \infty}^{\lambda-\epsilon,(0 ; 0)}\right) .
$$

If $\lambda>0$ and (3.60) holds with $\epsilon=0$, then (3.61) also holds with $\epsilon=0$.

Proof: This is a repetition of the proof of [11, Lemma 4.8], using an analogue of [11, Lemma A.5] in the $\mathscr{T}$-spaces. That last result is proved using the usual Moser inequality together with scaling, as follows: For each $\left(\bar{x}, \bar{v}^{A}, \bar{y}\right) \in \Omega$ we define a map $S_{\left(\bar{x}, \bar{v}^{A}, \bar{y}\right)}$ which maps a standard set of parameters $\mathscr{K} \times \mathscr{O}$ to a compact subset of $\Omega$, containing $\left(\bar{x}, \bar{v}^{A}, \bar{y}\right)$. By $\mathscr{K}$ we denote a "standard triangle":

$$
\mathscr{K}:=\{(s, t): 0 \leq s \leq t, 0 \leq t \leq 1\} .
$$

The maps $S_{\left(\bar{x}, \bar{v}^{A}, \bar{y}\right)}$ are defined as follows:

$$
(s, t, v) \mapsto(x, y, v)=\left\{\begin{array}{ll}
(\bar{x}+\varsigma s, \bar{y}+\varsigma t, v), \varsigma=\bar{x} & \text { for } \bar{y}<T \\
(\bar{x}+\varsigma(s-1), \bar{y}+\varsigma(t-1)), \varsigma=\bar{x} / 2 & \text { for } \bar{y} \geq T
\end{array} .\right.
$$

This definition guarantees that the $S$-image of $\mathscr{K} \times \mathscr{O}$ is contained in $\Omega$. We have

$$
h \in \mathscr{T}_{k}^{\alpha} \Longleftrightarrow \forall i+j+|\gamma| \leq k \quad\left|\partial_{v}^{\gamma} \partial_{x}^{i} \partial_{y}^{j} h\right| \leq C x^{\alpha-i-j} .
$$

There exists a smallest such $C$ which we denote by $\|h\|_{\mathscr{T}_{k}^{\alpha}}$. For $g \in \mathscr{T}_{k}^{\alpha}$ we estimate the norm of $H\left(\cdot, x^{q \delta} g\right)$ as follows:

$$
\begin{gathered}
\left\|H\left(\cdot, x^{q \delta} g\right)\right\|_{\mathscr{T}_{k}^{\alpha}(\bar{\Omega})} \leq \sum_{i+j+|\gamma| \leq k} \sup \left|x^{-\alpha+i+j} \partial_{v}^{\gamma} \partial_{x}^{i} \partial_{y}^{j} H\right|, \\
\partial_{v}^{\gamma} \partial_{x}^{i} \partial_{y}^{j} H=\left(\frac{1}{\varsigma}\right)^{i+j}\left(\partial_{v}^{\gamma} \partial_{s}^{i} \partial_{t}^{j} \tilde{H}\right) \circ S_{\left(\bar{x}, \bar{v}^{A}, \bar{y}\right)}^{-1},
\end{gathered}
$$

where $\tilde{H}=H \circ S_{\left(\bar{x}, \bar{v}^{A}, \bar{y}\right)}$. We use the interpolation inequality

$$
\|u\|_{C_{i}(\mathscr{K} \times \mathscr{O})} \leq\|u\|_{L^{\infty}(\mathscr{K} \times \mathscr{O})}^{1-i / k}\|u\|_{C_{k}(\mathscr{K} \times \mathscr{O})}^{i / k}, \quad i \leq k,
$$

and proceed as in [11, Proposition A.2] to estimate the derivatives of $\tilde{H}$. Let $z$ stand for $(s, t, v) \in \mathscr{K} \times \mathscr{O}$, then for $|\sigma| \leq k$ we have:

$$
\begin{aligned}
&\left|\partial^{\sigma} \tilde{H}\right| \leq C \mid \sum_{|\gamma|+\left|\sigma_{1}\right|+\cdots+\left|\sigma_{i}\right|=|\sigma|} \varsigma^{q \delta\left(\left|\sigma_{1}\right|+\cdots+\left|\sigma_{i}\right|\right)} \\
& \times \frac{\partial^{|\gamma|+i} \tilde{H}}{\partial z^{\gamma} \partial w^{i}} \partial^{\sigma_{1}}\left((1+s)^{q \delta} \tilde{g}\right) \cdots \partial^{\sigma_{i}}\left((1+s)^{q \delta} \tilde{g}\right) \mid \\
& \leq C \varsigma^{m q \delta} \sum_{\left|\sigma_{1}\right|+\cdots+\left|\sigma_{i}\right| \leq|\sigma|}\left|\partial^{\sigma_{1}}\left((1+s)^{q \delta} \tilde{g}\right)\right| \cdots\left|\partial^{\sigma_{i}}\left((1+s)^{q \delta} \tilde{g}\right)\right| \\
& \leq C \varsigma^{m q \delta}\left\|(1+s)^{q \delta} \tilde{g}\right\|_{L^{\infty}(\mathscr{K} \times \mathscr{O})}\left\|(1+s)^{q \delta} \tilde{g}\right\|_{C_{k}(\mathscr{K} \times \mathscr{O})},
\end{aligned}
$$


where $\tilde{g}=g \circ S_{\left(\bar{x}, \bar{v}^{A}, \bar{y}\right)}$. Let us note that $\bar{x}$ and $\varsigma$ are of the same order, in fact $\frac{\bar{x}}{\varsigma}$ equals 1 or 2 . Using this and the relationship between $g$ and $\tilde{g}$ we conclude that

$$
\left\|(1+s)^{q \delta} \tilde{g}\right\|_{C_{k}(\mathscr{K} \times \mathscr{O})} \leq C\|g\|_{\mathscr{T}_{k}^{\alpha}(\bar{\Omega})} \varsigma^{\alpha} .
$$

Putting all the estimates together we arrive at

$$
\left\|x^{-m q \delta} H\left(\cdot, x^{q \delta} g\right)\right\|_{\mathscr{T}_{k}^{\alpha}(\bar{\Omega})} \leq C\|g\|_{\mathscr{T}_{k}^{\alpha}(\bar{\Omega})},
$$

which is what we need to proceed with the proof of the Lemma. The point in all the estimates is that $C$ depends only on $q \delta, k$ and $\|g\|_{L^{\infty}}$ and does not depend on $\left(\bar{x}, \bar{v}^{A}, \bar{y}\right)$.

If $g \notin L^{\infty}(\bar{\Omega})$ we can use $\hat{g}_{\epsilon}:=x^{\epsilon} g \in L^{\infty}(\bar{\Omega})$, hence the epsilons in Lemma 3.13 .

\section{Acknowledgements}

We would like to acknowledge support by a Polish Research Committee grant 2 P03B 073 24, by the Schrödginer Institute, Vienna, and by the PAN-CNRS exchange programme. Sz. Ł. was also supported in part by a scholarship from the Foundation for Polish Science.

\section{A Function spaces, auxilliary results}

\section{A.1 $\mathscr{C}$-spaces}

For $k \in \mathbb{N}$ we denote by $C_{k}(\Omega)$ the set of all functions which are $k$ times continuously differentiable on $\Omega$. We denote by $C_{k}(\bar{\Omega})$ the set of $C_{k}(\Omega)$-functions which can be extended by continuity to $C_{k}$ functions defined in an open neighborhood of $\Omega$.

Let $F$ be a space of functions, we shall say that $f \in x^{\alpha} y^{\beta} F$ if $x^{-\alpha} y^{-\beta} f \in F$.

We need to introduce various families of function spaces with controlled singular behavior at $\{x=0\}$, or $\{y=0\}$, or $\{x=y=0\}$. The domains we will consider will always be subsets of the set $0 \leq x \leq y \leq y_{0}$ for some $y_{0}<\infty$. For $k \in \mathbb{N}$ we define

$$
\begin{aligned}
\mathscr{C}_{\{x=0\}, k}^{\alpha}(\Omega) & =\left\{f: \forall i, j \in \mathbb{N}, \beta \in \mathbb{N}^{r}, i+j+|\beta| \leq k, \sup _{\Omega}\left|x^{-\alpha} \partial_{v}^{\beta}\left[\partial_{y}\right]^{i}\left[x \partial_{x}\right]^{j} f\right|<\infty\right\}, \\
\mathscr{C}_{\{y=0\}, k}^{\sigma}(\Omega) & =\left\{f: \forall i, j \in \mathbb{N}, \beta \in \mathbb{N}^{r}, i+j+|\beta| \leq k, \sup _{\Omega}\left|y^{-\sigma} \partial_{v}^{\beta}\left[y \partial_{y}\right]^{i}\left[\partial_{x}\right]^{j} f\right|<\infty\right\}, \\
\mathscr{C}_{\{0 \leq x \leq y\}, k}^{\alpha}(\Omega) & =\left\{f: \forall i, j \in \mathbb{N}, \beta \in \mathbb{N}^{r}, i+j+|\beta| \leq k, \sup _{\Omega}\left|x^{-\alpha} \partial_{v}^{\beta}\left[y \partial_{y}\right]^{i}\left[x \partial_{x}\right]^{j} f\right|<\infty\right\}, \\
\mathscr{C}_{\{0 \leq x \leq y\}, k}^{\alpha, \sigma}(\Omega) & =\left\{f: \forall i, j \in \mathbb{N}, \beta \in \mathbb{N}^{r}, i+j+|\beta| \leq k, \sup _{\Omega}\left|x^{-\alpha} y^{-\sigma} \partial_{v}^{\beta}\left[y \partial_{y}\right]^{i}\left[x \partial_{x}\right]^{j} f\right|<\infty\right\} .
\end{aligned}
$$

The spaces $\mathscr{C}_{\{x=0\}, k}^{\alpha}$ here correspond to the spaces $\mathscr{C}_{k}^{\alpha}$ of [11].

We shall write

$$
\mathscr{C}_{\{0 \leq x \leq y\}, \infty}^{\alpha}=\cap_{k \in \mathbb{N}} \mathscr{C}_{\{0 \leq x \leq y\}, k}^{\alpha}
$$


similarly for $C_{\infty}(\Omega), \mathscr{C}_{\{x=0\}, \infty}^{\alpha}$, etc.

We note the following:

Proposition A.1 $\forall \delta \geq 0$ we have $x^{\alpha} \mathscr{C}_{\{y=0\}, k}^{\beta} \subset \mathscr{C}_{\{0 \leq x \leq y\}, k}^{\alpha-\delta, \beta+\delta}$.

Proof: There exist constants $C_{\ell, \alpha}$ and $C$ such that, for $0 \leq x \leq y$ and for $f \in \mathscr{C}_{\{y=0\}, k}^{\beta}$ we have

$$
\begin{aligned}
\left(x \partial_{x}\right)^{i}\left(y \partial_{y}\right)^{j} \partial_{v}^{\beta}\left(x^{\alpha} f\right) & =x^{\alpha} \sum_{\ell=0}^{i} C_{\ell, \alpha}\left(x \partial_{x}\right)^{\ell}\left(y \partial_{y}\right)^{j} \partial_{v}^{\beta} f \\
& \leq C x^{\alpha} y^{\beta}=C x^{\alpha-\delta} x^{\delta} y^{\beta} \leq C x^{\alpha-\delta} y^{\beta+\delta}
\end{aligned}
$$

\section{A.2 Polyhomogeneous functions ( $\mathscr{A}$-spaces $)$}

A function $f \in C_{\infty}(\Omega)$ will be said to be polyhomogeneous at $\{x=y=0\}$ if there exist integers $N_{i}$, real numbers $n_{i}, \hat{n}_{i}$, and functions $f_{i j \ell} \in C_{\infty}(\bar{\Omega})$ with the property that

$$
\begin{aligned}
& \forall m \in \mathbb{N} \quad \exists N(m) \text { such that } \\
& \quad f-\sum_{i=0}^{N(m)} \sum_{j, \ell=0}^{N_{i}} f_{i j \ell} y^{\hat{n}_{i}} x^{n_{i}} \ln ^{j} y \ln ^{\ell} x \in C_{m}(\bar{\Omega}) .
\end{aligned}
$$

We then write $f \in \mathscr{A}_{\{0 \leq x \leq y\}}$. To avoid repetitions of terms with identical powers in (A.1) it is convenient to impose $\left(n_{i}, \hat{n}_{i}\right) \neq\left(n_{j}, \hat{n}_{j}\right)$ for $i \neq j$, and we will always assume that this condition is satisfied.

We write

$$
f \in \mathscr{A}_{\{0 \leq x \leq y\}}^{\delta} \text { when } \delta \in 1 / \mathbb{N}^{*} \text { and }\left\{n_{i}\right\} \subset \delta \mathbb{N},\left\{\hat{n}_{i}\right\} \subset \delta \mathbb{Z}, \quad \hat{n}_{i} \geq-n_{i} .
$$

The last inequality (recall that $0 \leq x \leq y$ ) guarantees that functions in $\mathscr{A}_{\{0 \leq x \leq y\}}^{\delta}$ are estimated by $C\left(1+|\overline{\ln } x|^{N}\right)$ for some $N \in \mathbb{N}$, and that they are bounded up to a finite number of logarithmic terms. The need for negative powers of $y$ arises from the requirement of invariance of $\mathscr{A}_{\{0 \leq x \leq y\}}^{\delta}$ under $x \partial_{y}$. As an example, consider the function $\ln y \in \mathscr{A}_{\{0 \leq x \leq y\}}^{\delta}$ for any $\delta$, then $x \partial_{y} \ln y=x / y$. This exhibits the necessity of negative powers of $y$, appearing however in a way consistent with the inequalities in (A.2).

Similarly we shall write $f \in \mathscr{A}_{\{x=0\}}$ if (A.1) holds with $\hat{n}_{i}=0$ for all $i$, and with no non-trivial powers of $\ln y$, with the obvious definitions for $\mathscr{A}_{\{x=0\}}^{\delta}$, $\mathscr{A}_{\{y=0\}}$, etc.

The following observation will be used repeatedly:

Proposition A.2 1. We have the inclusion

$$
\mathscr{A}_{\{0 \leq x \leq y\}}^{\delta} \cap L^{\infty} \subset \mathscr{C}_{\{0 \leq x \leq y\}, \infty}^{0} .
$$


It follows that for any $\epsilon>0$ we have $\mathscr{A}_{\{0 \leq x \leq y\}}^{\delta} \subset \mathscr{C}_{\{0 \leq x \leq y\}, \infty}^{0-\epsilon}$.

2. Similarly

$$
\mathscr{A}_{\{x=0\}}^{\delta} \cap L^{\infty} \subset \mathscr{C}_{\{x=0\}, \infty}^{0},
$$

and for any $\epsilon>0$ we have $\mathscr{A}_{\{x=0\}}^{\delta} \subset \mathscr{C}_{\{x=0\}, \infty}^{0-\epsilon}$.

Proof: 1. The last statement is obvious given the previous ones. It remains to show that for all $k \in \mathbb{N}$ we have $\mathscr{A}_{\{0 \leq x \leq y\}}^{\delta} \cap L^{\infty} \subset \mathscr{C}_{\{0 \leq x \leq y\}, k}^{0}$. But clearly each term in the sum in (A.1) with $m=k$, as well as $f$ minus the whole sum, is in $\mathscr{C}_{\{0 \leq x \leq y\}, k}^{0}$, whence the result. The proof of point 2 . is identical.

We shall need the following characterisation of the space of polyhomogeneous functions:

Proposition A.3 $f \in \mathscr{A}_{\{x=0\}}$ if and only if for every $m \in \mathbb{N}$ there exist $N(m), N_{i}(m), n_{i}(m)$ and functions $f_{i j} \in C_{m}(\bar{\Omega})$ such that

$$
f-\sum_{i=0}^{N(m)} \sum_{j=0}^{N_{i}(m)} f_{i j} x^{n_{i}(m)} \ln ^{j} x \in C_{m}(\bar{\Omega}),
$$

with a similar property for $\mathscr{A}_{\{x=0\}}^{\delta}, \mathscr{A}_{\{0 \leq x \leq y\}}$, etc.

Proof: The direct implication is obvious, therefore we only need to prove the reverse one. We start by noting that in (A.3) we can choose the $f_{i j}$ 's to be independent of $x$. Indeed, if some $f_{i j}$ depends on $x$ one can Taylor-expand it with respect to $x=0$ :

$$
f_{i j}\left(x, v^{A}, y\right)=\sum_{\ell=0}^{n} \frac{1}{\ell !} \frac{\partial^{\ell} f_{i j}\left(0, v^{A}, y\right)}{\partial x^{\ell}} x^{\ell}+r .
$$

The coefficients of the expansion are in $C_{m-n}(\bar{\Omega})$. Therefore

$$
f_{i j} x^{n_{i}(m)} \ln ^{j} x=\sum_{\ell=0}^{n} \tilde{f}_{i j \ell}\left(v^{A}, y\right) x^{\ell+n_{i}(m)} \ln ^{j} x+x^{n_{i}(m)} \ln ^{j} x r .
$$

The last term is in $C_{k}(\bar{\Omega})$ with $k$ arbitrary large, provided one started with $m$ large enough. Similarly, one can arrange things so that the $C_{m}(\bar{\Omega})$ term in (A.3) is $o\left(x^{m}\right)$. Hence for every $m$ there exist $N(m), N_{i}(m), n_{i}(m)$ and $f_{i j}\left(v^{A}, y\right)$ such that

$$
f=\sum_{i=0}^{N(m)} \sum_{j=0}^{N_{i}(m)} f_{i j} x^{n_{i}(m)} \ln ^{j} x+r_{m}
$$

with $r_{m} \in C_{m}(\bar{\Omega})$ and $r_{m}=o\left(x^{m}\right)$. If necessary we rearrange the $n_{i}$ 's increasingly, and we also assume that $f_{i j}$ 's do not vanish identically.

The next step in the proof is to show that the $f_{i j}$ 's, defined as in (A.4), do not depend upon $m$, i.e., if one writes (A.4) with some $m^{\prime}$ then the $f_{i j}$ 's corresponding to $n_{i}<\min \left\{m, m^{\prime}\right\}$ will be equal. This is proved by comparing 
the expansions order by order, starting with the smallest $n_{i}$ and largest $j$. For example:

$f=\sum_{j=0}^{N_{0}(m)} f_{0 j} x^{n_{0}(m)} \ln ^{j} x+o\left(x^{n_{0}(m)+\epsilon}\right)=\sum_{j=0}^{N_{0}\left(m^{\prime}\right)} f_{0 j}^{\prime} x^{n_{0}\left(m^{\prime}\right)} \ln ^{j} x+o\left(x^{n_{0}\left(m^{\prime}\right)+\epsilon}\right)$.

Dividing this equation by $x^{n_{0}(m)} \ln ^{N_{0}(m)} x$ and taking the limit $x \rightarrow 0$ one gets $n_{0}(m) \leq n_{0}\left(m^{\prime}\right)$, similarly dividing by $x^{n_{0}\left(m^{\prime}\right)} \ln ^{N_{0}\left(m^{\prime}\right)} x$ one gets $n_{0}(m) \geq$ $n_{0}\left(m^{\prime}\right)$, hence $n_{0}(m)=n_{0}\left(m^{\prime}\right), N_{0}(m)=N_{0}\left(m^{\prime}\right)$ and $f_{0 N_{0}}=f_{0 N_{0}}^{\prime}$. Once the uniqueness of $N, N_{i}, n_{i}$ and $f_{i j}$ is established, the smoothness of $f_{i j}$ follows. This proves that $f \in \mathscr{A}_{\{x=0\}}$. The result in $\mathscr{A}_{\{x=0\}}^{\delta}$ follows as the property $n_{i} \in \delta \mathbb{N}$ is clearly preserved by the above procedure. The result for $\mathscr{A}_{\{0 \leq x \leq y\}}$ is proved similarly, using Taylor expansions both in $x$ and $y$.

The space denoted by $\mathscr{A}_{\{x=0\}}^{\delta}$ here coincides with the space $\mathscr{A}_{\infty}^{\delta}$ of [11]:

Proposition A.4 $f \in \mathscr{A}_{\{x=0\}}$ if and only if for all $\alpha \in \mathbb{R}$ there exists $\hat{N}(\alpha)$ and functions $f_{i \ell} \in C_{\infty}(\bar{\Omega})$ such that

$$
f-\sum_{i=0}^{\hat{N}(\alpha)} \sum_{\ell=0}^{N_{i}} f_{i \ell} x^{n_{i}} \ln ^{\ell} x \in \mathscr{C}_{\{x=0\}, \infty}^{\alpha} .
$$

This implies $\mathscr{A}_{\{x=0\}}^{\delta}=\mathscr{A}_{\infty}^{\delta}$.

Proof: Let $f$ satisfy the condition in the right member of the equivalence above, then the property $f \in \mathscr{A}_{\{x=0\}}$ follows from the inclusion $\mathscr{C}_{\{x=0\}, \infty}^{\alpha} \subset$ $C_{k}(\bar{\Omega})$ for $k<\alpha$. Reciprocally, suppose that there exist integers $N_{i}$, real numbers $n_{i}$, and functions $f_{i \ell} \in C_{\infty}(\bar{\Omega})$ such that for all $m \in \mathbb{N}$ there exists $N(m)$ such that

$$
f-\sum_{i=0}^{N(m)} \sum_{\ell=0}^{N_{i}} f_{i \ell} x^{n_{i}} \ln ^{\ell} x=: r_{m} \in C_{m}(\bar{\Omega}) .
$$

Replacing $f_{i l}$ by $x^{k} f_{i l}^{\prime}$ if necessary, without loss of generality we may assume that $\left.f_{i l}\right|_{x=0} \not \equiv 0$ for all $i, \ell \in \mathbb{N}$. From what has been said it should be clear that the set $\left\{n_{i} \leq 0\right\}$ is finite. It suffices to prove the result for

$$
f-\sum_{n_{i} \leq 0} \sum_{\ell=0}^{N_{i}} f_{i \ell} x^{n_{i}} \ln ^{\ell} x
$$

and thus without loss of generality we may assume that $f \in L^{\infty}$. By Proposition A.2 the left-hand side of the defining equality (A.6) is then in $\mathscr{C}_{\{x=0\}, \infty}^{0}$, thus $r_{m} \in \mathscr{C}_{\{x=0\}, \infty}^{0}$.

It follows from the definition that $\left.f\right|_{x=0} \in C_{\infty}$, which clearly implies that $\left.r_{m}\right|_{x=0} \in C_{\infty}$. One similarly shows that $\left.\left(\partial_{x}^{i} r_{m}\right)\right|_{x=0} \in C_{\infty}$ for $0 \leq i \leq m$. Let $r_{m}^{\prime}$ be defined as $r_{m}$ minus its Taylor series in $x$ of order $m-1$, then we still have $r_{m}^{\prime} \in \mathscr{C}_{\{x=0\}, \infty}^{0}$, and $r_{m}^{\prime}=O\left(x^{m}\right)$, thus

$$
r_{m}^{\prime} \in \mathscr{C}_{\{x=0\}, \infty}^{0} \cap \mathscr{C}_{\{x=0\}, 0}^{m} .
$$


Redefining the $f_{i \ell}$ 's, (A.6) still holds with $r_{m}$ replaced by $r_{m}^{\prime}$.

Let $m^{\prime}>m$, we then have

$$
r_{m}^{\prime}-\sum_{i=N(m)+1}^{N\left(m^{\prime}\right)} \sum_{\ell=0}^{N_{i}} f_{i \ell} x^{n_{i}} \ln ^{\ell} x=r_{m^{\prime}}^{\prime} \in \mathscr{C}_{\{x=0\}, \infty}^{0} \cap \mathscr{C}_{\{x=0\}, 0}^{m^{\prime}},
$$

with each term in the sum being $O\left(x^{m}\right)$ (otherwise $r_{m^{\prime}}^{\prime}$ wouldn't be $O\left(x^{m^{\prime}}\right)$ ). Recall the usual interpolation inequality [14], for $0<k<\ell$,

$$
\|f\|_{C_{k}} \leq C(k, \ell)\|f\|_{C_{0}}^{1-\frac{k}{\ell}}\|f\|_{C_{\ell}}^{\frac{k}{\ell}} ;
$$

its weighted equivalent reads (compare the proof of [11, Lemma A.4])

$$
\left\|r_{m^{\prime}}^{\prime}\right\|_{\mathscr{C}_{\{x=0\}, k}^{\left(1-\frac{k}{\ell}\right) m^{\prime}}} \leq C^{\prime}(k, \ell)\left\|r_{m^{\prime}}^{\prime}\right\|_{\mathscr{C}_{\{x=0\}, 0}^{m^{\prime}}}^{1-\frac{k}{\ell}}\left\|r_{m^{\prime}}^{\prime}\right\|_{\mathscr{C}_{\{x=0\}, \ell}^{0}}^{\frac{k}{\ell}}
$$

Given $k \in \mathbb{N}$ we choose $\ell=2 k, m^{\prime}=2 m$, leading to

$$
r_{m}^{\prime}-\sum_{i=N(m)+1}^{N\left(m^{\prime}\right)} \sum_{\ell=0}^{N_{i}} f_{i \ell} x^{n_{i}} \ln ^{\ell} x \in \mathscr{C}_{\{x=0\}, k}^{m} \quad \Longrightarrow \quad r_{m}^{\prime} \in \mathscr{C}_{\{x=0\}, k}^{m} .
$$

Since $k$ is arbitrary, we find that

$$
r_{m}^{\prime} \in \mathscr{C}_{\{x=0\}, \infty}^{m}
$$

and our claim follows.

Let $F$ be a space of functions on $\Omega$ such that $F \subset C_{\infty}(\Omega)$. We shall say that $f \in \mathscr{A}_{\{x=0\}, F}^{\delta}$ if for any $k \in \mathbb{N}$ there exists $N(k)$ and functions $\varphi_{i j} \in F$ such that

$$
f-\sum_{i, j=0}^{N(k)} \varphi_{i j} x^{\delta i} \ln ^{j} x \in C_{k}(\bar{\Omega}) .
$$

The spaces $\mathscr{A}_{\{0 \leq x \leq y\}, F}^{\delta}$ are defined in a similar way; for example we have the identity

$$
\mathscr{A}_{\{0 \leq x \leq y\}}^{\delta}=\mathscr{A}_{\{0 \leq x \leq y\}, C_{\infty}(\bar{\Omega})}^{\delta} .
$$

In this notation it holds that

$$
\mathscr{A}_{\{0 \leq x \leq y\}}^{\delta}=\mathscr{A}_{\{x=0\}, \mathscr{A}_{\{y=0\}}^{\delta}}^{\delta} .
$$

We will need the following characterisation of functions which are polyhomogeneous up to lower order terms. To avoid annoying special cases involving logarithms we assume $\sigma \notin \mathbb{N}$, though the proof gives also a corresponding statement in this case: 
Proposition A.5 Suppose that $\sigma \notin \mathbb{N}$, let

$$
\left.f\right|_{\mathscr{S}} \in x^{\beta} \mathscr{A}_{\{0 \leq x \leq y\}}, \quad f \in x^{\beta} \mathscr{A}_{\{0 \leq x \leq y\}}^{\delta}+y^{\beta} \mathscr{A}_{\{0 \leq x \leq y\}}^{\delta}+\mathscr{C}_{\{0 \leq x \leq y\}, k}^{\sigma},
$$

and assume that for all $i, j$ satisfying $i+j \leq k+1$ there exists

$$
g_{i, j} \in x^{\beta} \mathscr{A}_{\{0 \leq x \leq y\}}^{\delta}+y^{\beta} \mathscr{A}_{\{0 \leq x \leq y\}}^{\delta}
$$

such that for every multi-index $\gamma$ for which $i+j+|\gamma|=k+1$ we have

$$
\left|\left(x \partial_{x}\right)^{i}\left(y \partial_{y}\right)^{j} \partial_{v}^{\gamma}\left(f-g_{i, j}\right)\right| \leq C x^{\sigma} .
$$

Then

$$
f \in x^{\beta} \mathscr{A}_{\{0 \leq x \leq y\}}^{\delta}+y^{\beta} \mathscr{A}_{\{0 \leq x \leq y\}}^{\delta}+\mathscr{C}_{\{0 \leq x \leq y\}, k+1}^{\sigma} .
$$

Proof: For $\sigma<\beta$ and, simultaneously, $\sigma<0$ there is nothing to prove, as then the first two spaces are included in the third one, and the claimed decomposition of $f$ is uninteresting, and can be done in many different ways. We therefore assume that at least one of those inequalities is violated, and we proceed by induction on $k$. Suppose we know that the property

$$
\partial_{A} f, y \partial_{y} f, x \partial_{x} f \in x^{\beta} \mathscr{A}_{\{0 \leq x \leq y\}}^{\delta}+y^{\beta} \mathscr{A}_{\{0 \leq x \leq y\}}^{\delta}+\mathscr{C}_{\{0 \leq x \leq y\}, k}^{\sigma}
$$

implies (A.9). Then Proposition A.5 is also established for $k=0$. Next, applying the already established case $k=0$ of the proposition to $\left(x \partial_{x}\right)^{\ell-1} f$ we find that

$$
\left(x \partial_{x}\right)^{\ell-1} f \in x^{\beta} \mathscr{A}_{\{0 \leq x \leq y\}}^{\delta}+y^{\beta} \mathscr{A}_{\{0 \leq x \leq y\}}^{\delta}+\mathscr{C}_{\{0 \leq x \leq y\}, 1}^{\sigma} .
$$

Similarly, applying the case $k=0$ of the current proposition to $y \partial_{y}\left(x \partial_{x}\right)^{\ell-2} f$ and $\partial_{A}\left(x \partial_{x}\right)^{\ell-2} f$ we find that

$$
\begin{aligned}
& y \partial_{y}\left(x \partial_{x}\right)^{\ell-2} f \in x^{\beta} \mathscr{A}_{\{0 \leq x \leq y\}}^{\delta}+y^{\beta} \mathscr{A}_{\{0 \leq x \leq y\}}^{\delta}+\mathscr{C}_{\{0 \leq x \leq y\}, 1}^{\sigma}, \\
& \partial_{A}\left(x \partial_{x}\right)^{\ell-2} f \in x^{\beta} \mathscr{A}_{\{0 \leq x \leq y\}}^{\delta}+y^{\beta} \mathscr{A}_{\{0 \leq x \leq y\}}^{\delta}+\mathscr{C}_{\{0 \leq x \leq y\}, 1}^{\sigma} .
\end{aligned}
$$

The implication $(\mathrm{A} .10) \Rightarrow(\mathrm{A} .9)$ gives then

$$
\left(x \partial_{x}\right)^{\ell-2} f \in x^{\beta} \mathscr{A}_{\{0 \leq x \leq y\}}^{\delta}+y^{\beta} \mathscr{A}_{\{0 \leq x \leq y\}}^{\delta}+\mathscr{C}_{\{0 \leq x \leq y\}, 2}^{\sigma} .
$$

Taking $\ell=2$ establishes Proposition A.5 with $k=1$. Continuing in this way, Proposition A.5 follows for all $k \in \mathbb{N}$.

It remains to establish the implication $($ A.10) $\Rightarrow($ A.9). Writing

$$
\partial_{x} f=\underbrace{f_{x, k, \mathrm{phg}}}_{\in x^{\beta-1} \mathscr{A}_{\{0 \leq x \leq y\}}^{\delta}+x^{-1} y^{\beta} \mathscr{A}_{\{0 \leq x \leq y\}}^{\delta}}+\underbrace{f_{x, k, \sigma}}_{\in \mathscr{C}_{\{0 \leq x \leq y\}, k}^{\sigma-1}},
$$

we have

$$
f\left(x, v^{A}, y\right)=\underbrace{f\left(y, v^{A}, y\right)+\int_{y}^{x} f_{x, k, \mathrm{phg}}\left(s, v^{A}, y\right) d s}_{=: f_{k+1, \mathrm{phg}} \in x^{\beta} \mathscr{A}_{\{0 \leq x \leq y\}}^{\delta}+y^{\beta} \mathscr{A}_{\{0 \leq x \leq y\}}^{\delta}}+\underbrace{\int_{y}^{x} f_{x, k, \sigma}\left(s, v^{A}, y\right) d s}_{=: f_{k+1, \sigma}} .
$$


We want to show that $f_{k+1, \sigma} \in \mathscr{C}_{\{0 \leq x \leq y\}, k+1}^{\sigma}$; equivalently:

$$
\forall \gamma, i+j+|\gamma| \leq k+1 \quad\left|\partial_{v}^{\gamma}\left(\partial_{x}\right)^{i}\left(\partial_{y}\right)^{j} f_{k+1, \sigma}\right| \leq C x^{\sigma-i} y^{-j}
$$

The inequality is clear if $i \geq 1$ or if $|\gamma|+j<k+1$, by differentiating the integral defining $f_{k+1, \sigma}$. Suppose thus that $i=0$ and $|\gamma|+j=k+1$. Recall that, by hypothesis, we have

$$
\partial_{v}^{\gamma}\left(y \partial_{y}\right)^{j} f=\underbrace{\partial_{v}^{\gamma} f_{j, y, k+1, \mathrm{phg}}}_{\in x^{\beta} \mathscr{A}_{\{0 \leq x \leq y\}}^{\delta}+y^{\beta} \mathscr{A}_{\{0 \leq x \leq y\}}^{\delta}}+O\left(x^{\sigma}\right),
$$

and comparing with (A.11),

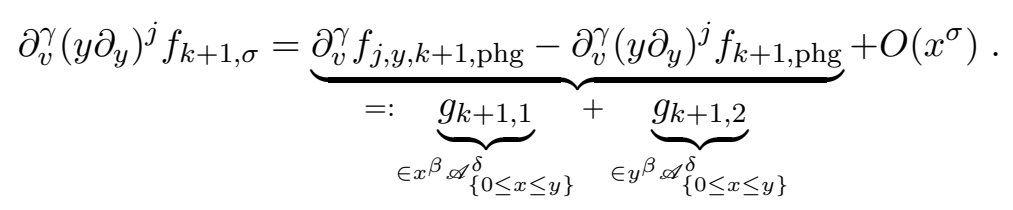

Recall, now, that we only have to consider the cases $\sigma \geq \beta$, or $\sigma \geq 0$, or both. For $y>0$ consider the collected polyhomogeneous terms in (A.12), they can be written as a finite sum

$$
\begin{aligned}
g_{k+1,1}\left(x, y, v^{A}\right) & =\sum_{\beta+n \delta \leq \sigma} \sum_{m}^{N_{n}} f_{m n}\left(y, v^{A}\right) x^{\beta+n \delta} \ln ^{m} x+r_{k+1,1}\left(x, y, v^{A}\right) \\
g_{k+1,2}\left(x, y, v^{A}\right) & =\sum_{n \delta \leq \sigma} \sum_{m}^{N_{n}^{\prime}} f_{m n}^{\prime}\left(y, v^{A}\right) x^{n \delta} \ln ^{m} x+r_{k+1,2}\left(x, y, v^{A}\right)
\end{aligned}
$$

with functions $f_{n m}$ and $f_{n m}^{\prime}$ which are smooth in both variables (as long as $y>0$ ), and with the remainders being $O\left(x^{\sigma}\right)$. If $\beta \in-\mathbb{N}$ we absorb the second sum in the first one; similarly if $\beta \in \mathbb{N}$ we absorb the first sum in the second one. This shows that without loss of generality we can always assume that all powers of $x$ appearing in the sums above are now pairwise distinct. Suppose that $f_{m n} \not \equiv 0$ or $f_{m n}^{\prime} \not \equiv 0$ for some couple $m n$, then $f_{k+1, \sigma}$ wouldn't be $O\left(x^{\sigma}\right)$ for all $\left(y, v^{A}\right)$ 's by integration of (A.12) in $y$ or in $v$. It follows that

$$
g_{k+1,1}+g_{k+1,2}=r_{k+1,1}+r_{k+1,2}=O\left(x^{\sigma}\right),
$$

which establishes our claim.

\section{A.3 $\mathscr{F}-$ and $\mathscr{T}$-spaces}

For $\alpha \in \mathbb{R}$ and $k \in \mathbb{N}$ we set

$$
\begin{aligned}
& \mathscr{F}_{\{0 \leq x \leq y\}, k}^{\alpha}=\{f|\forall 0 \leq i+j+| \gamma \mid \leq k \exists N: \\
&\left|\partial_{x}^{i} \partial_{y}^{j} \partial_{v}^{\gamma} f\right| \leq\left\{\begin{array}{ll}
C y^{\alpha-i-j}(1+|\ln y|)^{N} & \text { if } \alpha-i-j \geq 0 \\
C x^{\alpha-i-j}(1+|\ln x|)^{N} & \text { if } \alpha-i-j<0
\end{array}\right\} .
\end{aligned}
$$


We will also need a version of the $\mathscr{F}$-spaces where the functions involved are "almost independent of $x$ when $\alpha$ is large", in the following sense:

$$
\begin{aligned}
& \stackrel{\circ}{\alpha} \mathscr{F}_{\{0 \leq x \leq y\}, k}=\{f|\forall 0 \leq i+j+| \gamma \mid \leq k \exists N: \\
&\left|\partial_{x}^{i} \partial_{y}^{j} \partial_{v}^{\gamma} f\right| \leq\left\{\begin{array}{ll}
C y^{\alpha-j}(1+|\ln y|)^{N} & \text { if } \alpha-j \geq 0, i=0 \\
C x^{\alpha-i-j}(1+|\ln x|)^{N} & \text { otherwise }
\end{array}\right\}
\end{aligned}
$$

Let $\alpha, \beta \in \mathbb{R}, k \in \mathbb{N}$. To be able to estimate in terms of powers of $|\ln x|$ rather than $1+|\ln x|$ it is convenient to assume $0<y_{0}<1$. We say that $f \in \mathscr{T}_{\{0 \leq x \leq y\}, \infty}^{\alpha,(\beta ; k)}$ if for all $i, j, \gamma$ there exist constants $C>0$ and $N \in \mathbb{N}$ such that, for $0<x \leq y \leq y_{0}$ we have

$$
\left|\partial_{x}^{i} \partial_{y}^{j} \partial_{v}^{\gamma} f\right| \leq C\left(x^{\alpha+\beta-i-j}+x^{\alpha-i} y^{\beta-j}+x^{\alpha+\beta-i-k} y^{k-j}\right)\left|\ln ^{N} x\right| \text {. }
$$

We write $f \in \mathscr{T}_{\{0 \leq x \leq y\}, \infty}^{\alpha, \beta}$ for $f \in \mathscr{T}_{\{0 \leq x \leq y\}, \infty}^{\alpha,(\beta ; 0)}$, and we note that for $k=0$, or for $\beta=k$, the last term in (A.15) is not needed, e.g.:

$$
f \in \mathscr{T}_{\{0 \leq x \leq y\}, \infty}^{\alpha, \beta} \Longleftrightarrow\left|\partial_{x}^{i} \partial_{y}^{j} \partial_{v}^{\gamma} f\right| \leq C\left(x^{\alpha+\beta-i-j}+x^{\alpha-i} y^{\beta-j}\right)\left|\ln ^{N} x\right|
$$

Finally, for $\beta \leq 0$ the last term in (A.16) can be dropped altogether.

Strictly speaking, the only space out of the $\mathscr{T}_{\{0 \leq x \leq y\}, \infty}^{\alpha,(\beta ; k)}$ 's which is absolutely necessary in our proofs is the one with $k=\beta=0$. However, we have decided to include a short discussion of the other ones as well, as those spaces appear naturally in the problem at hand.

Let $\left\{F_{i}\right\}_{i \in \mathbb{N}}$ be any countable family of function spaces, we shall write

$$
\dot{\oplus}_{n} F_{n}=\left\{f: \exists N \in \mathbb{N}, f_{n} \in F_{n}, 0 \leq n \leq N, f=\sum_{n=0}^{N} f_{n}\right\} .
$$

The dot over the symbol $\oplus$ is meant to emphasise the fact that only finite linear combinations are considered.

For further use we note the following elementary properties:

Proposition A.6 1. If $f \in \mathscr{T}_{\{0 \leq x \leq y\}, \infty}^{\alpha,(\beta ; k)}$ then $\partial_{x} f \in \mathscr{T}_{\{0 \leq x \leq y\}, \infty}^{\alpha-1,(\beta ; k)}$ and $\partial_{y} f \in$ $\mathscr{T}_{\{0 \leq x \leq y\}, \infty}^{\alpha,(\beta-1 ; \max (k-1,0))}$

2. For $\alpha^{\prime} \geq \alpha$ and $\beta^{\prime} \geq \beta$ we have $\mathscr{T}_{\{0 \leq x \leq y\}, \infty}^{\alpha^{\prime},\left(\beta^{\prime} ; k\right)} \subset \mathscr{T}_{\{0 \leq x \leq y\}, \infty}^{\alpha,(\beta ; k)}$.

3. For $\sigma \geq 0$ we have $\mathscr{T}_{\{0 \leq x \leq y\}, \infty}^{\alpha+\sigma,(\beta ; k)} \subset \mathscr{T}_{\{0 \leq x \leq y\}, \infty}^{\alpha,(\beta+\sigma ; k)}$.

4. If $\mathbb{N} \ni \ell<\alpha$ and $\ell \leq k \leq \beta$ we have $\mathscr{T}_{\{0 \leq x \leq y\}, \infty}^{\alpha,(\beta ; k)} \subset \mathscr{C}_{\{y=0\}, \ell}^{\beta}$.

5. $\mathscr{C}_{\{x=0\}, \infty}^{\alpha} \subset \mathscr{C}_{\{0 \leq x \leq y\}, \infty}^{\alpha} \subset \mathscr{T}_{\{0 \leq x \leq y\}, \infty}^{\alpha,(0 ; k)}$, and $\mathscr{C}_{\{y=0\}, \infty}^{\beta} \subset \mathscr{T}_{\{0 \leq x \leq y\}, \infty}^{0,(\beta ; k)}$ for all $k$. 
6. If $f \in \mathscr{T}_{\{0 \leq x \leq y\}, \infty}^{\alpha,(\beta ; k)}$ and $g \in C^{\infty}$, then $f g \in \mathscr{T}_{\{0 \leq x \leq y\}, \infty}^{\alpha,(\beta ; k)}$.

7. If $g \in \mathscr{C}_{\{x=0\}, \infty}^{\alpha}$ and $h \in \mathscr{C}_{\{y=0\}, \infty}^{\beta}$ then $g h \in \mathscr{T}_{\{0 \leq x \leq y\}, \infty}^{\alpha,(\beta ; k)}$ for all $k$.

8. We have $x^{\sigma} \mathscr{T}_{\{0 \leq x \leq y\}, \infty}^{\alpha,(\beta ; k)}=\mathscr{T}_{\{0 \leq x \leq y\}, \infty}^{\alpha+\sigma,(\beta ; k)}$ for all $k \in \mathbb{N}$ and $\sigma \in \mathbb{R}$.

9. For $f \in \mathscr{T}_{\{0 \leq x \leq y\}, \infty}^{\alpha,(\beta ; k)}$ and $\ell \in \mathbb{N}$ we have $x^{\ell} f \in \mathscr{T}_{\{0 \leq x \leq y\}, \infty}^{\alpha,(\beta+\ell, k+\ell)}$ for all $k$.

Proof: Points 6-8 follow immediately from the formula

$$
\partial_{x}^{i} \partial_{y}^{j}(g h)=\sum_{\begin{array}{c}
r+s=i \\
m+n=j
\end{array}} C(r, s, m, n)\left(\partial_{x}^{r} \partial_{y}^{m} g\right)\left(\partial_{x}^{s} \partial_{y}^{n} h\right) .
$$

The remaining claims are direct consequences of the definition.

\section{A.4 Extensions of a class of functions}

Let $0 \leq \varphi \in C^{\infty}(\mathbb{R}), \operatorname{supp} \varphi \subset[-1 / 2,1 / 2], \int_{\mathbb{R}} \varphi(x) d x=1$. For $0<x \leq y \leq y_{0}$ we set

$$
\begin{aligned}
E[f](x, y, v) & :=\int_{0}^{\infty} \frac{\varphi\left(\frac{w-y}{x}\right)}{x} f(w, v) d w \\
& =\int_{y / 2}^{3 y / 2} \frac{\varphi\left(\frac{w-y}{x}\right)}{x} f(w, v) d w \\
& =\int_{-\infty}^{\infty} \frac{\varphi\left(\frac{w-y}{x}\right)}{x} f(w, v) d w \\
& =\int_{-\infty}^{\infty} \varphi(z) f(y+x z, v) d z \\
& =\int_{-1 / 2}^{1 / 2} \varphi(z) f(y+x z, v) d z
\end{aligned}
$$

(there is no need to know the values of $f$ for negative $w$ when using (A.18c) as $\varphi=0$ there; a similar comment applies to (A.18d)).

The results here are an adaptation to the problem at hand of [2, Section 3.3]. In the lemma that follows one can think of $\mu$ as belonging to $[0,1)$, but this restriction is not necessary for the result:

Lemma A.7 For $k \in \mathbb{N}$ and $\mu \in \mathbb{R}$ suppose that

$$
\left|\partial_{v}^{\gamma} \partial_{y}^{\ell} f\right| \leq C y^{k+\mu-\ell}(1+|\ln y|)^{N} \text { for } 0 \leq \ell \leq k,
$$

then

$$
E[f] \in y^{\mu} \mathscr{F}_{\{0 \leq x \leq y\}, \infty}^{k} .
$$

If moreover there exists $\lambda>0$ such that

$\left|\partial_{v}^{\gamma} \partial_{y}^{k} f(y, v)-\partial_{v}^{\gamma} \partial_{y}^{k} f\left(y^{\prime}, v\right)\right| \leq C y^{\mu-\lambda}(1+|\ln y|)^{N}\left|y-y^{\prime}\right|^{\lambda}$ for $\left|y^{\prime}-y\right| \leq y / 2$,

then we also have

$$
E[f](x, y, v) \in y^{\mu-\lambda} \mathscr{F}_{\{0 \leq x \leq y\}, \infty}^{k+\lambda} .
$$


Proof: The estimate (A.19) together with (A.18e) gives

$$
\begin{aligned}
0 \leq i+j \leq k \quad\left|\partial_{x}^{i} \partial_{y}^{j} \partial_{v}^{\gamma} E[f](x, y, v)\right| & =\left|\int_{-1 / 2}^{1 / 2} \varphi(z) \partial_{y}^{i+j} \partial_{v}^{\gamma} f(y+x z, v) z^{i} d z\right| \\
& \leq C y^{k+\mu-i-j}(1+|\ln y|)^{N} . \quad \text { (A.23) }
\end{aligned}
$$

On the other hand, if $i+j>k$ we write $i=i_{1}+i_{2}$ and $j=j_{1}+j_{2}$ with $i_{2}+j_{2}=k$, obtaining

$$
\begin{aligned}
\partial_{x}^{i} \partial_{y}^{j} \partial_{v}^{\gamma} E[f](x, y, v) & =\partial_{x}^{i_{1}} \partial_{y}^{j_{1}} \int_{-1 / 2}^{1 / 2} \underbrace{\varphi(z) z^{i_{2}}}_{=: \varphi_{i_{2}}(z)} \partial_{y}^{k} \partial_{v}^{\gamma} f(y+x z, v) d z \\
& =\partial_{x}^{i_{1}} \partial_{y}^{j_{1}} \int_{0}^{\infty} \frac{\varphi_{i_{2}\left(\frac{w-y}{x}\right)}}{x} \partial_{y}^{k} \partial_{v}^{\gamma} f(w, v) d w \\
& =x^{-i_{1}-j_{1}} \int_{0}^{\infty} \frac{\varphi_{i_{1}, i_{2}, j_{1}}\left(\frac{w-y}{x}\right)}{x} \partial_{y}^{k} \partial_{v}^{\gamma} f(w, v) d w
\end{aligned}
$$

where we have set

$$
\varphi_{i_{1}, i_{2}, j_{1}}\left(\frac{w-y}{x}\right):=x^{1+i_{1}+j_{1}} \partial_{x}^{i_{1}} \partial_{y}^{j_{1}}\left(\frac{\varphi_{i_{2}}\left(\frac{w-y}{x}\right)}{x}\right) .
$$

It follows that

$$
\begin{aligned}
\left|\partial_{x}^{i} \partial_{y}^{j} \partial_{v}^{\gamma} E[f](x, y, v)\right| & \leq C x^{-i_{1}-j_{1}} y^{\mu}(1+|\ln y|)^{N} \int_{0}^{\infty}\left|\frac{\varphi_{i_{1}, i_{2}, j_{1}}\left(\frac{w-y}{x}\right)}{x}\right| d w \\
& =C x^{-i_{1}-j_{1}} y^{\mu}(1+|\ln y|)^{N} \int_{-1 / 2}^{1 / 2}\left|\varphi_{i_{1}, i_{2}, j_{1}}(z)\right| d z \\
& \leq C C^{\prime} x^{-i_{1}-j_{1}} y^{\mu}(1+|\ln y|)^{N} \\
& =C C^{\prime} x^{k-i-j} y^{\mu}(1+|\ln y|)^{N}
\end{aligned}
$$

which establishes (A.20).

To prove (A.22), we start by noting that for $i_{1}+j_{1}>0$ we have

$$
\begin{aligned}
0 & =\partial_{x}^{i_{1}} \partial_{y}^{j_{1}} \int_{-\infty}^{\infty} \varphi_{i_{2}}(z) d z \\
& =\partial_{x}^{i_{1}} \partial_{y}^{j_{1}} \int_{0}^{\infty} \frac{\varphi_{i_{2}}\left(\frac{w-y}{x}\right)}{x} d w=\int_{0}^{\infty} \partial_{x}^{i_{1}} \partial_{y}^{j_{1}}\left(\frac{\varphi_{i_{2}}\left(\frac{w-y}{x}\right)}{x}\right) d w
\end{aligned}
$$

This allows us to write (compare (A.24))

$$
\begin{aligned}
\partial_{x}^{i} \partial_{y}^{j} \partial_{v}^{\gamma} E[f](x, y, v) & =\int_{0}^{\infty} \partial_{x}^{i_{1}} \partial_{y}^{j_{1}}\left(\frac{\varphi_{i_{2}}\left(\frac{w-y}{x}\right)}{x}\right)\left(\partial_{y}^{k} \partial_{v}^{\gamma} f(w, v)-\partial_{y}^{k} \partial_{v}^{\gamma} f(y, v)\right) d w \\
& =x^{-i_{1}-j_{1}} \int_{0}^{\infty} \frac{\varphi_{i_{1}, i_{2}, j_{1}}\left(\frac{w-y}{x}\right)}{x}\left(\partial_{y}^{k} \partial_{v}^{\gamma} f(w, v)-\partial_{y}^{k} \partial_{v}^{\gamma} f(y, v)\right) d w \\
& =x^{-i_{1}-j_{1}} \int_{\infty}^{\infty} \varphi_{i_{1}, i_{2}, j_{1}}(z) \underbrace{\left(\partial_{y}^{k} \partial_{v}^{\gamma} f(y+z x, v)-\partial_{y}^{k} \partial_{v}^{\gamma} f(y, v)\right)}_{\leq C y^{\mu-\lambda}(1+|\ln y|)^{N} x^{\lambda}} d z \\
& \leq C^{\prime} y^{\mu-\lambda}(1+|\ln y|)^{N} x^{k+\lambda-i-j},
\end{aligned}
$$


as desired.

We continue with

LEMMA A.8 Let $\mu \geq 0$ and for $0 \leq i \leq m$ let $f_{i}$ satisfy (A.19) with $k$ there replaced by $m-i$. There exists $h \in y^{\mu} \mathscr{F}_{\{0 \leq x \leq y\}, \infty}^{m}$ such that

$$
0 \leq i \leq\left. m \quad \partial_{x}^{i} h\right|_{x=0}=f_{i} .
$$

If the $f_{i}$ 's satisfy (A.21) with $k=m-i$ then $h \in y^{\mu-\lambda} \mathscr{F}_{\{0 \leq x \leq y\}, \infty}^{m+\lambda}$.

Proof: We start by considering the following set of functions, defined for $0 \leq i \leq m$,

$$
g_{i}(x, y, v)=\frac{x^{i}}{i !} E\left[f_{i}\right](x, y, v)
$$

It follows from Proposition A.7 that $g_{i} \in y^{\mu} x^{i} \mathscr{F}_{\{0 \leq x \leq y\}, \infty}^{m-i} \subset y^{\mu} \mathscr{F}_{\{0 \leq x \leq y\}, \infty}^{m}$, or $g_{i} \in y^{\mu-\lambda} x^{i} \mathscr{F}_{\{0 \leq x \leq y\}, \infty}^{m+\lambda-i} \subset y^{\mu-\lambda} \mathscr{F}_{\{0 \leq x \leq y\}, \infty}^{m+\lambda}$ if (A.22) holds. It also follows from (A.23) and from Lebesgue's differentiation theorem that for $0 \leq j \leq m$ the functions $\partial_{x}^{j} g_{i}$ extend by continuity to continuous functions on $\{x=0\}$, and that $\left.g_{i}\right|_{x=0}$ satisfies (A.19) with $k$ there equal to $m-i$, and with the modulus of Hölder continuity satisfying (A.22) if this condition was satisfied by the $f_{i}$ 's.

Those considerations imply that the following inductive scheme is well defined: we set $h_{0}=E\left[f_{0}\right]$ and

$$
h_{i+1}(x, y, v)=h_{i}(x, y, v)+\frac{x^{i}}{i !} E\left[f_{i}-\left.\left(\partial_{x}^{i} h_{i}\right)\right|_{x=0}\right]
$$

Then the function $h:=h_{m}$ satisfies (A.26), and belongs to the spaces claimed.

\section{A.5 Integral operators on $\mathscr{A}-$ and $\mathscr{C}$-spaces}

For $0 \leq x \leq y \leq y_{0}<\infty$ set

$$
\begin{aligned}
& I_{1}(f)\left(x, v^{A}, y\right)=\int_{x}^{y} f\left(s, v^{A}, y\right) d s, \\
& I_{2}(f)\left(x, v^{A}, y\right)=\int_{x}^{y} f\left(x, v^{A}, s\right) d s .
\end{aligned}
$$

In our arguments we will need to understand the action of $I_{1}$ and $I_{2}$ on various spaces defined above. We start with polyhomogeneous functions:

Proposition A.9

$$
\begin{aligned}
& \text { 1. Let } g \in x^{\beta} y^{\gamma} \mathscr{A}_{\{0 \leq x \leq y\}}^{\delta} \text {. Then } \\
& I_{1}(g) \in y^{\beta+\gamma+1} \mathscr{A}_{\{y=0\}}^{\delta}+x^{\beta+1} y^{\gamma} \mathscr{A}_{\{0 \leq x \leq y\}}^{\delta}, \\
& I_{2}(g) \in x^{\beta+\gamma+1} \mathscr{A}_{\{x=0\}}^{\delta}+x^{\beta} y^{\gamma+1} \mathscr{A}_{\{0 \leq x \leq y\}}^{\delta} .
\end{aligned}
$$

It follows in particular that $\mathscr{A}_{\{0 \leq x \leq y\}}$ is stable under both integrations above. 
2. Let $g \in x^{\beta} y^{\gamma} \mathscr{A}_{\{x=0\}}^{\delta}$. Then

$$
\begin{aligned}
& I_{1}(g) \in y^{\beta+\gamma+1} \mathscr{A}_{\{y=0\}}^{\delta}+x^{\beta+1} y^{\gamma} \mathscr{A}_{\{x=0\}}^{\delta}, \\
& I_{2}(g) \in x^{\beta+\gamma+1} \mathscr{A}_{\{x=0\}}^{\delta}+x^{\beta} y^{\gamma+1} \mathscr{A}_{\{x=0\}}^{\delta} .
\end{aligned}
$$

Proof: Let $f \in C_{\infty}(\bar{\Omega}), p \in \mathbb{R}, j \in \mathbb{N}$. We start by showing that for every $m \in \mathbb{N}$ there exist an integer $N$, sequences of numbers $k_{i} \in \mathbb{N}, \ell_{i} \in \mathbb{N}$, a sequence of smooth functions $f_{i}$ and a function $r_{m} \in C_{m}(\bar{\Omega})$ such that

$$
\int_{x}^{y} f\left(s, v^{A}, y\right) s^{p} \ln ^{j} s d s=\sum_{i=1}^{N} f_{i}\left(y^{p+k_{i}+1} \ln ^{\ell_{i}} y-x^{p+k_{i}+1} \ln ^{\ell_{i}} x\right)+r_{m} .
$$

Several integrations by parts in the integral $\int v^{\prime} u$ with $v^{\prime}=s^{p}$ and $u=\ln ^{j} s$ yield this formula when $\partial_{x} f=0$. The result for general $f$ is also obtained by integration by parts by taking $u=f$ and $v^{\prime}=s^{p} \ln ^{j} s$. Using the result already proved with $f=1$ one obtains a $v$ with a power of $s$ higher by one. Repeating the integration by parts a finite number of times one obtains a remainder term in $C_{m}(\bar{\Omega})$, and one concludes by Proposition A.3.

We continue with a study of the action of $I_{1}$ and $I_{2}$ on the $\mathscr{C}_{\{0 \leq x \leq y\}, k}^{\alpha, \sigma}$ spaces. Note that the action on the $\mathscr{C}_{\{0 \leq x \leq y\}, k}^{\alpha}$ spaces is obtained as a special case from

$$
\mathscr{C}_{\{0 \leq x \leq y\}, k}^{\alpha}=\mathscr{C}_{\{0 \leq x \leq y\}, k}^{\alpha, 0} .
$$

Lemma A.10 Let $\alpha, \sigma \in \mathbb{R}, k \in \mathbb{N} \cup\{\infty\}$,

1. If $f \in \mathscr{C}_{\{0 \leq x \leq y\}, k}^{\alpha, \sigma}, \alpha<-1$, then $I_{1}(f) \in \mathscr{C}_{\{0 \leq x \leq y\}, k}^{\alpha+1, \sigma}$.

2. If $f \in \mathscr{C}_{\{0 \leq x \leq y\}, k}^{\alpha, \sigma}, \alpha>-1$, then $I_{1}(f) \in \mathscr{C}_{\{y=0\}, k}^{\alpha+\sigma+1}+\mathscr{C}_{\{0 \leq x \leq y\}, k}^{\alpha+1, \sigma}$.

Proof: 1 . The case $\alpha<-1$ is obtained by straightforward estimations.

2. For $\alpha>-1$ we write

$$
I_{1}(f)\left(x, v^{A}, y\right)=\underbrace{\int_{0}^{y} f\left(s, v^{A}, y\right) d s}_{g_{1}}-\underbrace{\int_{0}^{x} f\left(s, v^{A}, y\right) d s}_{g_{2}} .
$$

We have $\partial_{v}^{\gamma} g_{1}=O\left(y^{\alpha+\sigma+1}\right), \partial_{x} g_{1}=0, y^{\ell} \partial_{y}^{\ell} \partial_{v}^{\gamma} g_{1}\left(x, v^{A}, y\right)=y^{\ell} \partial_{y}^{\ell-1}\left(\partial_{v}^{\gamma} f\left(y, v^{A}, y\right)\right)$, and all the estimates readily follow.

Lemma A.11 Let $\alpha, \sigma \in \mathbb{R}, k \in \mathbb{N} \cup\{\infty\}$,

1. If $f \in \mathscr{C}_{\{0 \leq x \leq y\}, k}^{\alpha, \sigma}, \sigma>-1$ then $I_{2}(f) \in \mathscr{C}_{\{0 \leq x \leq y\}, k}^{\alpha, \sigma+1}$.

2. If $f \in \mathscr{C}_{\{y=0\}, \infty}^{\sigma}$, then $I_{2}(f) \in \mathscr{F}_{\{0 \leq x \leq y\}, \infty}^{\sigma+1}$. 
Proof: 1 . One is tempted to argue from point 2. of Lemma A.11 by symmetry that $I_{2}(f) \in \mathscr{C}_{\{x=0\}, k}^{\alpha+\sigma+1}+\mathscr{C}_{\{0 \leq x \leq y\}, k}^{\alpha, \sigma+1}$, but this is not clear, because one is not allowed to integrate all the way to zero in $y$, as done in the proof of 2 . So we calculate directly:

$$
\begin{aligned}
\partial_{x}^{\ell} \partial_{v}^{\beta} \int_{x}^{y} f\left(x, v^{A}, s\right) d s & =\int_{x}^{y} \partial_{x}^{\ell} \partial_{v}^{\beta} f\left(x, v^{A}, s\right) d s-\left.\sum_{i=0}^{\ell-1} C_{\ell, i} \partial_{x}^{\ell-1-i} \partial_{y}^{i} \partial_{v}^{\beta} f\left(x, v^{A}, s\right)\right|_{s=x}, \\
\partial_{x}^{\ell} \partial_{y}^{i} \partial_{v}^{\beta} \int_{x}^{y} f\left(x, v^{A}, s\right) d s & =\partial_{x}^{\ell} \partial_{y}^{i-1} \partial_{v}^{\beta} f\left(x, v^{A}, y\right), \quad i \geq 1 .
\end{aligned}
$$

From (A.33) one immediately finds $\left|\partial_{x}^{\ell} \partial_{y}^{i} \partial_{v}^{\beta} I_{2}(f)\right| \leq C x^{\alpha-\ell} y^{\sigma+1-i}$ for $i \geq 1$. Since $\sigma>-1$, the first term in (A.32) is estimated by

$$
C x^{\alpha-\ell}\left|y^{\sigma+1}-x^{\sigma+1}\right| \leq C x^{\alpha-\ell} y^{\sigma+1},
$$

as desired. Similarly, each term in the sum is estimated by

$$
\left.C x^{\alpha-\ell+i+1} y^{\sigma-i}\right|_{y=x}=C x^{\alpha-\ell+1+\sigma} \leq C x^{\alpha-\ell} y^{\sigma+1}
$$

and the result follows.

2. The estimates in the $\ell=0$ case are proved similarly as in point 1 , see (A.32). For $1 \leq \ell \leq k$ and $1 \leq i+\ell+|\gamma| \leq k$ we have

$$
\left|\partial_{x}^{i} \partial_{y}^{\ell} \partial_{v}^{\gamma} I_{2}(f)\right|=\left|\partial_{x}^{i} \partial_{y}^{\ell-1} \partial_{v}^{\gamma} f\right|
$$

and the desired estimate is straightforward.

\section{A.6 Integral operators on $\mathscr{T}$ - and $\mathscr{F}$-spaces}

Proposition A.12 Let $\alpha>-1, \beta \geq k$. For any $\epsilon>0$ we have

$$
I_{1}\left(\mathscr{T}_{\{0 \leq x \leq y\}, \infty}^{\alpha,(\beta ; k)}\right) \subset y^{\epsilon} \stackrel{\stackrel{\mathscr{F}}{\alpha+1-\epsilon+\beta}}{\{0 \leq x \leq y\}, \infty}+\mathscr{T}_{\{0 \leq x \leq y\}, \infty}^{\alpha+1-\epsilon,(\beta ; k)} .
$$

REMARK A.13 We expect the result to remain valid with $\epsilon=0$, but the proof below fails for this value of $\epsilon$. In any case the current result is sufficient for our purposes.

ProOF: Write $\alpha+\beta=n-\sigma, n \in \mathbb{N}, \sigma \in[0,1)$. Let $f \in \mathscr{T}_{\{0 \leq x \leq y\}, \infty}^{\alpha,(\beta ; k)}$, set

$$
g(y, v)=\int_{0}^{y} f(s, y, v) d s
$$

We want to show that the function $g$ satisfies the hypotheses needed to construct the extensions of Section A.4. For $j$ such that $\alpha+\beta-j>-1$ we have

$$
\partial_{y}^{j} \partial_{v}^{\gamma} g(y, v)=\sum_{\ell+m=j-1} C(\ell, m) \partial_{x}^{\ell} \partial_{y}^{m} \partial_{v}^{\gamma} f(y, y, v)+\int_{0}^{y} \partial_{y}^{j} \partial_{v}^{\gamma} f(x, y, v) d x
$$


From the definition of $\mathscr{T}_{\{0 \leq x \leq y\}, \infty}^{\alpha,(\beta ; k)}$ one thus finds, for $0 \leq j \leq n$,

$$
\left|\partial_{y}^{j} \partial_{v}^{\gamma} g\right| \leq C y^{\alpha+\beta+1-j}|\ln y|^{N} .
$$

Next, we need to control the modulus of Hölder continuity of the top order derivatives. In order to do that, consider any of the terms appearing under the sum symbol in (A.34) with $j=n$. For $y^{\prime} \in B(y, y / 2)$ we write

$$
\begin{gathered}
\left|\partial_{x}^{\ell} \partial_{y}^{m} \partial_{v}^{\gamma}\left(f(y, y, v)-f\left(y^{\prime}, y^{\prime}, v\right)\right)\right|=|\int_{y}^{y^{\prime}} \underbrace{\left(\partial_{x}^{\ell+1} \partial_{y}^{m} \partial_{v}^{\gamma} f(s, s, v)+\partial_{x}^{\ell} \partial_{y}^{m+1} \partial_{v}^{\gamma} f(s, s, v)\right)}_{\leq C y^{-\sigma}|\ln y|^{N}} d s| \\
\leq C y^{-\sigma}\left|y^{\prime}-y\right||\ln y|^{N+1} \leq C^{\prime} y^{1-\sigma-\lambda}\left|y^{\prime}-y\right|^{\lambda}|\ln y|^{N+1} \text { (A.35) }
\end{gathered}
$$

for any $\lambda \in[0,1]$. Next,

$$
\begin{aligned}
\int_{0}^{y} & \partial_{y}^{n} \partial_{v}^{\gamma} f(x, y, v) d x-\int_{0}^{y^{\prime}} \partial_{y}^{n} \partial_{v}^{\gamma} f\left(x, y^{\prime}, v\right) d x \\
& =\int_{y^{\prime}}^{y} \partial_{y}^{n} \partial_{v}^{\gamma} f(x, y, v) d x+\int_{0}^{y^{\prime}}\left(\partial_{y}^{n} \partial_{v}^{\gamma} f(x, y, v)-\partial_{y}^{n} \partial_{v}^{\gamma} f\left(x, y^{\prime}, v\right)\right) d x
\end{aligned}
$$

The first term is estimated similarly to (A.35),

$$
\left|\int_{y^{\prime}}^{y} \partial_{y}^{n} \partial_{v}^{\gamma} f(x, y, v) d x\right| \leq C y^{1-\sigma-\lambda}\left|y^{\prime}-y\right|^{\lambda}|\ln y|^{N+1} .
$$

To control the second we will need a weighted interpolation inequality, obtained as follows: for $t \in[1 / 2,3 / 2]$ set

$$
\chi(t)=\partial_{y}^{n} \partial_{v}^{\gamma} f(x, t y, v) .
$$

Recall the inequality [14, Theorem A.5]

$$
\|\chi\|_{\lambda} \leq C_{\lambda}\|\chi\|_{1}^{\lambda}\|\chi\|_{0}^{1-\lambda}, \quad 0 \leq \lambda \leq 1,
$$

where $\|\cdot\|_{\mu}$ denotes the usual Hölder $C^{\mu}([1 / 2,3 / 2])$ norm. Applying (A.39) to $\chi$ defined in (A.38) gives

$$
\begin{aligned}
& \left|\partial_{y}^{n} \partial_{v}^{\gamma} f(x, y, v)-\partial_{y}^{n} \partial_{v}^{\gamma} f\left(x, y^{\prime}, v\right)\right| \\
& \quad \leq C(\underbrace{\frac{y}{x} x^{-\sigma}\left|\ln ^{N} x\right|}_{\leftrightarrow\|\chi\|_{1}})^{\lambda}(\underbrace{x^{-\sigma}\left|\ln ^{N} x\right|}_{\leftrightarrow\|\chi\|_{0}})^{1-\lambda} y^{-\lambda}\left|y-y^{\prime}\right|^{\lambda} \\
& \quad=C x^{-\lambda-\sigma\left|\ln ^{N} x \| y-y^{\prime}\right|^{\lambda} .}
\end{aligned}
$$

It follows that the last integral in (A.36) converges for $\lambda<1-\sigma$, leading to

$$
0 \leq \lambda+\sigma<1 \quad\left|\partial_{y}^{n} \partial_{v}^{\gamma} g(y, v)-\partial_{y}^{n} \partial_{v}^{\gamma} g\left(y^{\prime}, v\right)\right| \leq C y^{1-\sigma-\lambda}\left|y^{\prime}-y\right|^{\lambda}|\ln y|^{N} .
$$


Setting

$$
g_{0}=g, \quad g_{i}=0 \text { for } 1 \leq i \leq n,
$$

by Lemma A.8 there exists a function $h \in y^{1-\sigma-\lambda} \mathscr{F}_{\{0 \leq x \leq y\}, \infty}^{n+\lambda}$ for any $0 \leq$ $\lambda+\sigma<1$ such that $\left.\partial_{x}^{i} h\right|_{x=0}=g_{i}$. Define

$$
\hat{h}=I_{1}(f)-h .
$$

For $0 \leq i+j \leq n$ we write

$$
\begin{aligned}
\left|\partial_{x}^{i} \partial_{y}^{j} \partial_{v}^{\gamma} \hat{h}\right| & =\left|\partial_{x}^{i} \partial_{y}^{j} \partial_{v}^{\gamma}\left(\int_{x}^{y} f(s, \cdots) d s-h\right)\right| \\
& =\left|\partial_{x}^{i} \partial_{y}^{j} \partial_{v}^{\gamma}\left(\int_{0}^{y} f(s, \cdots) d s-h-\int_{0}^{x} f(s, \cdots) d s\right)\right| \\
& \leq \underbrace{\left|\partial_{x}^{i} \partial_{y}^{j}\left(\int_{0}^{y} \partial_{v}^{\gamma} f(s, \cdots) d s-\partial_{v}^{\gamma} h\right)\right|}_{I}+\underbrace{\left|\partial_{x}^{i} \int_{0}^{x} \partial_{y}^{j} \partial_{v}^{\gamma} f(s, \cdots) d s\right|}_{I I} .
\end{aligned}
$$

The estimate of $I I$ is straightforward:

$$
|I I| \leq C\left(x^{\alpha+1+\beta-i-j}+x^{\alpha+1-i} y^{\beta-j}+x^{\alpha+1+\beta-i-k} y^{k-j}\right)\left|\ln ^{N} x\right| .
$$

To estimate $I$ we use Taylor's formula,

$$
\begin{aligned}
\partial_{x}^{i} h(x, y, v) & =\sum_{\ell=0}^{n-i-j} \frac{x^{\ell}}{\ell !} \partial_{x}^{\ell+i} h(0, y, v)+\int_{0}^{x} \frac{(x-t)^{n-i-j}}{(n-i-j) !} \partial_{x}^{n+1-j} h(t, y, v) d t \\
& =\int_{0}^{x} \frac{(x-t)^{n-i-j}}{(n-i-j) !} \partial_{x}^{n+1-j} h(t, y, v) d t+ \begin{cases}g(y, v), & i=0 ; \\
0, & \text { otherwise. }\end{cases}
\end{aligned}
$$

This gives

$$
\begin{aligned}
|I| & =\int_{0}^{x} \frac{(x-t)^{n-i-j}}{(n-i-j) !} \underbrace{}_{\leq C y^{1-\sigma-\lambda} \mid \ln ^{N}} \underbrace{\left|\partial_{y}^{j} \partial_{x}^{n+1-j} \partial_{v}^{\gamma} h(t, y, v)\right|}_{t\left|\left(y^{n+\lambda-n-1}+t^{n+\lambda-n-1}\right) \leq 2 C y^{1-\sigma-\lambda} t^{\lambda-1}\right| \ln ^{N} t \mid} d t \\
& \leq C^{\prime} x^{n+\lambda-i-j} y^{1-\sigma-\lambda}\left|\ln ^{N+1} x\right| .
\end{aligned}
$$

This calculation also proves that

$$
h \in y^{1-\sigma-\lambda} \stackrel{\stackrel{\sim}{F}}{n+\lambda}\{0 \leq x \leq y\}, \infty .
$$

For $i+j>n$ we write

$$
\begin{aligned}
\left|\partial_{x}^{i} \partial_{y}^{j} \partial_{v}^{\gamma} \hat{h}\right| & =\left|\partial_{x}^{i} \partial_{y}^{j} \partial_{v}^{\gamma}\left(\int_{x}^{y} f(s, \cdots) d s-h\right)\right| \\
& \leq \underbrace{\left|\partial_{x}^{i} \partial_{y}^{j}\left(\int_{x}^{y} \partial_{v}^{\gamma} f(s, \cdots) d s\right)\right|}_{A}+\underbrace{\left|\partial_{x}^{i} \partial_{y}^{j} \partial_{v}^{\gamma} \hat{h}\right|}_{B} .
\end{aligned}
$$


The second term is immediately estimated by a constant times $x^{n+\lambda-i-j} y^{1-\sigma-\lambda}\left|\ln ^{N} x\right|$. The estimation of $A$ is simplest when $i>0$ as then we have

$$
|A|=\left|\partial_{x}^{i-1} \partial_{y}^{j} \partial_{v}^{\gamma} f(x, y, v)\right| \leq C\left(x^{\alpha+1+\beta-i-j}+x^{\alpha+1-i} y^{\beta-j}+x^{\alpha+1+\beta-i-k} y^{k-j}\right)\left|\ln ^{N} x\right| .
$$

When $i=0$ we use instead

$$
|A|=|\underbrace{\sum_{\ell+m=j-1} C(\ell, m) \partial_{x}^{\ell} \partial_{y}^{m} \partial_{v}^{\gamma} f(y, y, v)}_{\leq C y^{\alpha+1+\beta-j}\left|\ln ^{N} y\right| \leq C x^{\alpha+1+\beta-j}\left|\ln ^{N} x\right|}+\int_{x}^{y} \partial_{y}^{j} \partial_{v}^{\gamma} f(x, y, v)|,
$$

which is again estimated as in (A.44). Summarising, we have obtained

$$
\left|\partial_{x}^{i} \partial_{y}^{j} \partial_{v}^{\gamma} \hat{h}\right| \leq C\left(x^{\alpha+1+\beta-i-j}+x^{\alpha+1-i} y^{\beta-j}+x^{\alpha+1+\beta-i-k} y^{k-j}+x^{\alpha+1+\beta+\lambda+\sigma-i-j} y^{1-\sigma-\lambda}\right)\left|\ln ^{N} x\right|,
$$

for any $0 \leq \sigma+\lambda<1$. Setting $\lambda=1-\sigma-\epsilon$ and estimating $y^{\epsilon} \leq y_{0}^{\epsilon}, x^{\alpha} \leq y_{0}^{\epsilon} x^{\alpha-\epsilon}$, the result follows.

Proposition A.14 Let $\alpha+p \delta>-1$. For any $\epsilon>0$ we have

$$
\begin{aligned}
& \text { 1. } I_{1}\left(x^{p \delta} \ln ^{\ell} x \stackrel{\circ}{\mathscr{F}}_{\{0 \leq x \leq y\}, \infty}^{\alpha}\right) \subset y^{\epsilon} \stackrel{\circ}{\mathscr{F}}_{\{0 \leq x \leq y\}, \infty}^{\alpha+p \delta+1-\epsilon}+\mathscr{A}_{\{x=0\}, x \mathscr{F}_{\{0 \leq x \leq y\}, \infty}^{\alpha}}^{\alpha} . \\
& \text { 2. } I_{1}\left(\mathscr{A}_{\{x=0\}, x^{p \delta} \stackrel{\circ}{\mathscr{F}}_{\{0 \leq x \leq y\}, \infty}^{\alpha}}^{\delta}\right) \subset y^{\epsilon} \stackrel{\circ}{\mathscr{F}}_{\{0 \leq x \leq y\}, \infty}^{\alpha+p \delta+1-\epsilon}+\mathscr{A}_{\{x=0\}, x^{p \delta+1} \mathscr{\mathscr { F }}_{\{0 \leq x \leq y\}, \infty}^{\alpha}}^{\delta}
\end{aligned} .
$$

Proof: The proof of point 1. is essentially the same as that of Proposition A.12, once the integration by parts of (A.45) has been done; we outline the main steps for completeness. We write again $\alpha+p \delta=n-\sigma, n \in \mathbb{N}, \sigma \in[0,1)$.

Let $f \in \stackrel{\circ}{\mathscr{F}}_{\{0 \leq x \leq y\}, \infty}^{\alpha}$. First we integrate by parts, obtaining

$I_{1}\left(x^{p \delta} \ln ^{\ell} x f\right)=\left.\sum_{r=0}^{N} C(r) s^{p \delta+1} \ln ^{r} s f(s, y, v)\right|_{s=x} ^{s=y}-\sum_{r=0}^{N} \int_{x}^{y} C(r) s^{p \delta+1} \ln ^{r} s \partial_{x} f(s, y, v) d s$.

(The heuristics behind the integration by parts is that $\partial_{x} f$ behaves differently from $f$ at the boundary, in particular $\partial_{x} f$ vanishes at the boundary for $\alpha>1$, while $f$ itself does not.) We set

$$
g(y, v)=-\sum_{r=0}^{N} \int_{0}^{y} C(r) s^{p \delta+1} \ln ^{r} s \partial_{x} f(s, y, v) d s .
$$

A repetition of the argument in the proof of A.12 shows that the function $g$ satisfies the hypotheses needed to construct the extensions of Section A.4, with $m$ in Lemma A.8 equal to $n, \mu=1-\sigma$ and any $0 \leq \lambda<1-\sigma$. We set

$$
g_{0}=g, \quad g_{i}=0 \text { for } 1 \leq i \leq n .
$$


By Lemma A.8 there exists a function $h$, belonging to the space $y^{1-\sigma-\lambda} \mathscr{F}_{\{0 \leq x \leq y\}, \infty}^{n+\lambda}$ for any $0 \leq \lambda+\sigma<1$, such that $\left.\partial_{x}^{i} h\right|_{x=0}=g_{i}$. The Taylor expansion as in the previous proof proves further that

$$
h \in y^{1-\sigma-\lambda} \stackrel{\stackrel{\mathscr{F}}{n+\lambda}}{\{0 \leq x \leq y\}, \infty} .
$$

Let us now define

$$
\hat{h}=I_{1}\left(x^{p \delta} \ln ^{\ell} x f\right)-h-\left.\sum_{r=0}^{N} C(r) s^{p \delta+1} \ln ^{r} s f(s, y, v)\right|_{s=x} ^{s=y} .
$$

An argument as in the proof of Proposition A.12 gives $\hat{h} \in y^{\epsilon} \stackrel{\circ}{\mathscr{F}}_{\{0 \leq x \leq y\}, \infty}^{\alpha+p \delta+1-\epsilon}$.

What remains are the terms

$$
\left.\sum_{r=0}^{N} C(r) s^{p \delta+1} \ln ^{r} s f(s, y, v)\right|_{s=x} ^{s=y} .
$$

The part with $s=y$ is in $\stackrel{\circ}{\mathscr{F}}_{\{0 \leq x \leq y\}, \infty}^{\alpha+p \delta+1} \subset y^{\epsilon} \stackrel{\stackrel{\mathscr{F}}{\alpha}}{\alpha+p \delta+x \leq y\}, \infty}_{\{0 \leq x \leq 1, \infty}$ and the part with $s=x$ belongs to $\mathscr{A}_{\{x=0\}, x \mathscr{F}_{\{0 \leq x \leq y\}, \infty}^{\alpha}}^{\delta}$.

Point 2 follows immediately from point 1 using the expansion (A.8).

Proposition A.15 1. $I_{2}\left(\stackrel{\mathscr{F}}{\alpha}_{\{0 \leq x \leq y\}, \infty}^{\alpha}\right) \subset \stackrel{\circ}{\mathscr{F}}_{\{0 \leq x \leq y\}, \infty}^{\alpha}$.

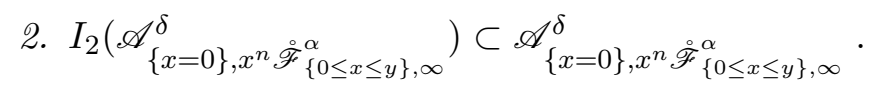

3. $I_{2}\left(\mathscr{T}_{\{0 \leq x \leq y\}, \infty}^{\alpha,(\beta ; k)}\right) \subset \mathscr{T}_{\{0 \leq x \leq y\}, \infty}^{\alpha,(\beta+1 ; k+1)}$.

Proof: 1. For $f \in \stackrel{\circ}{\mathscr{F}}_{\{0 \leq x \leq y\}, \infty}^{\alpha}$ define $F:=I_{2}(f)$. For $j \geq 1$ we have

$$
\partial_{x}^{i} \partial_{y}^{j} \partial_{v}^{\gamma} F(x, y, v)=\partial_{x}^{i} \partial_{y}^{j-1} \partial_{v}^{\gamma} f(x, y, v)
$$

and the estimates are obvious. For $j=0$ we use

$$
\left|\partial_{x}^{i} \partial_{v}^{\gamma} F\right| \leq \underbrace{\sum_{\ell+m=i-1} C(\ell, m)\left|\partial_{x}^{\ell} \partial_{y}^{m} \partial_{v}^{\gamma} f(x, x, v)\right|}_{\leq C x^{\alpha+1-i}\left|\ln ^{N} x\right|}+\underbrace{\int_{x}^{y}\left|\partial_{x}^{i} \partial_{v}^{\gamma} f(x, s, v)\right| d s}_{\leq C^{\prime} x^{\alpha-i} y\left|\ln ^{N} x\right|}
$$

In the first term we estimate $x^{\alpha+1-i} \leq x^{\alpha-i} y_{0}$, in the second $y \leq y_{0}$, and the proof is done.

2. The result follows from 1 and the definition of $\mathscr{A}_{\{x=0\}, F}^{\delta}$ (see Equation (A.8)).

3. For $f \in \mathscr{T}_{\{0 \leq x \leq y\}, \infty}^{\alpha,(\beta ; k)}$ let us define $F:=I_{2}(f)$. We have to estimate $\left|\partial_{x}^{i} \partial_{y}^{j} \partial_{v}^{\gamma} F\right|$. This is simplest for $j \geq 1$, we have then

$$
\left|\partial_{x}^{i} \partial_{y}^{j} \partial_{v}^{\gamma} F\right|=\left|\partial_{x}^{i} \partial_{y}^{j-1} \partial_{v}^{\gamma} f\right|
$$


and the appropriate estimates with $\beta \rightarrow \beta+1, k \rightarrow k+1$ follow from the definition of $\mathscr{T}_{\{0 \leq x \leq y\}, \infty}^{\alpha,(\beta ; k)}$. Again for $j=0$ we use

$$
\left|\partial_{x}^{i} \partial_{v}^{\gamma} F\right| \leq \underbrace{\sum_{\ell+m=i-1} C(\ell, m)\left|\partial_{x}^{\ell} \partial_{y}^{m} \partial_{v}^{\gamma} f(x, x, v)\right|}_{\leq C x^{\alpha+\beta+1-i}\left|\ln ^{N} x\right|}+\int_{x}^{y}\left|\partial_{x}^{i} \partial_{v}^{\gamma} f(x, s, v)\right| d s .
$$

The second term is estimated by

$$
C\left(x^{\alpha+\beta-i} y+x^{\alpha-i} y^{\beta+1}+x^{\alpha+\beta+1-i-k} y^{k+1}+x^{\alpha+\beta+1-i}\right)\left|\ln ^{N+1} x\right| .
$$

The calculation

$x^{\alpha+\beta-i} y\left|\ln ^{N+1} x\right|=x^{\alpha+(\beta+1)-(k+1)-i} x^{k} y\left|\ln ^{N+1} x\right| \leq x^{\alpha+(\beta+1)-(k+1)-i} y^{k+1}\left|\ln ^{N+1} x\right|$

ends the proof.

\section{B Polyhomogeneity of solutions for a class of lin- ear symmetric hyperbolic systems with smooth coef- ficients}

In this appendix we give a simple proof of a counterpart of [11, Theorem 3.4] without corner conditions. The $\mathscr{A}_{k}^{\delta}, \mathscr{C}_{l \mid 0}^{\alpha}$ and $\mathscr{C}^{\alpha}$ spaces are defined as in $[11$, Sections 3.3, 3.4 and (A.2)]. We work in the $\left(x, v^{A}, \tau\right)$ coordinates of [11], see Figure 1. Let us define the following space:

$$
\begin{aligned}
\widehat{\mathscr{F}}_{k}^{\alpha} & =\{f: \forall 0 \leq i+j+|\gamma| \leq k \\
& \left|\partial_{x}^{i} \partial_{\tau}^{j} \partial_{v}^{\gamma} f\right| \leq\left\{\begin{array}{ll}
C(x+2 \tau)^{\alpha-i-j} & \text { if } \alpha-i-j \geq 0 \\
C x^{\alpha-i-j} & \text { if } \alpha-i-j<0
\end{array}\right\} .
\end{aligned}
$$

This space is very similar, but not identical, to the space defined in (A.14), because of the log factor occurring in (A.14); that factor is not needed in the current section. Next, the space $\mathrm{A}_{k}^{\delta}$ is defined as the space of functions which can be written in the form

$$
\sum_{i=0}^{k} \sum_{j=0}^{N_{i}} f_{i j}(x+2 \tau)^{i \delta} \ln ^{j}(x+2 \tau)+f_{k \delta+\epsilon},
$$

for some $\epsilon>0$, with functions $f_{i j} \in C_{\infty}(\bar{\Omega})$ and $f_{k \delta+\epsilon} \in \widehat{\mathscr{F}}_{\infty}^{k \delta+\epsilon}$. We will always assume $1 / \delta \in \mathbb{N}$.

We note the following properties:

Proposition B.1 1. $\widehat{\mathscr{F}}_{k}^{\alpha} \subset \widehat{\mathscr{F}}_{k}^{\beta}$ for $\alpha \geq \beta$, 
2. $\mathscr{C}_{l \mid 0}^{\alpha} \subset \widehat{\mathscr{F}}_{l}^{\alpha}$,

3. set $\hat{f}\left(x, v^{A}, \tau\right):=f\left(x+2 \tau, v^{A}\right)$, then for $\alpha \notin \mathbb{Z}$ we have $\hat{f} \in \widehat{\mathscr{F}}_{\infty}^{\alpha}$ for $f \in \mathscr{C}_{\infty}^{\alpha}(\{\tau=0\})$ and $\hat{f} \in \mathrm{A}_{k}^{\delta}$ for $f \in \mathscr{A}_{k}^{\delta}(\{\tau=0\})$,

4. $f \in \widehat{\mathscr{F}}_{\infty}^{\alpha} \Rightarrow x f \in \widehat{\mathscr{F}}_{\infty}^{\alpha+1}$,

5. $f \in \widehat{\mathscr{F}}_{\infty}^{\alpha} \Rightarrow \partial_{x} f, \partial_{\tau} f \in \widehat{\mathscr{F}}_{\infty}^{\alpha-1}, \partial_{v} f \in \widehat{\mathscr{F}}_{\infty}^{\alpha}$.

Proof: 1: This follows from the definition of $\widehat{\mathscr{F}}_{\infty}^{\alpha}$ and from the fact that $(x+2 \tau)^{\alpha-\beta} \leq C$.

2: $f \in \bar{C}_{l \mid 0}^{\alpha}$ iff for all $m, n, \gamma$ satisfying $0 \leq m+n+|\gamma| \leq l$ we have $\left|\partial_{x}^{m} \partial_{\tau}^{n} \partial_{v}^{\gamma} f\right| \leq C x^{\alpha-m-n}$. For $\alpha-m-n<0$ this is exactly the estimate we need and for $\alpha-m-n \geq 0$ we have $x^{\alpha-m-n} \leq(x+2 \tau)^{\alpha-m-n}$.

3: Since $f \in \mathscr{C}^{\alpha}(\tau=0)$ we have $\left|\partial_{x}^{m} \partial_{v}^{\gamma} f\left(x, v^{A}\right)\right| \leq C x^{\alpha-m}$. The following holds: $\left|\partial_{x}^{m} \partial_{\tau}^{n} \partial_{v}^{\gamma} \hat{f}\left(x, v^{A}, \tau\right)\right|=2^{n}\left|\partial_{x}^{m+n} \partial_{v}^{\gamma} f\left(x+2 \tau, v^{A}\right)\right| \leq C(x+2 \tau)^{\alpha-m-n}$, which together with $(x+2 \tau)^{\beta} \leq x^{\beta}$ for $\beta<0$ yields the result. The statement concerning $\mathscr{A}$ follows from the application of the result to the error term $f_{k \delta+\epsilon} \in$ $\mathscr{C}_{\infty}^{k \delta+\epsilon}$

4: This follows from $\partial_{x}^{m} \partial_{\tau}^{n} \partial_{v}^{\gamma}(x f)=m \partial_{x}^{m-1} \partial_{\tau}^{n} \partial_{v}^{\gamma} f+x \partial_{x}^{m} \partial_{\tau}^{n} \partial_{v}^{\gamma} f$.

5: Obvious.

REMARK B.2 Let $\gamma \in \mathbb{R}$ and $f \in \widehat{\mathscr{F}}_{\infty}^{\alpha}$. It may happen that $x^{\gamma} f \notin \widehat{\mathscr{F}}_{\infty}^{\alpha+\gamma}$ or $(x+2 \tau)^{\gamma} f \notin \widehat{\mathscr{F}}_{\infty}^{\alpha+\gamma}$.

The behavior of $\widehat{\mathscr{F}}^{\alpha}$ spaces under integration is summarised in the following proposition:

Proposition B.3 Let $f \in \widehat{\mathscr{F}}_{\infty}^{\alpha}, \alpha \in \mathbb{R} \backslash \mathbb{Z}$. Then

$$
\begin{gathered}
I_{1}(f):=\int_{x}^{x+2 \tau} f\left(s, v^{A}, \frac{1}{2} x+\tau-\frac{1}{2} s\right) d s \in \widehat{\mathscr{F}}_{\infty}^{\alpha+1}, \\
I_{2}(f):=\int_{0}^{\tau} f\left(x, v^{A}, s\right) d s \in \widehat{\mathscr{F}}_{\infty}^{\alpha} .
\end{gathered}
$$

Proof: First we note that

$$
\partial_{x} I_{1}(f)=I_{1}\left(\partial_{x} f\right), \quad \partial_{\tau} I_{1}(f)=f\left(x+2 \tau, v^{A}, 0\right)+I_{1}\left(\partial_{\tau} f\right),
$$

and for the latter integral

$$
\partial_{x} I_{2}(f)=I_{2}\left(\partial_{x} f\right), \quad \partial_{\tau} I_{2}(f)=f\left(x, v^{A}, \tau\right) .
$$

Then the result is easily obtained, with the following formula being the key estimate:

$$
I_{1}\left(x^{\alpha}\right)=\frac{(x+2 \tau)^{\alpha+1}}{\alpha+1}-\frac{x^{\alpha+1}}{\alpha+1} \leq\left\{\begin{array}{ll}
C(x+2 \tau)^{\alpha+1} & \alpha+1>0 \\
C x^{\alpha+1} & \alpha+1<0
\end{array} .\right.
$$


It is obvious that the standard space of polyhomogeneous functions $\mathscr{A}_{k}^{\delta}$ is closed with respect to $x \partial_{x}$, the same is true for $x^{\beta} \mathscr{A}_{k}^{\delta}$. The $\mathrm{A}_{k}^{\delta}$ space does not have this property (terms like $\frac{x}{x+2 \tau}$ may appear). In order to fix this we introduce another space, denoted by $\mathrm{A}_{k \pm}^{\delta}$, consisting of functions which can be written as a finite sum of terms of the form

$$
f_{i j \ell} x^{i}(x+2 \tau)^{j \delta} \ln ^{\ell}(x+2 \tau)
$$

with $i, \ell \in \mathbb{N}, j \in \mathbb{Z}, 0 \leq i+j \delta \leq k \delta$, plus an error term $f_{k \delta+\epsilon} \in \widehat{\mathscr{F}}_{\infty}^{k \delta+\epsilon}$. The space $\mathrm{A}_{k \pm}^{\delta}$ as well as $(x+2 \tau)^{\beta} \mathrm{A}_{k \pm}^{\delta}$ is closed with respect to $x \partial_{x}, x \partial_{\tau}$ and $\partial_{v}$.

We use the symbols $\mathscr{A}_{k}^{\delta}, \AA_{k}^{\delta}$ and $\AA_{k \pm}^{\delta}$ for spaces of function which can be written as sums of a finite number of terms as above without the error term.

If we restrict ourselves to one value of $\tau \geq 0$ then there is a correspondence between the spaces $\mathscr{A}_{k}^{\delta}$ and $\mathrm{A}_{k \pm}^{\delta}$. Obviously any function belonging to $\mathscr{A}_{k}^{\delta}$ belongs also to $\mathscr{\mathscr { A }}_{k}^{\delta}+\mathrm{A}_{k}^{\delta}$ and $\dot{\mathscr{A}}_{k}^{\delta}+\mathrm{A}_{k \pm}^{\delta}$. The relation in the opposite direction is given by the following Lemma:

Lemma B.4 Let $\stackrel{\circ}{\tau} \geq 0$ and let $\left.f \in\left(\mathscr{A}_{k}^{\delta}+\mathrm{A}_{k \pm}^{\delta}\right)\right|_{\tau=\tau}$. Then $f \in \mathscr{A}_{k}^{\delta}$.

Proof: The "error term' $r \in \widehat{\mathscr{F}}_{\infty}^{k \delta+\epsilon}$ in $f$ equals

$$
f-\sum_{i, j, \ell} f_{i j \ell} x^{i}\left(x+2 \stackrel{\circ}{\tau}^{j \delta} \ln ^{\ell}(x+2 \stackrel{\circ}{\tau})-\sum_{i, j} f_{i j} x^{i \delta} \ln ^{j} x,\right.
$$

with both sums being finite. From the definition of our spaces we have

$$
\left|\partial_{x}^{n} \partial_{v}^{\alpha} r\right| \leq C \cdot \begin{cases}(x+2 \stackrel{\circ}{\tau})^{k \delta+\epsilon-n} & \text { if } k \delta+\epsilon-n \geq 0 \\ x^{k \delta+\epsilon-n} & \text { if } k \delta+\epsilon-n<0 .\end{cases}
$$

We want to show that there exist $\hat{f}_{i j}$ such that

$$
\left|\partial_{x}^{n} \partial_{v}^{\alpha}\left(f-\sum_{i j} \hat{f}_{i j} x^{i \delta} \ln ^{j} x\right)\right| \leq C \cdot x^{k \delta+\epsilon-n}
$$

The estimate for $k \delta+\epsilon-n<0$ is trivial so we focus on the first case. Let us notice that $k \delta+\epsilon=m+\alpha$ with $m=\lfloor k \delta+\epsilon\rfloor$ and $\alpha \in[0,1)$. Now, $r$ has $m x$-derivatives $\partial_{x}^{n} \partial_{v}^{\alpha} r$ bounded by a constant and the $(m+1)$-th $x$-derivative $\partial_{x}^{m+1} \partial_{v}^{\alpha} r$ is bounded by $C \cdot x^{\alpha-1}$. Therefore we can Taylor-expand $r$ up to the order $m$. The rest is of order $m+\alpha$ :

$$
\left|r(x, v)-\sum_{i=0}^{m} \frac{\partial_{x}^{i} r(x=0, v)}{i !} x^{i}\right| \leq C \cdot x^{m+\alpha}
$$

and the proof is easily completed.

The following proposition gives the behaviour of polyhomogeneous spaces under integration: 
Proposition B.5 1. Let $f \in(x+2 \tau)^{\beta} \AA_{k \pm}^{\delta}$. There exists $f_{\beta+k \delta+\epsilon} \in \widehat{\mathscr{F}}_{\infty}^{\beta+k \delta+\epsilon}$, for some $\epsilon>0$, such that

$$
\begin{gathered}
I_{2}(f) \in(x+2 \tau)^{\beta} \AA_{k \pm}^{\delta}+x^{\beta} \dot{\mathscr{A}}_{k}^{\delta}+f_{\beta+k \delta+\epsilon} ;, \\
I_{1}(f) \in(x+2 \tau)^{\beta} \AA_{k \pm}^{\delta}+f_{\beta+k \delta+\epsilon} .
\end{gathered}
$$

2. Let $f \in x^{\beta} \mathscr{\mathscr { A }}_{k}^{\delta}$. There exists $f_{\beta+k \delta+\epsilon} \in \widehat{\mathscr{F}}_{\infty}^{\beta+k \delta+\epsilon}$ such that

$$
I_{1}(f) \in(x+2 \tau)^{\beta} \AA_{k \pm}^{\delta}+x^{\beta} \mathscr{A}_{k}^{\delta}+f_{\beta+k \delta+\epsilon} .
$$

Proof: The proposition readily follows from the next two lemmata.

Lemma B.6 Let $f$ be a smooth function, $p \in \mathbb{R}, k \in \mathbb{N}$. For all $\alpha \in \mathbb{R}$ there exists $N \in \mathbb{N}$, sequences of numbers $A_{i} \in p+1+\mathbb{N}, B_{i} \in \mathbb{N}$, a sequence of smooth functions $f_{i}$ and a function $f_{\alpha} \in \widehat{\mathscr{F}}_{\infty}^{\alpha}$ such that

$$
I_{1}\left(f x^{p} \ln ^{k} x\right)=\sum_{i=1}^{N} f_{i}\left((x+2 \tau)^{A_{i}} \ln ^{B_{i}}(x+2 \tau)-x^{A_{i}} \ln ^{B_{i}} x\right)+f_{\alpha} .
$$

PROOF: Integration by parts yields the result for $f=1$. The result for general $f$ is also obtained by integration by parts:

$$
\int f v^{\prime}=f v-\int f^{\prime} v
$$

where $v^{\prime}=s^{p} \ln ^{k} s$. Using the result for $f=1$ one gets that $v$ has a power of $s$ one higher than $v^{\prime}$. Repeating this integration a finite number of times yields a result with an error term in $\widehat{\mathscr{F}}_{\infty}^{n}$ and $n$ high enough.

Lemma B.7 Let $f$ be smooth, $i \geq-1$ and $\alpha>0$. Then there exists $n \in \mathbb{N}$, a sequence of smooth functions $f_{j}$ and $f_{\alpha} \in \widehat{\mathscr{F}}_{\infty}^{\alpha}$ such that

$$
I_{1}\left(f x^{i}\right)=\sum_{j=0}^{n} f_{j}\left((x+2 \tau)^{i+j+1}-x^{i+j+1}\right)+f_{\alpha} .
$$

Proof: Since $f$ is smooth it can be expanded in powers of $x$ to any order and the result follows.

Proposition B.8 Let $\varphi$ be a solution of

$$
\partial_{\tau} \varphi+b \varphi=c
$$

with

$$
b \in C_{\infty}(\bar{\Omega})
$$

Let us assume that

$$
\varphi(x, v, 0) \in x^{\beta} \mathscr{A}_{k}^{\delta}
$$

and

$$
c \in(x+2 \tau)^{\beta} \AA_{k \pm}^{\delta}+x^{\beta} \mathscr{\mathscr { A }}_{k}^{\delta}+\widehat{\mathscr{F}}_{\infty}^{\alpha}+C_{\infty}(\bar{\Omega}) .
$$

Then

$$
\varphi \in(x+2 \tau)^{\beta} \AA_{k \pm}^{\delta}+x^{\beta} \dot{\mathscr{A}}_{k}^{\delta}+\widehat{\mathscr{F}}_{\infty}^{\min (\alpha, \beta+k \delta+1)}+C_{\infty}(\bar{\Omega}) .
$$


Proof: The solution of the equation at hand may be expressed as

$$
\varphi(\cdot, \tau)=R(\cdot, \tau) \varphi(\cdot, 0)+\int_{0}^{\tau} R(\cdot, s) c(\cdot, s) d s
$$

where $R(x, v, \tau)$ is the resolvent of the equation $\partial_{\tau} \varphi=-b \varphi$. It is a standard result that for $b \in C_{\infty}(\bar{\Omega})$ we have also $R \in C_{\infty}(\bar{\Omega})$. Then the result follows from the analysis of the above formula and Propositions B.3 and B.5.

Now we are ready to pass to the proof of polyhomogeneity of solutions:

Theorem B.9 Let $\alpha, \beta \in \mathbb{R}, k \in \mathbb{N}$, and let $(\varphi, \psi) \in \mathscr{C}_{\infty}^{\alpha}$ be a solution of

$$
\begin{aligned}
\partial_{\tau} \varphi+B_{11} \varphi+B_{12} \psi & =L_{11} \varphi+L_{12} \psi+a \\
e_{+} \psi+B_{21} \varphi+B_{22} \psi & =L_{21} \varphi+L_{22} \psi+b
\end{aligned}
$$

where $e_{+}=\partial_{\tau}-2 \partial_{x}$. Suppose that

$$
L_{i j}=L_{i j}^{A} \partial_{A}+x L_{i j}^{\tau} \partial_{\tau}+x L_{i j}^{x} \partial_{x}
$$

with

$$
L_{11}^{\mu} \in x C_{\infty}(\bar{\Omega}), \quad L_{21}^{\mu}, L_{12}^{\mu}, L_{22}^{\mu} \in C_{\infty}(\bar{\Omega})
$$

and that

$$
\begin{gathered}
B_{a b} \in C_{\infty}(\bar{\Omega}), \\
a, b \in C_{\infty}(\bar{\Omega}), \quad \varphi(0), \psi(0) \in x^{\beta} \mathscr{A}_{k}^{\delta}\left(M_{x_{0}}\right) .
\end{gathered}
$$

Then

$$
\varphi, \psi \in x^{\beta} \mathscr{\mathscr { A }}_{k}^{\delta}+(x+2 \tau)^{\beta} \stackrel{\AA}{k \pm}_{k \pm}^{\delta}+C_{\infty}(\bar{\Omega})+\widehat{\mathscr{F}}_{\infty}^{\beta+k \delta+\epsilon}
$$

for some $\epsilon>0$.

Proof: The proof is very similar to the proof of Theorem [11, Theorem 3.4]. First we notice that

$$
(\varphi, \psi) \in \widehat{\mathscr{F}}_{\infty}^{\alpha},
$$

which is due to Proposition B.1 point 2. For the purpose of the proof it will be convenient to use the following notation:

$$
\mathscr{S}^{\alpha}:=\widehat{\mathscr{F}}_{\infty}^{\alpha}+C_{\infty}+x^{\beta} \mathscr{\mathscr { A }}_{k}^{\delta}+(x+2 \tau)^{\beta} \AA_{k \pm}^{\delta} .
$$

To prove the theorem we need to show

$$
(\varphi, \psi) \in \mathscr{S}^{\beta+k \delta+\epsilon}
$$

We rewrite the equations at hand as

$$
\begin{aligned}
\partial_{\tau} \varphi+B_{11} \varphi & =c_{1}, \\
e_{+} \psi & =c_{2},
\end{aligned}
$$


where

$$
\begin{aligned}
& c_{1}:=L_{11} \varphi+L_{12} \psi+a-B_{12} \psi, \\
& c_{2}:=L_{21} \varphi+L_{22} \psi+b-B_{21} \varphi-B_{22} \psi .
\end{aligned}
$$

Let us start with the second of the equations. The integration yields

$$
\psi\left(x, v^{A}, \tau\right)=\psi\left(x+2 \tau, v^{A}, 0\right)+\frac{1}{2} I_{1}\left(c_{2}\right) .
$$

We have

$$
c_{2} \in \widehat{\mathscr{F}}_{\infty}^{\alpha}+C_{\infty} \subset \mathscr{S}^{\alpha}
$$

Propositions B.3 and B.5 together with B.1 point 1 yield

$$
I_{1}\left(c_{2}\right) \in \mathscr{S}^{\min (\alpha+1, \beta+k \delta+\epsilon)} .
$$

From Proposition B.1 point 3 we have

$$
\psi\left(x+2 \tau, v^{A}, 0\right) \in \mathscr{S}^{\beta+k \delta+\epsilon} .
$$

Therefore

$$
\psi \in \mathscr{S}^{\min (\alpha+1, \beta+k \delta+\epsilon)} .
$$

Now we estimate $c_{1}$ to be in $\mathscr{S}^{\min (\alpha+1, \beta+k \delta+\epsilon)}$ and use Proposition B. 8 to get

$$
\varphi \in \mathscr{S}^{\min (\alpha+1, \beta+k \delta+\epsilon)} .
$$

Then we repeat this procedure, with $c_{2}$ now in $\mathscr{S}^{\min (\alpha+1, \beta+k \delta+\epsilon)}$ and get first $\psi$ then $\varphi$ in the appropriate $\mathscr{S}$ space with the error-term index increased by one. After a finite number of steps we get (B.7).

REMARK B.10 If we make an additional assumption that $L_{i j}^{\tau}=L_{i j}^{x}=0$, we obtaint the following variant of the result:

$$
\varphi, \psi \in x^{\beta} \dot{\mathscr{A}}_{k}^{\delta}+(x+2 \tau)^{\beta} \stackrel{\circ}{k}_{k}^{\delta}+C_{\infty}(\bar{\Omega})+\widehat{\mathscr{F}}_{\infty}^{\beta+k \delta+\epsilon} .
$$

The $\AA_{k \pm}^{\delta}$ space has been replaced by $\AA_{k}^{\delta}$.

\section{References}

[1] M.T. Anderson and P.T. Chruściel, Asymptotically simple solutions of the vacuum Einstein equations in even dimensions, Commun. Math. Phys. (2005), 557-577, gr-qc/0412020.

[2] L. Andersson and P.T. Chruściel, On asymptotic behavior of solutions of the constraint equations in general relativity with "hyperboloidal boundary conditions", Dissert. Math. 355 (1996), 1-100.

[3] Y. Choquet-Bruhat, Global existence theorems by the conformal method, Recent developments in hyperbolic equations (Pisa, 1987), Longman Sci. Tech., Harlow, 1988, pp. 16-37. 
[4] Global solutions of Yang-Mills equations on anti-de Sitter spacetime, Classical Quantum Gravity 6 (1989), 1781-1789.

[5] _ Global existence of wave maps, Rend. Circ. Mat. Palermo (2) Suppl. (1998), 143-152, Proceedings of the IX International Conference on Waves and Stability in Continuous Media (Bari, 1997).

[6] Y. Choquet-Bruhat and Chao Hao Gu, Existence globale d'applications harmoniques sur l'espace-temps de Minkowski $M_{3}$, C. R. Acad. Sci. Paris Sér. I Math. 308 (1989), 167-170.

[7] Y. Choquet-Bruhat and N. Noutchegueme, Solutions globales du système de Yang-Mills-Vlasov (masse nulle), C. R. Acad. Sci. Paris Sér. I Math. 311 (1990), 785-788.

[8] D. Christodoulou, Global solutions of nonlinear hyperbolic equations for small initial data, Comm. Pure Appl. Math. 39 (1986), 267-282. MR $87 \mathrm{c}: 35111$

[9] P.T. Chruściel and E. Delay, On mapping properties of the general relativistic constraints operator in weighted function spaces, with applications, Mém. Soc. Math. de France. 94 (2003), 1-103, gr-qc/0301073v2.

[10] P.T. Chruściel, J. Jezierski, and J. Kijowski, Hamiltonian field theory in the radiating regime, Lect. Notes in Physics, vol. m70, Springer, Berlin, Heidelberg, New York, 2001, URL http://www.phys.univ-tours.fr/ piotr/ papers/hamiltonian_structure.

[11] P.T. Chruściel and O. Lengard, Solutions of wave equations in the radiating regime, Bull. Soc. Math. de France 133 (2003), 1-72, math.AP/0202015.

[12] P.T. Chruściel, M.A.H. MacCallum, and D. Singleton, Gravitational waves in general relativity. XIV: Bondi expansions and the "polyhomogeneity" of Scri, Phil. Trans. Roy. Soc. London A 350 (1995), 113-141.

[13] H. Friedrich, Einstein's equation and geometric asymptotics, Gravitation and Relativity: At the turn of the Millenium (Pune) (N. Dadhich and J. Narlikar, eds.), IUCAA, 1998, Proceedings of GR15, pp. 153-176.

[14] L. Hörmander, The boundary problems of physical geodesy, Arch. Rat. Mech. Analysis 62 (1976), 1-52.

[15] O. Lengard, Solutions of the Einstein's equation, wave maps, and semilinear waves in the radiation regime, Ph.D. thesis, Université de Tours, 2001, http://www/phys.univ-tours.fr/〜piotr/papers/batz.

[16] V. Moncrief and J. Isenberg, Symmetries of cosmological Cauchy horizons, Commun. Math. Phys. 89 (1983), 387-413.

[17] L.A. Tamburino and J. Winicour, Gravitational fields in finite and conformal Bondi frames, Phys. Rev. 150 (1966), 1039-1053. 